\title{
Mesoscale Variability and Mean Flow Interaction Near the Gulf Stream as Seen by Satellite Altimetry and Numerical Modelling
}

by

\section{Juan Botella}

M.Sc., Centro de Investigación Cientifica y Educación Superior de Ensenada, México. 1996 B.Sc., Universidad Autónoma de Baja California, México. 1993

Submitted to the Joint Program in Physical Oceanography in partial fulfillment of the requirements for the degree of

Master of Science

MBL/WHOI

at the

MASSACHUSETTS INSTITUTE OF TECHNOLOGY and

LIBRARY

Woods Hole, MA

Marine Biological Laboratory

Woods Hole Oceanographic Institution

WOODS HOLE OCEANOGRAPHIC INSTITUTION

September 2001

(C)Juan Botella, MMI. All rights reserved.

The author hereby grants to MIT and WHOI permision to reproduce and distribute publicly paper and electronic copies of the thesis document in whole or in part. 


\title{
Mesoscale Variability and Mean Flow Interaction Near the Gulf Stream as Seen by Satellite Altimetry and Numerical Modelling
}

by

Juan Botella

\author{
Submitted to the Joint Program in Physical Oceanography \\ on August 18, 2001, in partial fulfillment of the \\ requirements for the degree of \\ Master of Science
}

\begin{abstract}
The interaction between the eddy field and the mean flow near the Gulf Stream is studied here using satellite altimeter measurements and an eddy resolving numerical model. The eddy vorticity flux in the quasigeostrophic framework is obtained from the stream function standard deviation and spatial correlation function assuming the correlation function is homogeneous. An analytical expression is found for the stream function correlation using the altimetric and numerical data. Cases when the correlation function is anisotropic are compared to the isotropic case previously studied by Hogg (1993), who found that the eddy vorticity flux drives two counter rotating gyres on either side of the stream. The anisotropy can be important in the eddy vorticity flux, even when its departure from the isotropic case is small. Meridional or zonal anisotropies can drive recirculation gyres similar in strength and position to the ones driven by the isotropic case. The results when including anisotropy in the diagonal direction suggest that the homogenoeus assumption may not be valid.
\end{abstract}

Thesis Supervisor: Nelson G. Hogg

Title: Senior Scientist. Woods Hole Oceanographic Institution. 


\section{Acknowledgments}

My time in the Joint Program has been a wonderful adventure. Challenges have risen over the horizon; some I have conquered, others conquered me, and a few more I surrended to. Even those to which I yielded have proven to be useful lessons, and only a small number I sorted alone. Diversity is what comes to my mind when I think in those who I need to thank. A diverse group of people that made my days in WHOI, MIT and their surroundings, days to be remembered with pleasure.

Thanks to Nelson, a great mentor, for his reliable advice and never ending patience; thanks to Carl Wunsch for his support during my journey, and thanks to Nelson, Carl, Breck Owens and Mike Spall who helped me take a tough decision at the right time. Thanks to Steve Jayne and Frank Bryan for providing the numerical simulation. My gratitude to Charmaine King for her assistance with the TOPEX/Poseidon data, to Karen Polensky for her editorial advice, and Galen McKinley for also reviewing the manuscript, helping with the bibliography and a couple of figures, and for having patience through the orbit error times. Thanks to the education office in WHOI for all the support, with special mention to Julita, gracias. Lisa, Linda, Bud and Helen in MIT, thanks.

More than a degree I have gained friends and acquaintances; it is not me who will differentiate between them, but time and distance: Valerie, Pablo, Paulo, Turi, Nelson, Carl, Juli, Adrian, Fernanda, Didi, Albert, Francois, Jake, Avon, Judith, Richard, Jelena, Carl Reid, Patrick, Daniel, Kerim, Xiaoyun, Arnaud, Fabio, Bob and Jan. Thank you for sharing your world with me.

Special people I thank apart, breaking with equality: Alex, Doinita \& Anatole, grand famille, Je apprend tout plein des ils (but not French), a mulţumi. Mercy pour Christophe et sa éternel sourient. To Galen, my partner, bestfriend and lover. Suerte de haberte encontrado, te amo. Gracias a mis padres y hermanos por su amor comprensión y apoyo. Y gracias a Momo.

This work was founded in part by a Fulbright/CONACyT fellowship and a J. Seward Johnson Fellowship through the Education Office in WHOI. Gracias. 


\section{Contents}

1 Introduction $\quad 15$

1.1 Eddy Field- Mean Flow Interaction . . . . . . . . . . . . . 15

2 Satellite Altimetry and Numerical Model 21

2.1 Satellite Altimetry. . . . . . . . . . . . . . . . 21

2.1.1 Errors and Corrections. . . . . . . . . . . 22

2.2 Numerical Model . . . . . . . . . . . . . . . . 26

3 TOPEX/Poseidon 29

3.1 Data Processing. . . . . . . . . . . . . . . . . . 29

3.2 Eddy Kinetic Energy and Ellipse Variances . . . . . . . . . . . . 31

3.3 Alongtrack Correlations . . . . . . . . . . . . . . 33

3.3 .1 Barotropic Signals . . . . . . . . . . . . 37

3.3.2 Orbit Error Correction. . . . . . . . . . . . . . . 43

3.4 Two-dimension Correlation . . . . . . . . . . . . . . 43

3.5 Chapter Summary . . . . . . . . . . . . . 57

4 Numerical Model $\quad 61$

4.1 Velocity Correlations . . . . . . . . . . . . . 63

4.2 Along Track Correlations . . . . . . . . . . . . . . . 65

4.3 Two Dimensional Correlations . . . . . . . . . . . . . 66

4.4 Chapter Summary . . . . . . . . . . . . . . 78 
5 Eddy Field Mean Flow Interaction $\quad 79$

5.1 Equations of Motion . . . . . . . . . . . . . . . . 79

5.1.1 Quasigeostrophic Approximation . . . . . . . . . . . 81

5.1.2 Eddy vorticity Flux in Terms of the Stream Function Correlation. 85

5.2 Vorticity Balance for the Gulf Stream Region . . . . . . . . . 90

5.2.1 Anisotropy Oriented Zonally or Meridionally . . . . . . . . . 92

5.2 .2 Diagonal Anisotropy. . . . . . . . . . . . . . . . . 96

5.3 Chapter Summary . . . . . . . . . . . . . . . . . . . . 98

$\begin{array}{lll}6 & \text { Discussion and Conclusion } & 101\end{array}$

$\begin{array}{ll}\text { A Orbit Error. } & 105\end{array}$ 


\section{List of Figures}

1-1 Root mean square for TOPEX/Poseidon's sea surface height in $\mathrm{cm}$ (repeat cycles 8 to 112). . . . . . . . . . . . . . . . 17

1-2 Contours of $\overline{u^{\prime} v^{\prime}}$ from a barotropic quasigeostrophic model. A zonal jet is imosed as boundary condition on the East and West boundaries. Contour interval is $0.001 \mathrm{~m}^{2} s_{2}$. Solid lines are positive and dashed lines are negative. From Jayne et al. (1996) _ . . . . . . . . . 18

2-1 Schematic summary of the corrections that must be applied to the altimeter range measurement $R$. escanear figura 1 de $F u \&$ Cazenave

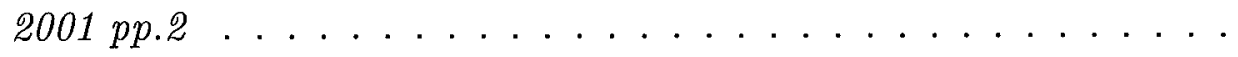

3-1 TOPEX/Poseidon ground tracks in the Gulf Stream area . . . . . . 30

3-2 Eddy kinetic energy computed from the geostrophic velocity at the satellite's cross overs . . . . . . . . . . . . . . . . . . 32

3-3 Variance ellipses from TOPEX/Poseidon data, normalized with maximum semimayor axis $\ldots \ldots \ldots \ldots \ldots$

3-4 scheme of the directions for the alongtrack correlations. . . . . . . 36 
3-5 Along track correlation at cross overs formed by arcs 74 and 89 ($\left.51^{\circ} \mathrm{lon}, 37.1^{\circ} \mathrm{lat}\right)$, (a) and (b), and by transects 176 and 191 (-56.7 lon, $37.1^{\circ}$ lat), (c) and (d). The directions are SE (solid line) and NW (broken line) for even numbered arcs, (a) and (c), and NE (solid line) and SW (broken lines) for odd numbered arcs, (b) and (d). The dotted line is the smallest positive indistinguishable form zero . . . . . . .

3-6 Correlation along (a) $24 \mathrm{SE}$, (b) $74 \mathrm{NW}$, (c) $100 \mathrm{NW}$, and (d) $76 \mathrm{NW}$. They correspond to the crossovers $24 / 13\left(-58.1^{\circ}\right.$ lon $29.7^{\circ}$ lat), $74 / 139$ ($\left.45.3^{\circ} \mathrm{lon}, 26.8^{\circ} \mathrm{lat}\right), 100 / 165\left(-53.8^{\circ} \mathrm{lon}, 26.8^{\circ} \mathrm{lat}\right)$, and $176 / 241\left(-51^{\circ} \mathrm{lon}\right.$, $26.8^{\circ}$ lat) at panel d) . . . . . . . . . . . .

3-7 Along-track correlation at cross over made by arcs 74 and 165, after the removal of the barotropic signal from a numerical model (solid line), as proposed by Stammer and Wunsch (2000), and without the correction (broken line). The dotted line corresponds to the lowest value that is significantly different from zero $\ldots \ldots \ldots \ldots \ldots \ldots$

3-8 Along-track correlations after the removal of the barotropic signal from a numerical model (solid line), and without the correction (broken line). Curves from a) $24 \mathrm{SE}$ at $-58.1^{\circ}$ lon $29.7^{\circ}$ lat; b) $74 \mathrm{NW}-45.3^{\circ}$ lon, $26.8^{\circ}$ lat; c) $100 \mathrm{NW}-53.8^{\circ} \mathrm{lon}, 26.8^{\circ}$ lat; and d) $76 \mathrm{NW}$ at $-51^{\circ}$ lon, $26.8^{\circ}$ lat. . .

3-9 Along-track correlation for cross over of transects 126 and 65 (-69.4 ${ }^{\circ}$ lon, $39.1^{\circ}$ lat). Solid lines show the correlation after the orbit error correction while broken lines show the original correlation. The dotted line corresponds to the lowest value that is significantly different from zero.

3-10 Sea surface height variance from TOPEX/Poseidon data (repeat cycles to ) near the Gulf Stream. Dotted lines show the areas selected for computing 2-dimensional correlations. . . . . . . . . . . . . .

3-11 Two-dimensional correlation computed from TOPEX/Poseidon data for the $5^{\circ}$ by $5^{\circ}$ bins along the Gulf Stream. . . . . . . . . . . . 
3-12 Same as Figure 3-11 but for the bins to the south of the Gulf Stream

3-13 Confidence intervals in $\log _{10}$ for the sea surface height correlations along the Gulf Stream (Figure $3-11$ ) . . . . . . . . . . 51

3-14 Confidence intervals in $\log _{10}$ for the sea surface height correlations to the south of the Gulf Stream (Figure 3-12) . . . . . . . . . 52

3-15 Two-dimensional covariance computed from TOPEX/Poseidon data for the $5^{\circ}$ by $5^{\circ}$ bins along the Gulf Stream. . . . . . . . . 53

3-16 Same as Figure 3-16 but for the bins to the south of the Gulf Stream.

3-17 (a) Sea surface height correlation for a $5^{\circ}$ by $5^{\circ}$ area, (b) the proposed representation of the correlation as the product of a cosine times a gaussian function (see text) and (c) the residual of the least squares fit. 56

3-18 (Sea surface height correlation when point along the same transect have been included (a), and when they have been avoided (b). b) Logarithm of the confidence intervals for a), and d) logarithm of confidence intervals when same-arc points are not used. . . . . . . . . 58

4-1 TOPEX/Poseidon transects employed to sample the numerical simulation data . . . . . . . . . . . . . . . . .

4-2 Eddy kinetic energy in $m^{2} s^{-2}$ computed from (a) surface velocity of the numerical simulation, (b) geostrophic velocity derived from POX sea surface height, and (c) geostrophic velocity derived from TOPEX/Poseidon sea surface height data. . . . . . . . . . . . . . . . 64

4-3 Variance ellipses for the model data sampled as TOPEX/Poseidon (solid line) and for TOPEX/Poseidon data (dashdot line) . . . . . . 
4-4 Along track correlation for the numerical simulation sampled along TOPEX/Poseidon transects, at the cross over formed by arcs 176 and 191 (-56.6 ${ }^{\circ}$ lon, $37.11^{\circ}$ lat). Directions (a) South-East (solid lines) and North-West (dash-dot line) for arc 176, and (b) North-East (solid line) and South-West (dash-dot line) for arc 191. Dotted line at the smallest value significantly different from zero $\ldots \ldots \ldots \ldots \ldots$

4-5 Along track correlation for the numerical model data sampled as TOPEX/P where high correlation values appear at distance from the cross over. Crossover by arcs 74 and $115\left(-55.2^{\circ}\right.$ lon and $42.97^{\circ}$ lat $)$. Directions a) South-East (solid lines) and North-West (broken line) for arc 74, and b) North-East (solid line) and South-West (broken line) for arc 115 . Dotted line at the smallest value significantly different from zero. . . 68

4-6 Sea surface height variance from the POP model. Dotted lines show the areas selected for computing the 2-dimensional correlations The Gulf stream influence is identified by the high variability values. . . .

4-7 Two dimensional covariance using all the numerical model data . . .

4-8 Two dimensional covariance for the POP model when sampled along the TOPEX/Poseidon's ground tracks. . . . . . . . . . . 72

4-9 Two dimensional correlation using all the numerical model data . . .

4-10 Two dimensional correlation for the POP model when sampled along the TOPEX/Poseidon's ground tracks. . . . . . . . . . . . .

4-11 Confidence intervals in $\log _{10}$ for the correlations computed with the numerical simulation's sea surface height sampled along the satellite's groundtracks (Figure $4-10) \ldots \ldots \ldots \ldots$. . . . . . . .

4-12 3-dimensional view of the sea surface height correlation for the model f1 area (a), the proposed representation (see text) (b), c) contours of (a), contours of $(\mathrm{b})$, and the residual of the least square fit. . . . . . 
5-1 Stream function's standard deviation from a) TOPEX/Poseidon data and b) the analytic form. Contours above $25000 \mathrm{~cm}^{2} \mathrm{~s}^{-1} \ldots \ldots \ldots 94$

5-2 transport in sverdrups $\left(1 \times 10^{6} \mathrm{~m}^{3} \mathrm{~s}^{-1}\right)$ forced by the eddy vorticity flux when the stream function correlation of the bin g2 of the TOPEX/Poseidon is used. The isotropic component (a), component for anisotropy in meridional or zonal direction (b), and the diagonal anisotropy compo-

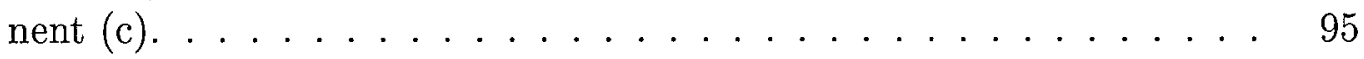

5-3 transport in sverdrups $\left(1 \times 10^{6} \mathrm{~m}^{3} \mathrm{~s}^{-1}\right)$ forced by the eddy vorticity flux when the stream function correlation of the bin $\mathrm{f} 1$ of the numerical simulation is used. The isotropic component (a), component for anisotropy in meridional or zonal direction (b), and the diagonal anisotropy component (c) . . . . . . . . . . . . . . . . . . 97

A-1 Marquardt diagram for Tapered Least Squares (solid line) and Singular Value Decomposition (dots) . . . . . . . . . . . . . . . . . . 112 


\section{Chapter 1}

\section{Introduction}

\subsection{Eddy Field- Mean Flow Interaction}

The large scale ocean circulation has, almost everywhere, a broad spectrum of intense low-frequency fluctuations generally exceeding, in strength, the mean flow. The mesoscale range, comprising time scales between 20 and 150 days, and spatial scales between 50 and $500 \mathrm{~km}$, is the dominant part of the spectrum for some regions (Stammer and Böning, 1996). The mesoscale variability, referred as eddy field in this work, is important as it interacts with the mean flow, and it contributes in the transport of heat, momentum and tracers in the ocean. It is important to understand how the eddy field interacts with the mean flow in order to comprehend the properties of the ocean transport and its impact on climate.

One of the first features of the variability to be known was the inhomogeneity of the eddy energy spatial distribution in the oceans. High values are present in the vicinity of strong flows, such as western boundary currents and the Antarctic Circumpolar Current, and low values in the subtropical gyres and in the eastern side of the basins (Stammer and Böning, 1996). Bottom topography, where it interacts with strong currents, also influences the spatial distribution of the variability (e.g. Morrow et al. 1994). 
Eddies and their associated fluxes can be essential in determining the character of the mean flow, not only as a dissipative mechanism, but also as a driving force through nonlinear interactions between eddies, and between eddies and the mean flow (Webster, 1961). We would expect these eddy driven flows to be important near the western boundary currents.

Many efforts have been devoted to the study of the eddy variability in the oceans for the last 30 years or so (see Wunsch, 1981 for a good historical review of the first works). Some studies have been conducted with observations obtained from current meter arrays (e.g. Hogg, 1988; Bower and Hogg, 1992), XBT data (e.g. Nishida and White, 1982), satellite tracked drifting buoys (Richardson, 1983) and satellite altimetry using different satellites: SeaSat (e.g. Fu and Chelton, 1985), Geosat (e.g. Tai and White, 1990; Stammer and Böning, 1992; Morrow et al., 1994) and TOPEX/Posidon (Wunsch and Stammer, 1995; Stammer, 1997) among others.

The altimetric measurements have the advantage over local in-situ observations in that they can cover whole ocean basins with nearly synoptic resolution in time and space, while the local observations provide measurements from a few points. The sparse coverage of the in-situ observations, combined with the inhomogeneous spatial distribution of the variability, can lead to erroneous generalization which can be avoided with satellite studies. The advantage of the satellite use for variability studies was pointed out 20 years ago (Wunsch, 1981), and we now have better satellites for studying oceanic variability. TOPEX/Poseidon, the first space mission specifically designed for studying ocean circulation through sea surface topography measurements, was launched on August 10, 1992. It still provides good quality data after nine years of being launched.

The ocean eddy field can be studied from the sea surface topography assuming geostrophic balance(see section 5.1). The inferred variability from TOPEX/Poseidon corresponds mainly to the barotropic and first baroclinic modes (Wunsch, 1997). It then seems plausible to use TOPEX/Poseidon data and get a good sense of the 


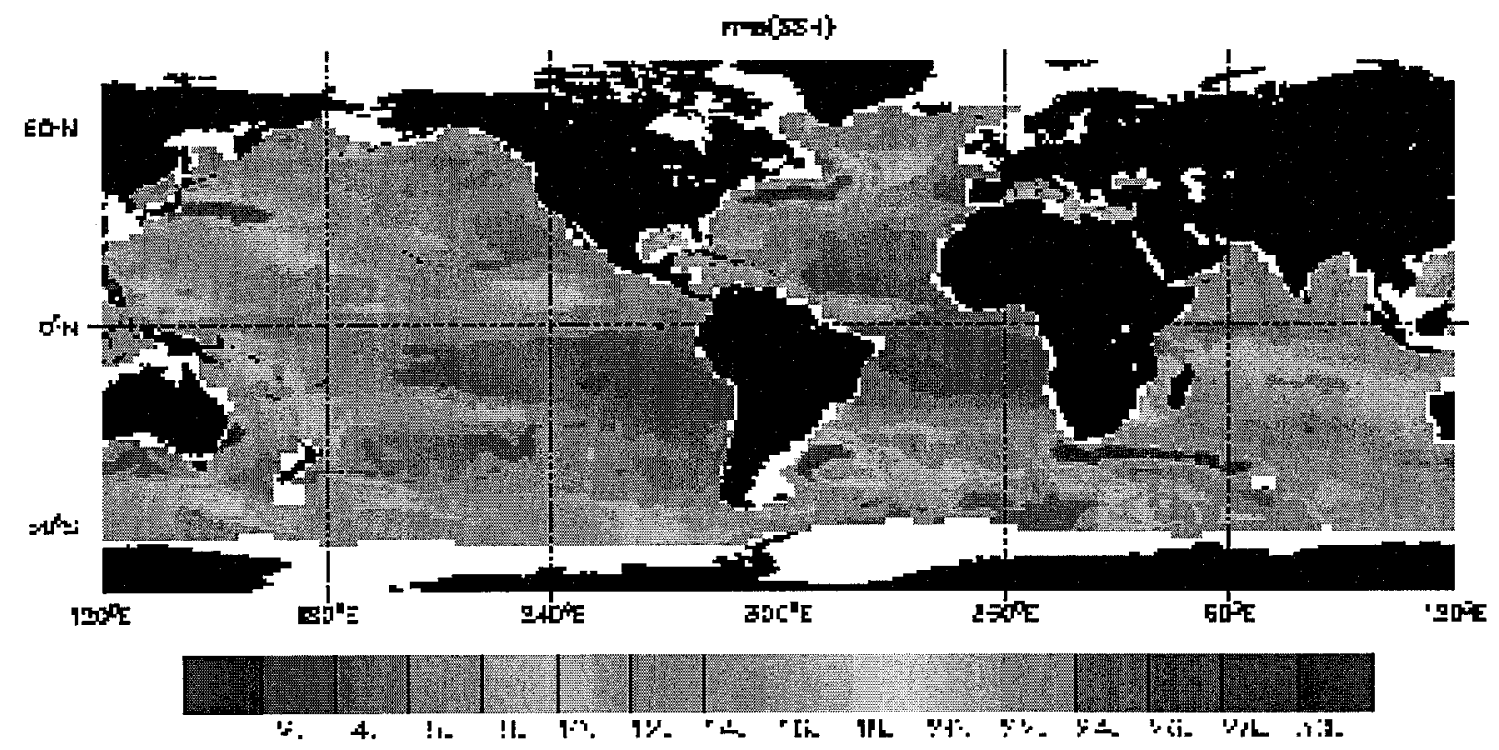

Figure 1-1: Root mean square for TOPEX/Poseidon's sea surface height in $\mathrm{cm}$ (repeat cycles 8 to 112 ).

ocean circulation for regions where the barotropic mode is dominant. Rhines (1977) suggested the barotropic mode is dominant in the high energetic regions of the oceans such as the western boundary currents. Observational studies seem to corroborate this: the region near the Gulf Stream has been observed to be mainly barotropic (Schmitz, 1980; Wunsch, 1997) as well as the Aghulas Current (Wunsch, 1997) and the Brazil and Malvinas Currents at the Confluence Zone (Peterson, 1992; Saunders and King, 1995).

Western boundary currents are easily identified by their high values on a map of sea surface height root mean square as constructed from satellite observations (figure 1-1)

The off diagonal component of the velocity covariance, the Reynolds stress per unit mass (from now Reynolds stress) have been observed to be generally positive to the south of the current and negative to the north for both, the Gulf Stream and the Kuroshio Current ( Webster, 1961; Schmitz, 1982; Hogg, 1988; Tai and White, 1990), which means that the mean current is being strengthened by the convergence of the Reynold Stress. Most of the explanations given for this distribution implicate 


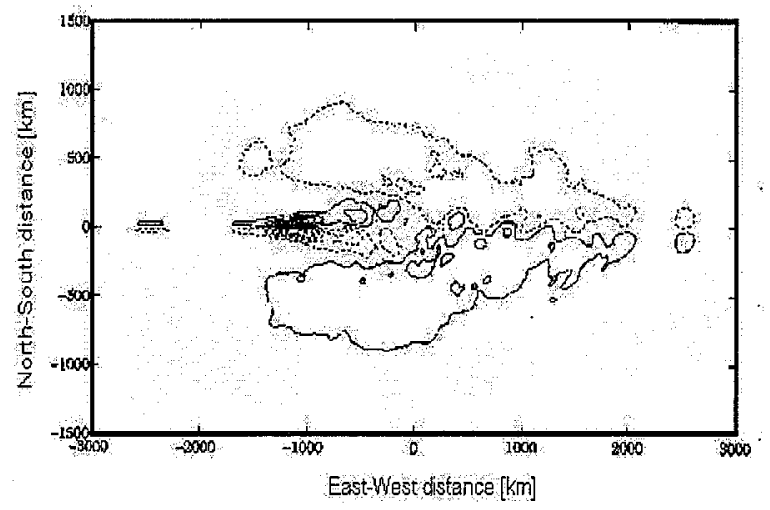

Figure 1-2: Contours of $\overline{u^{\prime} v^{\prime}}$ from a barotropic quasigeostrophic model. A zonal jet is imosed as boundary condition on the East and West boundaries. Contour interval is $0.001 \mathrm{~m}^{2} s_{2}$. Solid lines are positive and dashed lines are negative. From Jayne et al. (1996)

Rossby waves excited by the meandering of the current which polarize the eddy field (Tai and White, 1990). Jayne et al. (1996) observed a region with the same Reynolds stress distribution when studying a zonal jet with a barotropic and quasigeostrophic model, but they also observed, upstream from this region, a zone where the pattern is reversed; positive Reynolds stress to the north of the current and negative to the south (figure 1-2). This distribution seems to be produced by barotropic instability according to Jayne et al. (1993)

Features on the mean circulation near the western boundary currents have also been observed, such as recirculation gyres of opposite sign to the north and south of the Gulf Stream (e.g. Hogg, 1983) and an anticyclonic cell in the Argentine Basin above a topographic feature known as the Zapiola Drift (Saunders and King, 1995). One explanation for the recirculation cells, (e.g. Hogg, 1988; Malanotte-Rizzoli et al. 1995 ), depends on the flux of potential vorticity by the eddy field through a mean potential vorticity balance of the form,

$$
\overline{\mathbf{u}} \cdot \nabla \bar{q}=-\nabla \cdot\left(\overline{\mathbf{u}^{\prime} q^{\prime}}\right)
$$

where $\mathbf{u}=(u, v)$ is the horizontal velocity vector and $q$ the potential vorticity. 
The overbar indicates time average and the prime eddy quantities, $\mathbf{u}=\overline{\mathbf{u}}+\mathbf{u}^{\prime}$.

Hogg (1993) proposed a statistical method which allows one, under certain assumptions, to infer the various moments of the eddy field from the stream function covariance. He found, using measurements from two long-term moored arrays in and near the Gulf Stream, that the divergence of the relative vorticity flux is capable of driving two counter-rotating gyres of $30-40 S v$ on either side of the current.

Jayne et al. (1996) compared eddy moments computed from their quasigeostrophic model with those inferred by using Hogg's statistical method and found a good qualitative agreement, although the statistical method gives smaller amplitudes of $\overline{u^{\prime} v^{\prime}}$.

The use of the stream function covariance and correlations in the study of the interaction between the eddy field and the mean flow is extended in this study to include anisotropic correlations. It will be shown that small departures from the isotropy have a large impact on the eddy vorticity flux. The stream function correlation is obtained from the altimetric data and compared with the one obtained from an eddy resolving numerical model.

A description of satellite altimetry and the numerical model are provided in the second chapter. The third chapter describes the statistical methods used with the TOPEX/Poseidon data. Chapter four describes the data processing and results of the numerical simulation. In Chapter five, the method for computing the eddy fluxes in terms of the stream function statistics, obtained in chapters 3 and 4 , is described. Discussion and conclusions are presented in chapter six 


\section{Chapter 2}

\section{Satellite Altimetry and Numerical}

\section{Model}

The principle of satellite altimetry and the corrections needed to make it useful in oceanography are reviewed in this chapter with the intention of providing a general background on the subject. A description of the numerical model output used in this work is also given.

\subsection{Satellite Altimetry.}

In the absence of all forces other than gravity and the centrifugal force due to the earth's rotation, the surface of the oceans would conform to an equipotential surface, called geopotential or marine geoid. The sea surface height can then be defined as the sea surface deviation from the marine geoid. Since the ocean currents on time scales longer than a few days and space scales longer than a few tens of kilometers are very nearly in geostrophic balance, save close to the equator $(<100 \mathrm{~km})$, the current velocity can be calculated from the pressure gradient on the geoid (see section 5.1 for the mathematical development). In accord with the hydrostatic approximation, the pressure gradient at the geoid is proportional to the horizontal slope of the sea 
surface. Satellite altimeter observations of sea surface topography can then provide global information of the ocean surface velocity (Chelton et al. 2001)

The French-US altimetric satellite TOPEX/Poseidon, launched in August 1992, has been very successful in providing global scale measurements with a repeat period of 9.9 days, nominally a "10-day cycle", in which it orbits the earth 127 times. It orbits the earth at an altitude of $1336 \mathrm{~km}$ and an inclination of $66^{\circ}$. This orbiting configuration results in a ground track separation of about $316 \mathrm{~km}$ at the equator (Fu et al., 1994).

The principle for measuring the sea surface height with satellite altimeter is simple: obtain the distance between the satellite and the earth's surface, called the range, and subtract it from the distance between the satellite and the geoid (figure2-1). The sea surface height obtained can be decomposed into the dynamic sea-surface height associated with geostrophic currents, the height produced by tides and the sea-surface response to atmospheric pressure.

In reality, it is not that simple to measure the sea surface height using the altimeter, as can be seen in the brief description that follows; a more detailed one can be found in Chelton et al. (2001).

\subsubsection{Errors and Corrections.}

The altimeter measures the time it takes a microwave pulse to travel from the satellite to the earth's surface and back. The range can not be obtained directly from the time measurement because there are processes along the ray's path that affect the travel time and shape of the microwave pulse.

Some constituents of the atmosphere (i.e., water vapor, dry gases and free electrons in the atmosphere) reduce the propagation speed of the microwave pulse. It is necessary to correct for these delays before computing the range. These corrections can be made with great accuracy for the TOPEX/Poseidon data and are not considered a significant source of error (Chelton et al., 2001). 


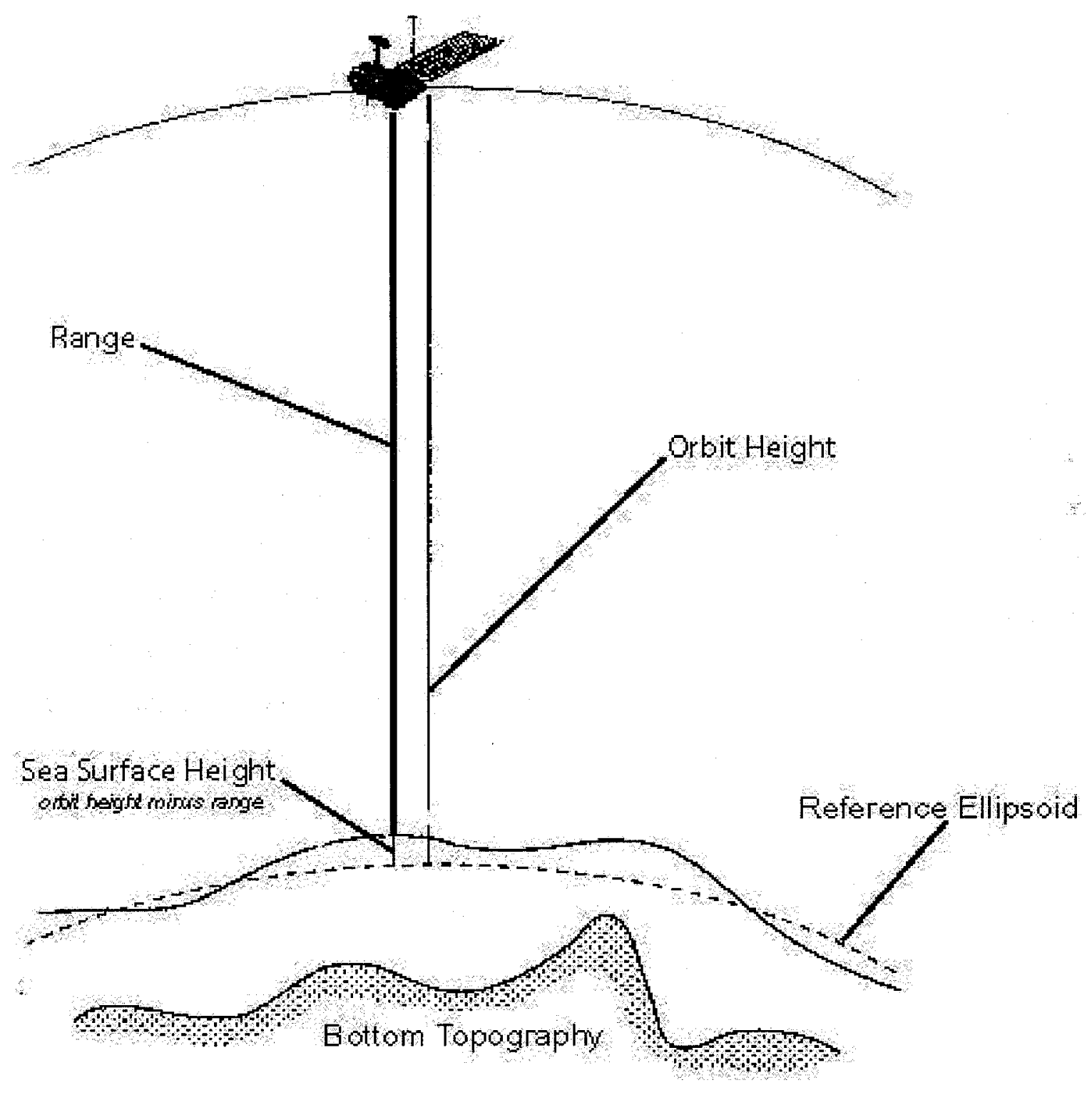

Figure 2-1: Schematic summary of the corrections that must be applied to the altimeter range measurement $R$. escanear figura 1 de Fu $\&$ Cazenave 2001 pp.2 
Another correction is needed due to the biases in the estimation of the sea state (wave and swell height, direction and period). The return signal measured by the satellite corresponds to the pulse reflected by small wave facets within the antenna footprint, called scatterers, that are perpendicular to the incident radiation. Errors arise in the determination of the sea level because of differences between the distribution of the scatterers and the sea surface height. It is also believed that this correction can be done very accurately for TOPEX/Poseidon (Chelton et al. 2001).

Once the range is known, the distance between the satellite and the geoid needs to be found. What is known of the satellite's orbit is the orbit height or altitude $(\mathrm{H})$, the distance between the satellite and the geopotential surface. The geopotential surface is, in fact, approximated by an ellipse; the geoid has undulations in the range of $-105 \mathrm{~m}$ to $+85 \mathrm{~m}$ from the reference ellipse due to gravitational anomalies owing to variations in the bottom topography and in-homogeneities in the earth's interior. Therefore, an independent estimate of the orbit height and the geoid departure from the reference ellipsoid are needed.

Two new sources of errors appear: imprecisions in the modelling of the true shape of the geoid, referred to as the geoid problem, and inaccuracy in the calculation of the three-dimensional location of the satellite with respect to a specified reference frame, called the orbital ephemerides.

The geoid problem can be bypassed in the study of mesoscale variability by subtracting the time mean of the data since the time scales of geoid changes are much longer than the time scales of oceanic mesoscale variability. The reduction in the uncertainty in the orbit ephemerides, mainly its radial component, known as the orbit error, is considered a particular success of the TOPEX/Poseidon mission as it has been reduced to about $2 \mathrm{~cm}$. It is believed that no orbit error correction is needed at this level (Chelton et al. 2001). An in-depth description of the orbit error and its correction is given in Appendix A since it was thought to be important at one point in the progress of this work. 
The sea surface height obtained is a mix of different components, such as tides and atmospheric load, that need to be taken into account in order to procure the geostrophic portion.

The TOPEX/Poseidon mission made significant improvements in the development of a tidal model. The tidal potential can be approximated by six components, all of which are diurnal or semi-diurnal in most regions of the world. The repeat period of the satellite is longer than the tidal periods so the latter aliases into lower frequencies that are of interest for ocean circulation studies, and therefore need to be removed. For TOPEX/Poseidon, the orbit configuration was selected so the energetic tidal constituents would alias to periods that can be well resolved using a few years of altimeter data and that can be easily distinguished from other narrow banded ocean signals, such as the annual and semiannual. The result is a reduction of the tidal model inaccuracy from more than $10 \mathrm{~cm}$,prior to TOPEX/Poseidon, to $2-3 \mathrm{~cm}$.

The atmospheric pressure exerts a downward force on the sea surface to which the sea surface responds to first order in a static manner. The atmospheric loading has spatial and temporal variations and so does the sea surface response. The conventional wisdom establishes that an increase (decrease) in atmospheric pressure of 1 mbar depresses (raises) the sea level by about $1 \mathrm{~cm}$; this is called the "inverted barometer" correction. The small dynamical response of the sea level to the atmospheric loading generates errors that are probably on the order of $10 \%$ of the correction. The accuracy of the inverted barometer correction is also limited by the uncertainty in the knowledge of the sea surface atmospheric pressure, which can be as large as $2-3 m b a r$ in the tropics and sub-tropics, $4 \mathrm{mbar}$ north of $40^{\circ} \mathrm{N}$ and $4-7 \mathrm{mbar}$ south of $40^{\circ} \mathrm{S}$.

The TOPEX/Poseidon measurements are believed to be at the $2-3 \mathrm{~cm}$ level of accuracy after the described corrections are made (see TOPEX/Poseidon special issues of the Journal of Geophysical Research. December 1994 and December 1995). 


\subsection{Numerical Model}

The numerical simulation of the North Atlantic Ocean at $0.1^{\circ}$ of resolution, using the Los Alamos Parallel Ocean Program (Smith et. al., 2000) is considered in this study. At $0.1^{\circ}$ of resolution $(3.2 \mathrm{~km}$ in the horizontal at the northern boundary and $11.1 \mathrm{~km}$ at the equator) this is an eddy resolving simulation. A description of the model and the simulation are given below paraphrasing Smith et al. (2000).

The Los Alamos Parallel Ocean Program (POP) is a level-coordinate ocean general circulation model that solves the three dimensional primitive equations with realistic bottom topography. It is a Bryan-Cox type model (Bryan, 1969) with implicit freesurface treatment of the barotropic equations (Dukowicz and Smith, 1994).

The domain of the $0.1^{\circ}$ extends from $20^{\circ} \mathrm{S}$ to $72.6^{\circ}$, and $98^{\circ} \mathrm{W}$ to $17.2^{\circ} \mathrm{E}$. The horizontal resolution varies from $11.1 \mathrm{~km}$ at the equator to $3.2 \mathrm{~km}$ at higher latitudes. Smith et al. (2000) show that this horizontal grid spacing is less than or equal to the zonal-mean Rossby deformation radius at all latitudes. The eddies should then be reasonably well resolved in the model at all latitudes since the typical length scales for mesoscale eddies are related to Rossby radius as showed by Stammer (1997).

The simulation was produced by forcing the model with realistic winds (ECMWF TOGA Global Surface Analysis) in the period of 1985-1996. The model was initialized with the ocean at rest; the temperature and salinity were set equal to the June Levitus (1982) climatology. The model was spun up for 5.3 years, after which the wind stress was switched back to October 1, 1985 and continued through July 1, 1996.

The data were archived every 10 model days, roughly the same sampling period of TOPEX/Poseidon, as snapshots of the three dimensional prognostic variables.

The $0.1^{\circ}$ resolution improved many features in the North Atlantic simulation. over previous runs at coarser resolution One of the most relevant is that the mesoscale eddy field appears stronger and closer to observations than in previous simulations, particularly in quieter regions, such as the interior of the subtropical gyre and in the eastern basin ( Smith et al. 2000). The Gulf stream, which had proven particularly 
difficult to simulate realistically in previous runs, has also major improvements. It separates from the boundary at Cape Hatteras, rather than a few hundred kilometers to the north, as it had done in other numerical studies, and it separates as a jet without the strong anticyclonic eddy at the boundary that characterizes many previous simulations. The peak velocities, transports, spatial scales, and structures in the Gulf stream extension agree well with current meter data. (Smith et al. 2000)

The simulated Gulf stream also shows differences with respect to observations. It flows in a more zonal direction after the separation than observed, resulting in a southward offset of the mean path of $1^{\circ}-1.5^{\circ}$ in latitude. It also presents a broader meridional extent, particularly near the New England Sea Mounts (65 and $57^{\circ} \mathrm{W}$ ).

Smith et al. (2000) compare the sea surface height from the model with satellite data from a blended TOPEX/Poseidon and the European Space Agency's ERS-1 and $E R S$-2. The satellite variability was computed from $0.25^{\circ} \times 0.25^{\circ}$ maps covering a two-year period from April 1995 to April 1997 every ten days, as that was the longest continuous multiyear period available.

When comparing the sea surface height from the model with TOPEX/Poseidon's, Smith et al. (2000) found that the altimetric data shows regions (i.e., North American coast southwest of Nova Scotia and off the South American coast between $0^{\circ}$ and $5^{\circ} \mathrm{N}$ ) of very high variability that do not appear in the model which they explain as residual errors in the tidal model. They also found that in the regions of low sea surface height variability, such as in the western basins and the tropics, the satellite variability is higher than in the model. They point out that this might be due to the noise in the observations 


\section{Chapter 3}

\section{TOPEX/Poseidon}

The altimetric data provides measurements of the sea surface height along the satellite's groundtracks. The data needs to be processed with statistical methods in order to obtain as much information as possible and understand it. In this chapter, a description of the data, the statistical methods and its results are described. Part of the information obtained in this chapter, particularly the standard deviation and the correlation of the sea surface height, is used in chapter 5 to compute the eddy vorticity flux. Other statistical quantities are compared in chapter 4 with the statistical quantities of the numerical method.

\subsection{Data Processing.}

The MIT TOPEX/POSEIDON data set is used in this study for the region given by $-75^{\circ}$ and $-45^{\circ}$ lon and $25^{\circ}$ and $45^{\circ}$ lat. Figure (3-1) shows the satellite groundtracks that are used. The data have been corrected for all environmental effects and interpolated onto a fixed $6 \mathrm{~km}$ equidistant along track grid (King et al., 1994). Data points at depths shallower than $1000 \mathrm{~m}$ are discarded as the tidal correction is not reliable at shallow depths. The data set presents gaps in which the satellite did not work, and some points which appear to be incorrect. To deal with this, points different from the 


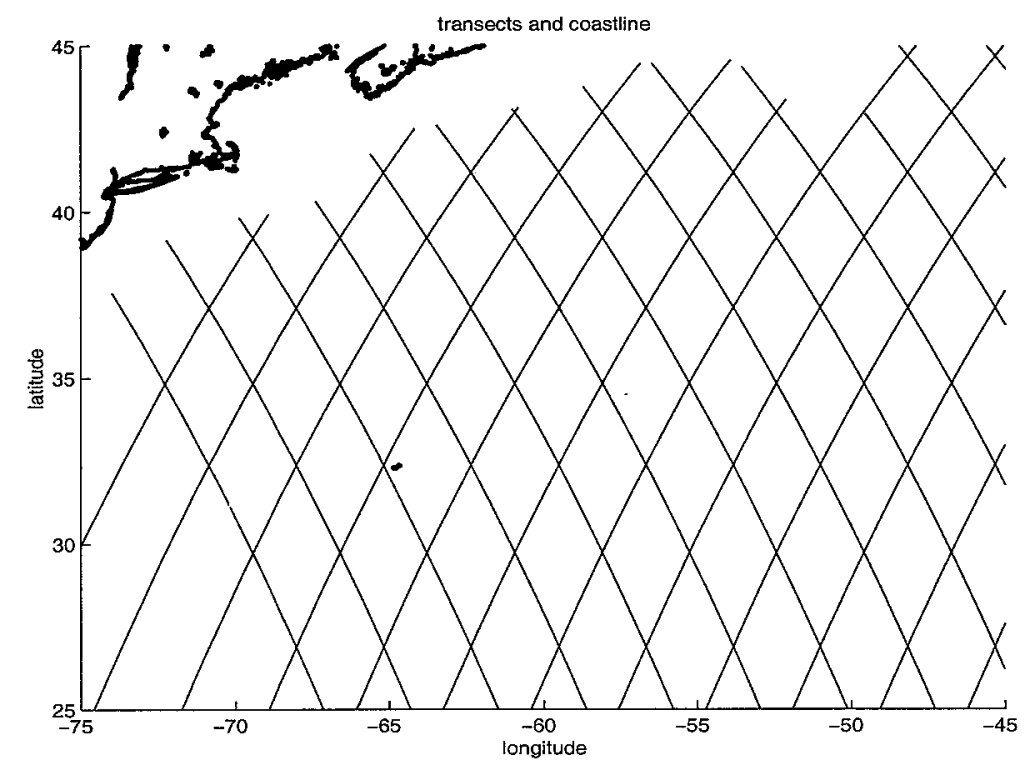

Figure 3-1: TOPEX/Poseidon ground tracks in the Gulf Stream area

mean by three standard deviations are identified as outliers and eliminated, although it should be acknowledge that there is some risk that these points correspond to real signals in the sea surface. The gaps with no data are then filled with a spline cubic interpolator (MATLAB). Each arc is first interpolated in space and then in time, with the limit in the gap size of $48 \mathrm{~km}$ in space (eight points) and 11 days in time (one point). The remaining gaps become smaller so a new round of interpolations can be performed. This is done for three interpolation cycles, eliminating the outliers after each round.

The interpolated data are filtered with a butterworth filter (MATLAB) in time and space. The Nyquist frequency, defined as one-half of the sampling frequency and that it corresponds to the highest frequency that can accurately be resolved, is used as the low-pass cutoff frequency in the time filter to remove the noise (higher frequencies that can not be resolved). For the space filtering, a $30 \mathrm{~km}$ wavelength is used as suggested by Stammer (1997). 


\subsection{Eddy Kinetic Energy and Ellipse Variances}

The geostrophic perturbation velocity can be computed at the cross overs of the satellite arcs assuming geostrophic balance (see Section 5.1). It is important to remember that the velocities obtained are the perturbation velocity since the time mean of the sea surface height has been eliminated. Let $\bar{u}$ be the time mean of $u$,

$$
\mathbf{u}^{\prime}(\mathbf{x})=\mathbf{u}(\mathbf{x}, t)-\overline{\mathbf{u}}(\mathbf{x})
$$

The groundtracks are divided into ascending (from Northwest to Southeast, labelled with odd numbers) and descending (from Southwest to Northeast, labelled with even numbers). To compute the geostrophic velocity, the alongtrack sea surface height gradient is obtained ( $\gamma_{a}$ and $\gamma_{d}$ for ascending and descending arcs) and transform into the zonal and meridional sea surface height gradients $\left(\gamma_{m}, \gamma_{z}\right.$ respectively) following Parke et al. (1987),

$$
\begin{aligned}
\gamma_{m} & =\frac{\gamma_{a}+\gamma_{d}}{2 \cos (\theta)} \\
\gamma_{z} & =\frac{\gamma_{a}-\gamma_{d}}{2 \sin (\theta)}
\end{aligned}
$$

The geostrophic perturbation velocity is obtained from the sea surface height gradient (see Section 5.1 for the derivation of this expression)

$$
\epsilon_{i k j} f u_{j}^{\prime} \hat{k}=-\frac{1}{\rho} \frac{\partial \eta}{\partial x_{i}},
$$

where $\rho$ is the density of the water, $\epsilon_{i k j}$ is the alternating tensor, $f$ is the Coriolis parameter due to the earth's rotation, and $\hat{k}$ is the unitarian vector in the vertical direction. Once the meridional and zonal components of the velocity are obtained at each cross over, the eddy kinetic energy can be found, $0.5\left(\overline{u^{\prime 2}}+\overline{v^{\prime 2}}\right)$, where the overbar stands for time average (figure 3-2). Stammer (1993) obtain the global eddy kinetic energy map using the TOPEX/Poseidon data, but with a different method. He computes the geostrophic velocity between each pair of points along the arcs and assumed the velocity to be isotropic. The velocity is not assumed isotropic in 

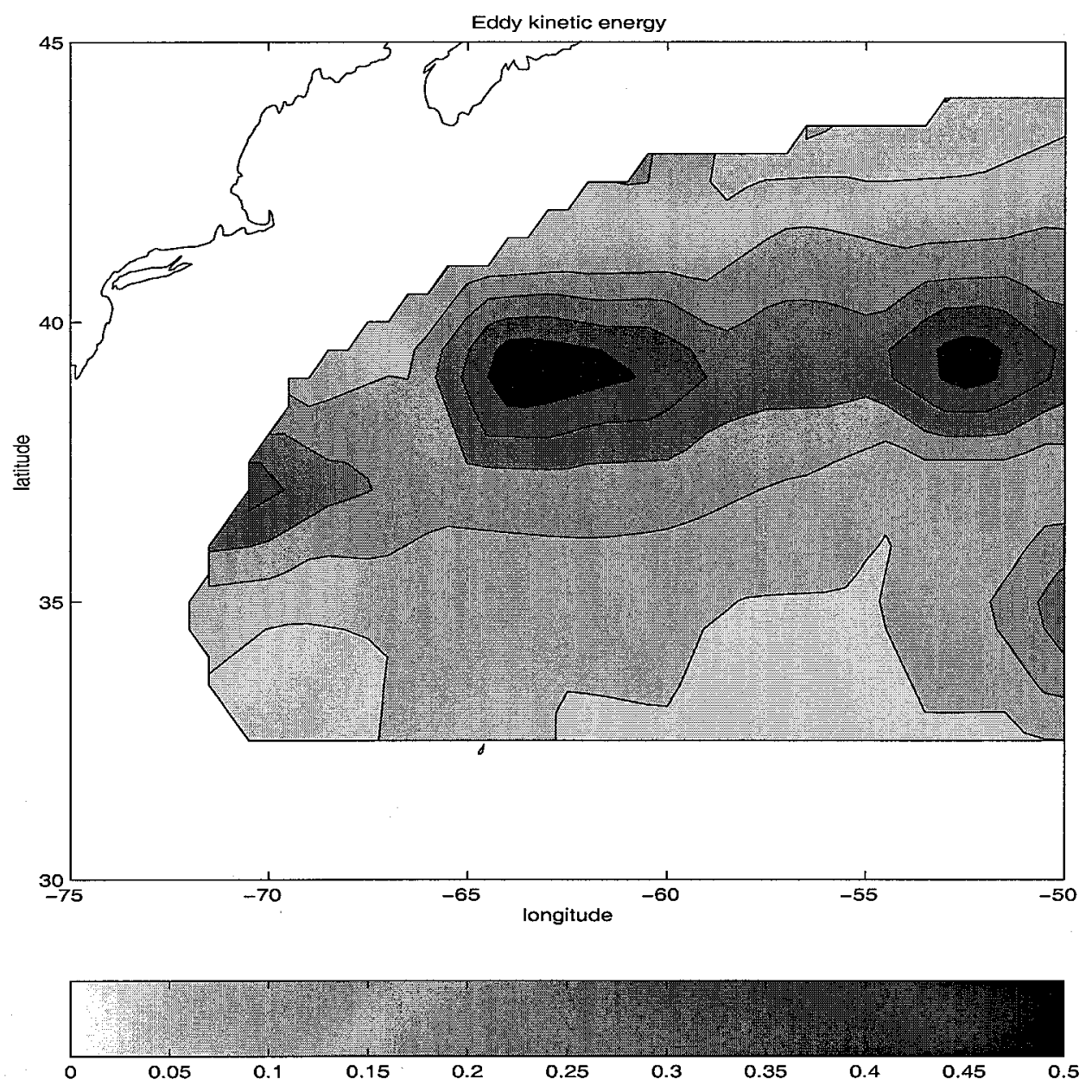

Figure 3-2: Eddy kinetic energy computed from the geostrophic velocity at the satellite's cross overs

this study; both horizontal velocity components are found for each crossover. The downside of this method is that it diminishes the resolution. In section (4.1) this issue is addressed by comparing the information obtained from the velocity field of the numerical model and the one provided by the geostrophic velocities computed at the cross over from the simulation's sea surface height field. The ellipses of variance are computed from the velocity covariance, $\overline{u_{i}^{\prime} u_{j}^{\prime}}$, as a way to represent the magnitude and the direction of the variability. The direction is given by the angle $\theta$ (Preisendorfer, 1988),

$$
\theta=\frac{1}{2} \arctan \left(2 \frac{\overline{u_{1}^{\prime} u_{2}^{\prime}}}{\overline{u_{1}^{\prime 2}}-\overline{u_{2}^{\prime 2}}}\right)
$$


the variance along the semimajor axis by

$$
\sigma_{11}=\frac{1}{2}\left(\overline{u_{1}^{\prime 2}}+\overline{u_{2}^{\prime 2}}+\sqrt{\left(\overline{u_{1}^{\prime 2}}-\overline{u_{2}^{\prime 2}}\right)^{2}+4\left(\overline{u_{1}^{\prime} u_{2}^{\prime}}\right)^{2}}\right)
$$

and the variance along the semiminor axis is

$$
\sigma_{22}=\frac{1}{2}\left(\overline{u_{1}^{\prime 2}}+\overline{u_{2}^{\prime 2}}-\sqrt{\left(\overline{u_{1}^{\prime 2}}-\overline{u_{2}^{\prime 2}}\right)^{2}+4\left(\overline{u_{1}^{\prime} u_{2}^{\prime}}\right)^{2}}\right) .
$$

The points on the ellipse are function of the angle $\phi=0: 2 * \pi$. In cartesian coordinates, the ellipses take the form

$$
\begin{gathered}
x=\sigma_{11} \cos (\phi) \cos (\theta)-\sigma_{22} \sin (\phi) \sin (\theta) \\
y=\sigma_{11} \cos (\phi) \sin (\theta)+\sigma_{22} \sin (\phi) \cos (\theta)
\end{gathered}
$$

Isotropic flows have circles instead of ellipses, since $\overline{u_{1}^{\prime 2}}=\overline{u_{2}^{\prime 2}}$, and $\overline{u_{1}^{\prime} u_{2}^{\prime}}=0$. Anisotropic flow is represented by elongated ellipses, whose orientation is given by the velocity covariance, $\overline{u_{1}^{\prime} u_{2}^{\prime}}$. The semimajor axis indicates the principal direction of the variance.

Figure 3-3 shows the variance ellipses computed form the altimeter data. The ellipses have been normalized by the largest semimajor axis (0.6586). The Gulf Stream can be identified by the larger ellipses. There ellipses indicate that the velocity near the Gulf Stream is not completely isotropic, as they are not circles. There is not a preferable direction to which the ellipses align. Maybe a more structured pattern could be seen if the crossovers had a better resolution.

\subsection{Alongtrack Correlations}

The TOPEX/Poseidon data provide a time series of sea surface height every six kilometers. A correlation between those time series with zero time lag can be used to see how similar they are, and at what distance the sea surface height value of one point is not influenced by the value at the other position, that is, at what distance 


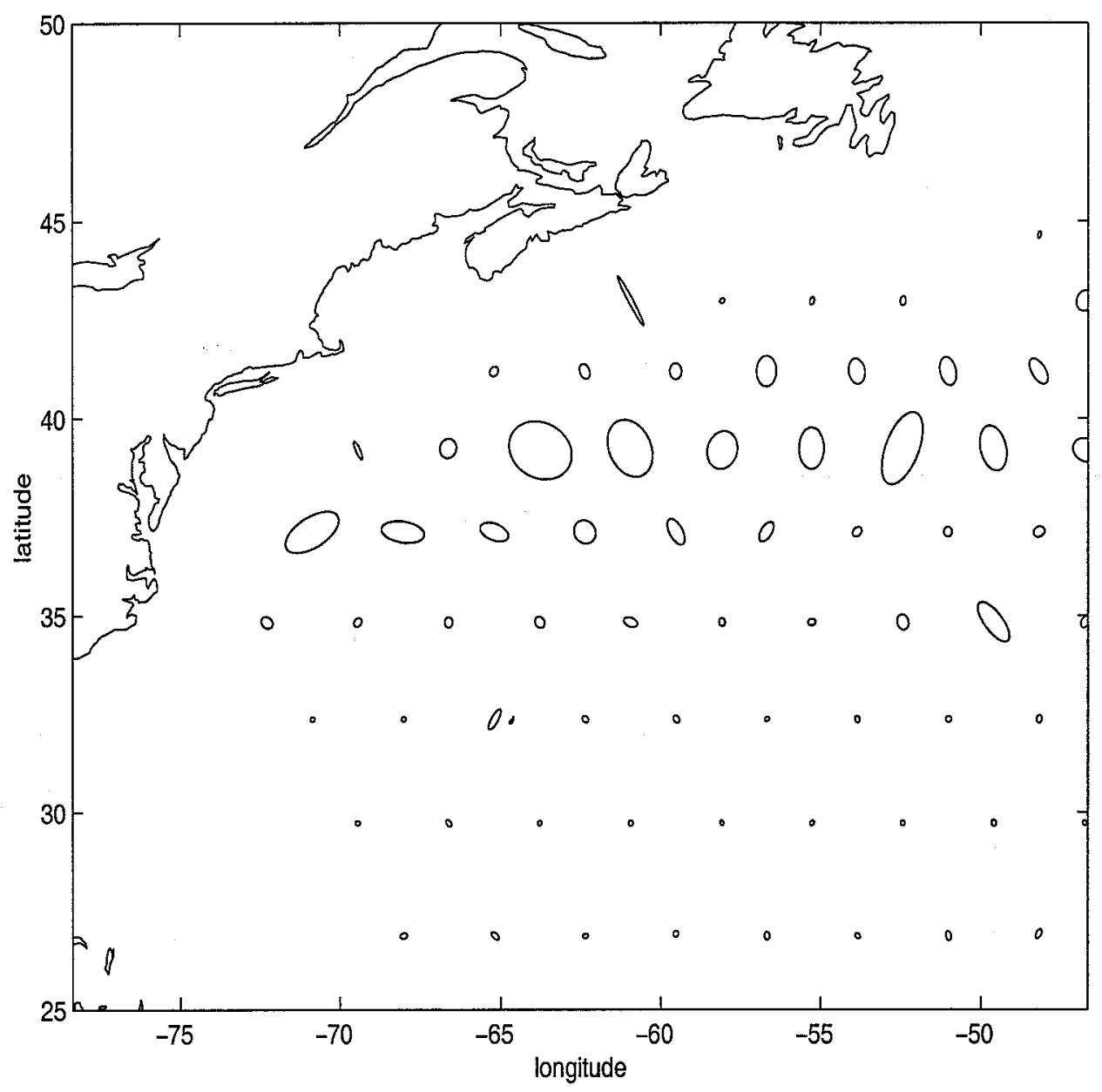

Figure 3-3: Variance ellipses from TOPEX/Poseidon data, normalized with maximum semimayor axis 
they are independent. If one time series is kept fixed, and the correlation is computed with time series of the rest of the points along the same arc, the correlation can be seen as a function of distance along the arc or space lag. It is necessary to compute the covariance first in order to compute the correlation. The covariance is,

$$
\operatorname{Cov}(\delta s)=\frac{1}{N-1} \sum_{j=1}^{N}\left(h\left(s, t_{j}\right)-\mu\left(t_{j}\right)\right)\left(h\left(s+\delta s, t_{j}\right)-\mu\left(t_{j}\right)\right),
$$

where $h\left(s, t_{j}\right)$ is the sea surface height time series at the cross over, $N$ the number of time realizations, and $h\left(s+\delta s, t_{j}\right)$ is the time series at a distance $\delta s$ along the same transect. The spatial mean along the arc at each particular time is given by $\mu\left(t_{j}\right)$. The convention for $\delta s$ is that it can only be positive. The correlation is obtained by normalizing the covariance by the square root of the product of the variances,

$$
C(\delta s)=\frac{\operatorname{Cov}(\delta s)}{(V(s) V(s+\delta s))^{\frac{1}{2}}}
$$

where $V(s)$ and $V(s+\delta s)$ are the variances,

$$
V(s)=\frac{1}{N_{t}-1} \sum_{j=1}^{N_{t}}\left(h\left(s, t_{j}\right)-\mu\left(t_{j}\right)^{2}\right) .
$$

Note that the method described for computing the covariance differs from the one normally used for a multidimensional process in which the value of the field depends on more than one variable. The covariance for a two dimensional process is written as (Priestly, 1981),

$\operatorname{Cov}(\delta s, \delta t)=\frac{1}{N-1} \frac{1}{M} \sum_{i=1}^{M-\delta s} \sum_{j=1}^{N-\delta t}\left(h\left(s_{i}, t_{j}\right)-E[h(s, t)]\right)\left(h\left(s_{i}+\delta s, t_{j}+\delta t\right)-E[h(s, t)]\right)$,

where $h\left(s_{i}, t_{j}\right)$ is the sea surface height measured at the position $s_{i}$ and time $t_{j} . M$ is the number of points in space and $N$ the number of time realizations. $E[h(s, t)]$ is the expected value of the sea surface height. These calculations use almost all the points in a transect to compute the covariance value at one space lag, while the one in this study takes only two timeseries for one space lag. The correlation computed using 


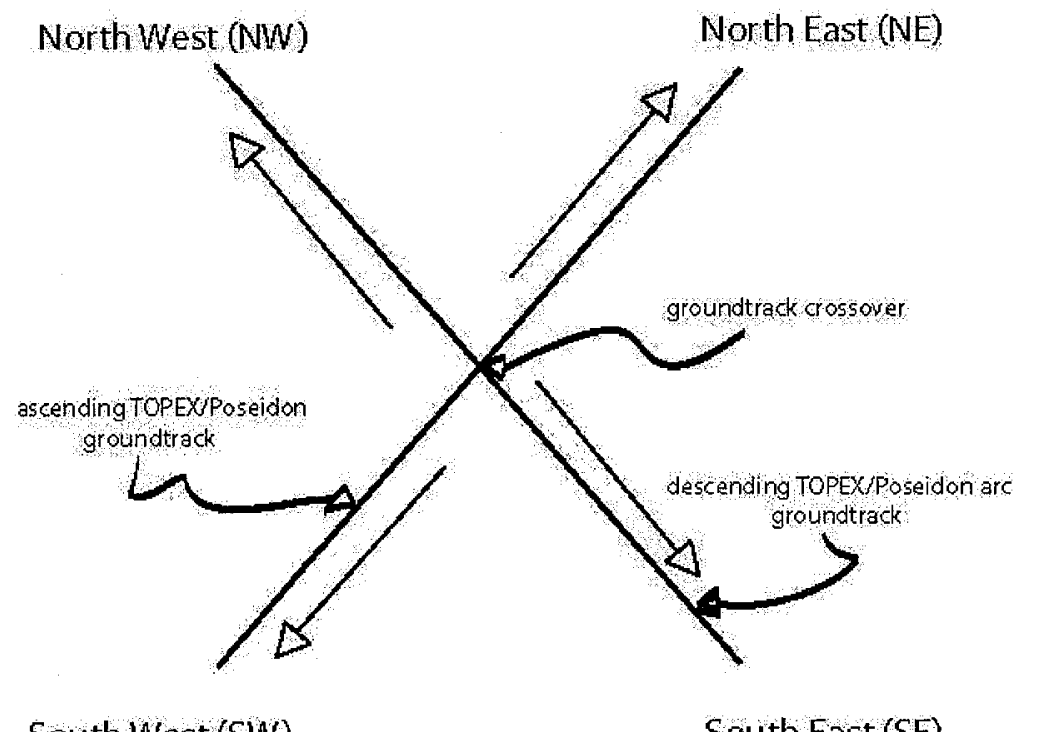

South West (SW)

South East (SE)

Figure 3-4: scheme of the directions for the alongtrack correlations.

equation 3.11 is more robust than the one used here since it involves more data points in the calculation reducing the error bars. I nevertheless decided to use equation 3.8 so that the influence of one particular point in the arcs with respect to the rest can be isolated. A correlation more similar to the robust one is computed in section 3.4.

For an isotropic process, the correlation is independent of the direction in which the correlation is taken. The isotropy of the correlation for the sea surface height field will be verified by comparing the correlations obtained in different directions. In order to have more directions available, the transects can be broken in two at the location were two arcs cross. For each cross over, four series are then available, two per arc, pointing in four directions that are labelled with respect to the cardinal point toward which the correlation is done (Figure 3-4). The correlation is computed by each time series at the crossover with points that belong to the same arc. This correlation does not involve two time series of different arcs.

One of the features that can aid in the comparison of the correlation in the four directions is the correlation length scale, the distance at which the correlation crosses zero. The correlation length scale is sought in each direction by searching for the 
first point that is not significantly different from zero (Sokal \& Rohlf, 1981). It is expected that the correlation would decline gently towards zero with distance from the crossover, and to oscillate around zero after certain distance, like an uncorrelated process with white noise. For an isotropic process, the correlation does not depend on the direction it is taken, so the four correlations associated with a cross over would look very similar. The correlations at certain crossovers look quite isotropic, even when considering the error bars in the computation. Figure 3-5 shows the correlations for two cross overs, one formed by arcs 74 and 89 (panels a and b), and the other one by and 176 and 191 (panels $\mathrm{c}$ and d). The dotted line indicates the smallest positive value of the correlation that can be significantly different from zero (Sokal and Rohlf ,1981 ). This gives an idea of the error bars. The correlation scales are fairly similar, and in all cases it ends oscillating around zero. This suggests an isotropic correlation. Other correlations do not decrease to zero as expected, but instead decrease monotonically at a slow rate. Some curves decline so slowly that they do not cross zero (Figure 3-6), or the value at which they do is non distinguishable from zero, even at large distances from the crossover $(300 \mathrm{~km})$. A possible explanation for having high correlations at large distance from the crossover is that the data has some errors along each transect that introduce the structure responsible for such high correlation. Orbit error or aliasing with high frequency barotropic signals could provide such structure, so they are tested below.

\subsubsection{Barotropic Signals}

Fukumori et al. (1998), using a numerical model, presented evidence that barotropic motions with frequency higher than the sampling frequency of TOPEX/Poseidon exist in the oceans. Three regions in particular showed strong barotropic high frequency activity, two in the southern ocean and one in the northern part of the North Atlantic. Stammer and Wunsch (2000) found that the altimetric data can be corrupted by the aliasing of these high frequency motions, and suggested a method for correcting it. 
a) $\operatorname{Arc} 74$
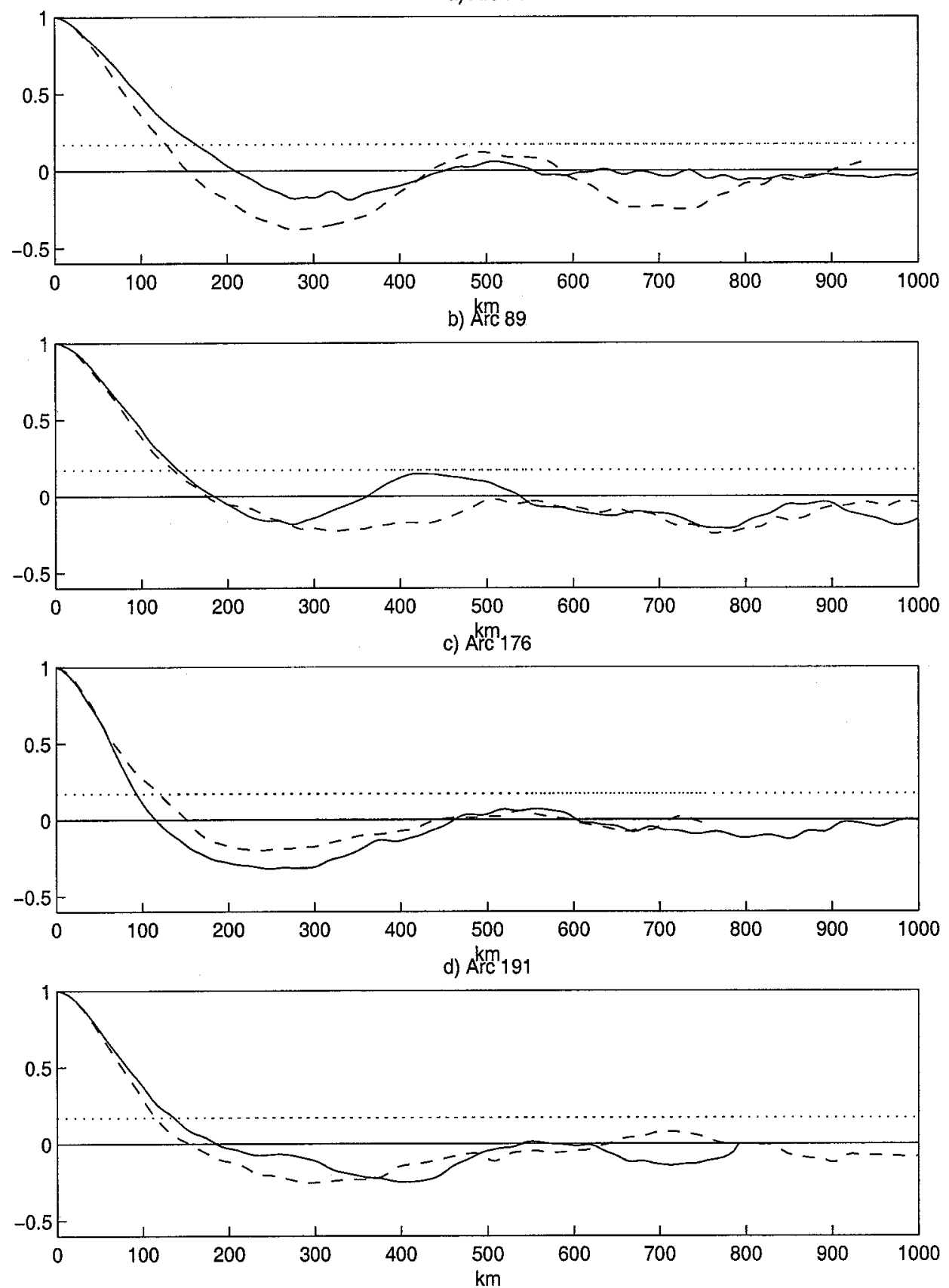

Figure 3-5: Along track correlation at cross overs formed by arcs 74 and $89\left(-51^{\circ}\right.$ lon, $37.1^{\circ}$ lat), (a) and (b), and by transects 176 and 191 ( $-56.7^{\circ}$ lon, $37.1^{\circ}$ lat), (c) and (d). The directions are SE (solid line) and NW (broken line) for even numbered arcs, (a) and (c), and NE (solid line) and SW (broken lines) for odd numbered arcs, (b) and (d). The dotted line is the smallest positive indistinguishable form zero 
a) Arc24 SE
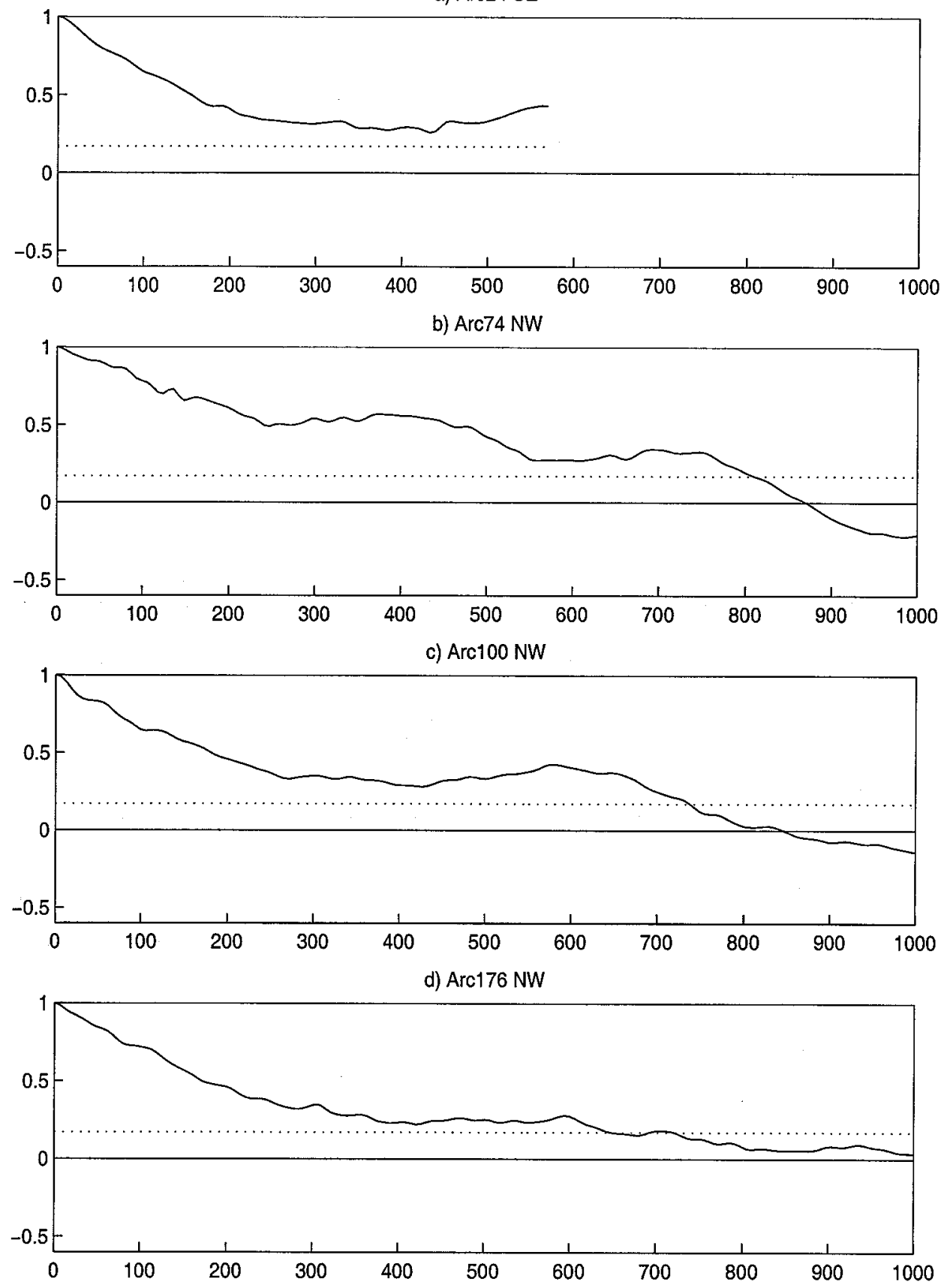

Figure 3-6: Correlation along (a) $24 \mathrm{SE}$, (b) $74 \mathrm{NW}$, (c) $100 \mathrm{NW}$, and (d) $76 \mathrm{NW}$. They correspond to the crossovers $24 / 13\left(-58.1^{\circ}\right.$ lon $29.7^{\circ}$ lat), $74 / 139\left(-45.3^{\circ}\right.$ lon, $26.8^{\circ}$ lat $)$, $100 / 165\left(-53.8^{\circ}\right.$ lon, $26.8^{\circ}$ lat), and $176 / 241$ ( $-51^{\circ}$ lon, $26.8^{\circ}$ lat) at panel d) 
In their study, the barotropic version of the MIT ocean general circulation numerical model forced twice daily by winds, reproduced with high accuracy the short period motions that the full baroclinic version of the same model with identical forcing produced. They proposed that, since the results from the two models are so similar at shorter periods than a month, and the barotropic version being easier to run, the altimetric data can be corrected by subtracting the barotropic model results from the along-track elevation.

The barotropic signal from the numerical model is removed before the filtering and the interpolation. The alongtrack correlations are computed in the same way they were obtained in the previous section. The correlations with the correction are compared to those obtained from the data with no correction.

The correction does not affect much some of the correlations. Figure 3-7 shows that the correlations for the cross over 74 and $165\left(-49.6^{\circ} \mathrm{lon}, 34.8^{\circ} \mathrm{lat}\right)$ are not significantly different. The solid line corresponds to the corrected data and the broken one to the original data.

In other instances the correction does affect the correlation. Some cases in which the correlation was found to decrease monotonically and never crossed zero, or did at large distances (Section 3.3, Figure 3-6), the correlation decays more slowly after the barotropic correction has been applied. Figure 3-8 has the same transects as Figure $3-6$; in all four cases, the correction made the slope slightly more negative. This means that the barotropic signal removal does not improve the alongtrack correlations.

The model results for the barotropic signal are available until TOPEX/Poseidon's repeat period 195. If the corrected data are to be used, the satellite's timeseries are reduced by about 80 cycles. This translates into larger error bars in the correlation. The first non-distinguishable from zero point appears at correlation 0.2 , while it was 0.17 for the uncorrected data. 
a) $\operatorname{Arc} 74 \mathrm{SE}$
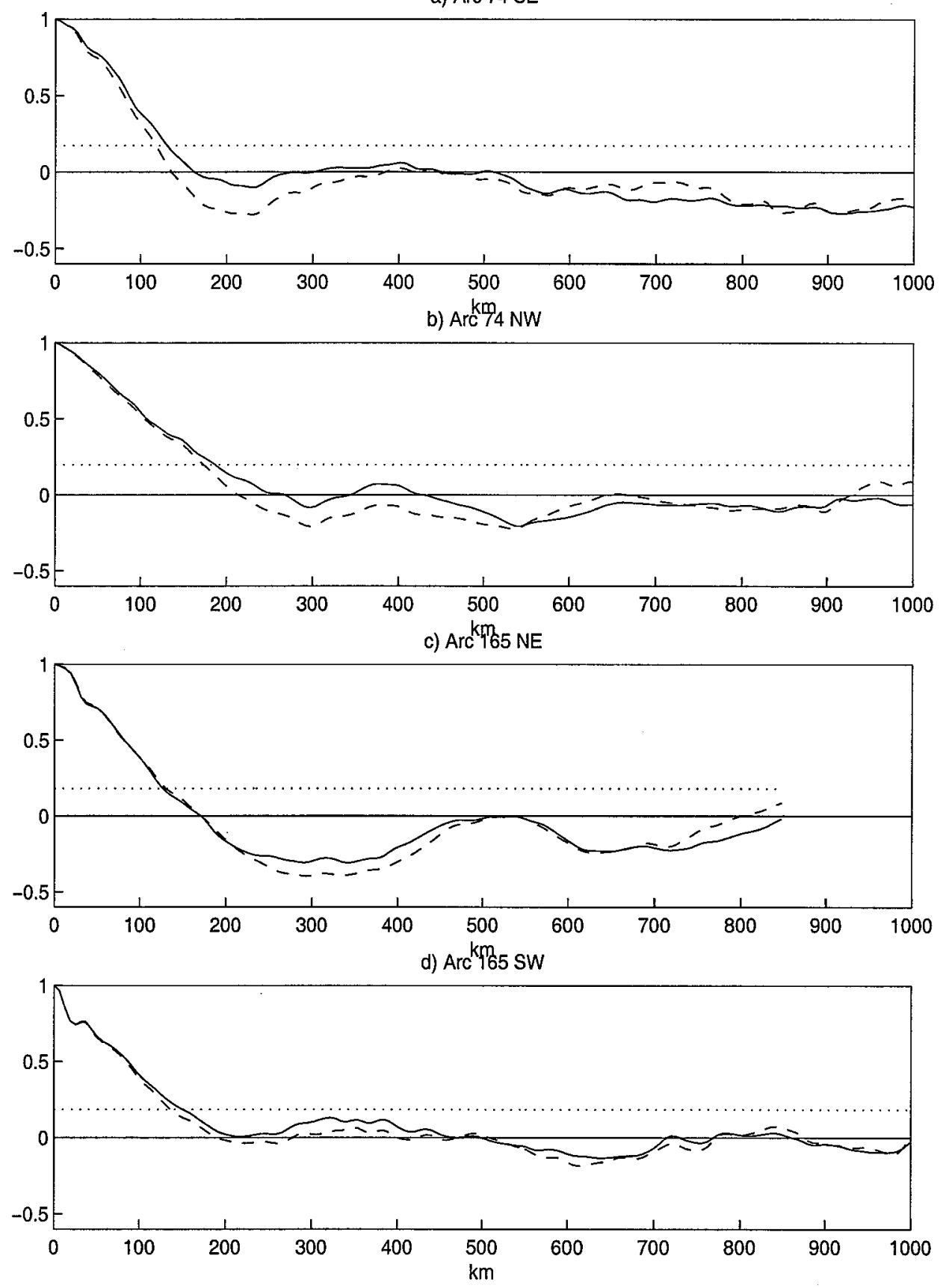

Figure 3-7: Along-track correlation at cross over made by arcs 74 and 165, after the removal of the barotropic signal from a numerical model (solid line), as proposed by Stammer and Wunsch (2000), and without the correction (broken line). The dotted line corresponds to the lowest value that is significantly different from zero 
a) $4 \mathrm{SC} 4 \mathrm{SE}$
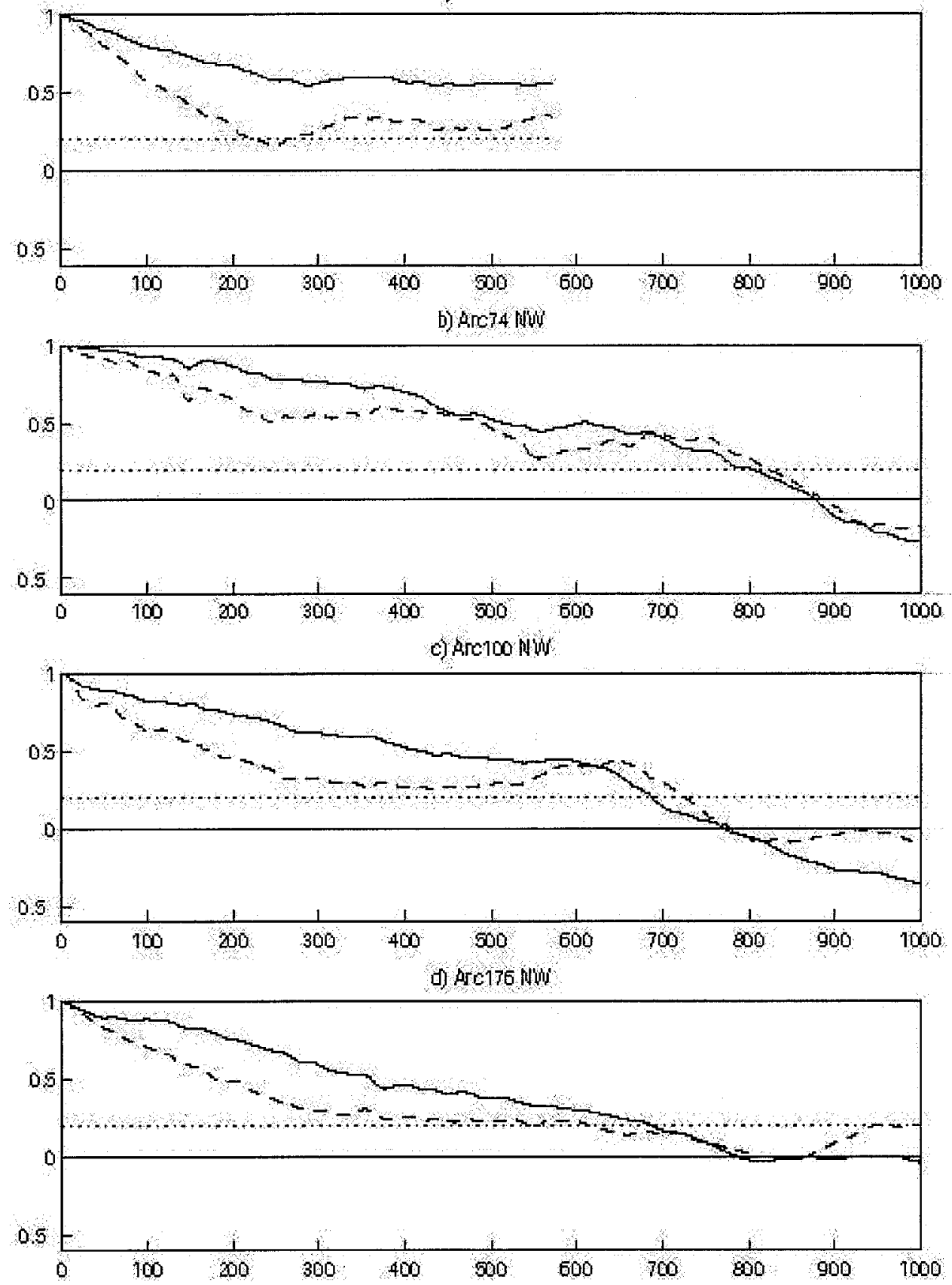

Figure 3-8: Along-track correlations after the removal of the barotropic signal from a numerical model (solid line), and without the correction (broken line). Curves from a) $24 \mathrm{SE}$ at $-58.1^{\circ}$ lon $29.7^{\circ}$ lat; b) $74 \mathrm{NW}-45.3^{\circ}$ lon, $26.8^{\circ}$ lat; c) $100 \mathrm{NW}-53.8^{\circ} \mathrm{lon}$, $26.8^{\circ} \mathrm{lat}$; and d) $76 \mathrm{NW}$ at $-51^{\circ} \mathrm{lon}, 26.8^{\circ}$ lat. 


\subsubsection{Orbit Error Correction.}

At one point it was believed that the TOPEX/Poseidon data still contained errors due to what is known as orbit error. Appendix A describes in depth the orbit error as well as the methods to correct it. In this section it is showed that the alongtrack correlations have very small changes when the correction is applied to the data.

Figure 3-9 shows the correlation for the two directions available for the crossover of the arcs arcs 126 and 65, since the transects are too small in the other two directions, with and without the orbit error correction. This crossover is where the correction produces the largest effect, and it can be seen that it is still minimal; smaller than the one obtained with the barotropic correction (figure 3-7). This supports the idea that the error induced by the high frequency barotropic motions is larger than that of the orbit error as Stammer and Wunsch (2000) mention.

It was mentioned in section 3.3 that this method for computing the correlations is not as robust as the usual one for multidimensional processes. Some unexpected features in the correlation, as the monotonically decrease of the correlation that doe not cross zero, or it crosses at a large distance form the origin, were not removed by either the orbit error correction or the removal of high frequency barotropic signals. The error bars, provided by the number of data points used in the correlation, are large. Correlations are now done in another fashion that will improve the error bars.

\subsection{Two-dimension Correlation}

The along-track covariances and correlations of the sea surface height obtained by altimetry are one-dimensional by nature. Two-dimension correlations with the resolution needed for studying the mesoscale can not be computed in the ordinary sense with the TOPEX/Poseidon data set because the spatial coverage is not fine and regular enough. In order to get two dimensional information, it is customary to map the data onto a grid using objective mapping (Bretherton et al. 1976), which has its 

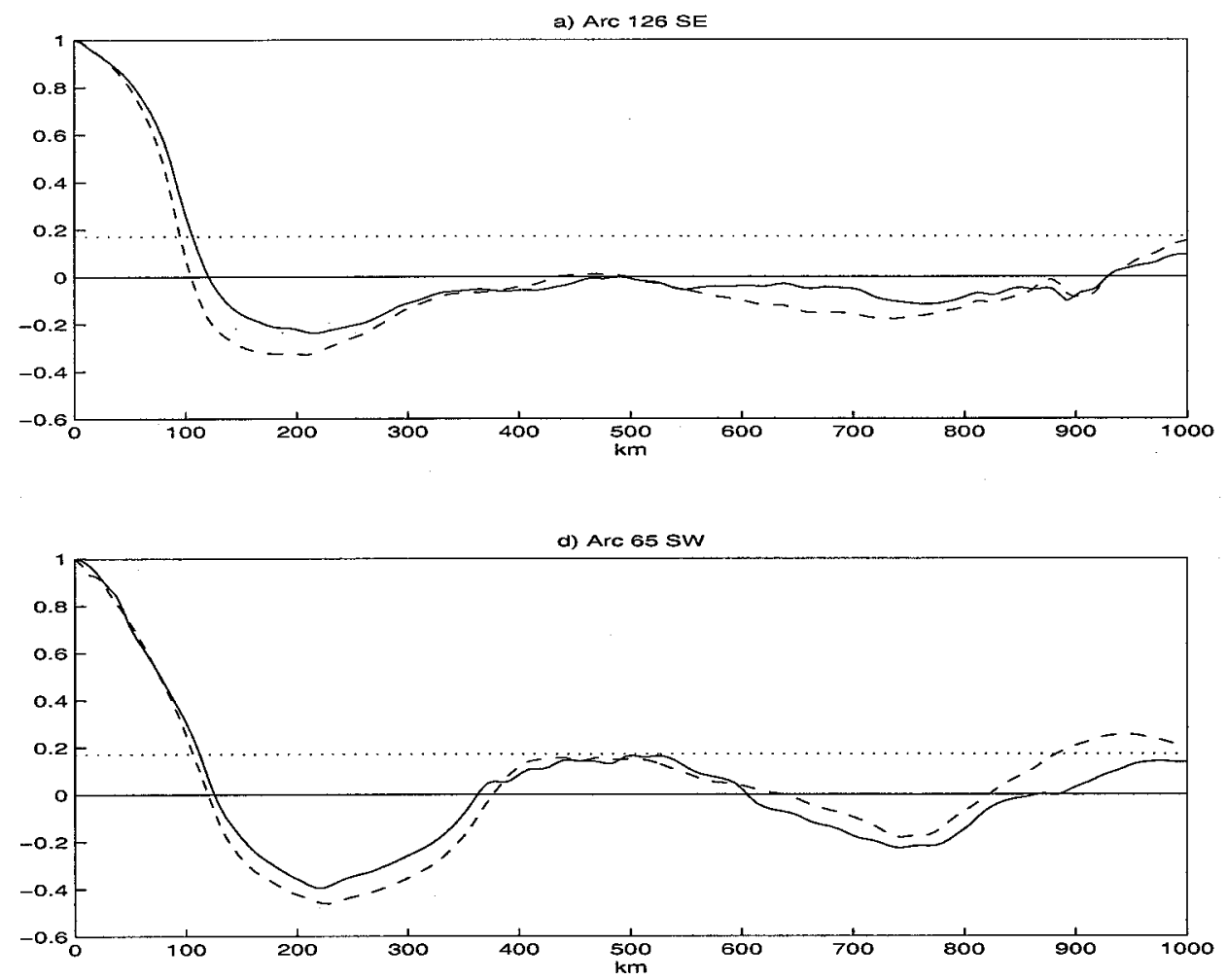

Figure 3-9: Along-track correlation for cross over of transects 126 and 65 (-69.4 $4^{\circ}$ lon, $39.1^{\circ}$ lat). Solid lines show the correlation after the orbit error correction while broken lines show the original correlation. The dotted line corresponds to the lowest value that is significantly different from zero. 
own problems (i.e., filtering of the variance, Morrow et al., (1994)). Glazman et al. (1996) introduced a method to overcome the one dimensionality of the along-track correlations without having to grid the data, although they make the assumption that the sea surface height field is statistically stationary and spatially homogeneous. The technique uses several satellite passes occurring in an area of interest, say $5^{\circ}$ by $5^{\circ}$ bin, and defines a period of time, called the synchronicity interval $\Delta t$, for which the satellite passes are treated as simultaneous. The risk of using neighboring groundtracks is that, assuming the sea surface height did not change in the time difference between the measuring of both groundtracks, it introduces errors of the magnitude of the orbit error (Parke et al. 1987). It was found that the orbit error is negligible for TOPEX/Poseidon so this is not a concern. An error can be introduced by the high frequency motions to which the sea surface height responds in the sampling time difference. This needs to be considered, but for the time being it will be assumed that the groundtracks within a synchronicity period are quasi-simultaneous. To compute the space correlation, we start by obtaining sea surface height products of pair of points from all quasi-simultaneously tracks inside the bin,

$$
\left(h\left(\mathbf{x}_{j}, t_{i}\right)\right)\left(h\left(\mathbf{x}_{j}+\mathbf{r}, t_{i}+\delta t\right)\right)
$$

where $\mathbf{r}$ is the distance vector between the two points, the time interval $\delta t$, is $\delta t \leq \Delta t$. The sea surface height field is treated as statistically stationary and spatially homogeneous; the absolute times and positions are ignored, which allows the averaging of the correlations obtained within a synchronicity interval and a particular bin to get the correlation as a function of $\mathbf{r}$,

$$
\left.C(\mathbf{r})=\frac{1}{M} \sum_{j=1}^{M} \frac{1}{N} \sum_{i=1}^{N} \frac{\left(h\left(\mathbf{x}_{j}, t_{i}\right)\right)\left(h\left(\mathbf{x}_{j}+\mathbf{r}, t_{i}\right)\right)}{V\left(\mathbf{x}_{j}\right) V\left(\mathbf{x}_{j}+\mathbf{r}\right)}\right) .
$$

where,

$$
V\left(\mathbf{x}_{j}\right)^{2}=\frac{1}{N-1} \sum_{i}^{N}\left(h\left(\mathbf{x}_{j}, t_{i}\right)\right)^{2} .
$$

The number of groundtracks available for the correlation in a particular bin is in direct proportion to the size of the bin and the length of the synchronicity interval. 
This affects the coverage in the $\mathbf{r}$ plane as well as the number of points averaged for a particular $\mathbf{r}$. The most complete uniform coverage of the $\mathbf{r}$ plane is achieved by taking the synchronicity interval to be the period of the orbit repeat cycle, 10 days for TOPEX/Poseidon mission. A downside of selecting such an interval is that, the longer the synchronicity interval is, the harder it is to satisfy the assumption of the transects being simultaneous. For this work, the 10 day period is chosen to get as many transects as possible acknowledging that there is still questions about the validity of the synchronous assumption.

The points inside the bin are paired for computing the correlation. The separation vector between them, $\mathbf{r}$, can have two directions depending on which of them is used as a reference. Both directions are taken into account in the method; each pair of points contributes twice to the calculation, and a symmetry is introduced in the averaged correlation. The points near the edges can not be paired with points in all directions within a circumference as some points lie outside the bin. To avoid this, it is allowed for the points on the edges to be coupled with points outside of the bin that are closer than $500 \mathrm{~km}$. This forms an area around the bin, called the 'fringe' area, whose points are only paired with inside points. The direction of the separation vector is unique for this case, from the inside to the outside of the bin, which somewhat breaks the symmetry that had been introduced before.

Glazman et al. (1996) decided to exclude pairs of points that belong to the same altimeter pass to eliminate the influence of fast sea surface height oscillations caused by short-correlated processes, such as gravity waves, sea state bias oscillations (due to wind speed variations) etc. The data are filtered in this study to eliminate high frequency features, so there is no need to avoid pairs of data from the same transect. Nevertheless, a comparison is made between correlations that include points from the same transect and the ones that avoid them.

The homogeneity assumption is not satisfied by the covariance in the region around the Gulf Stream. The Gulf Stream has higher variability than its surroundings, as can 
be seen in Figure 1-1. Five degrees by five degrees bins for computing the covariance and correlation are selected so that they contain points of similar regime and the homogeneity assumption is approximately valid within them. Figure (3-10) shows the sea surface height variance in the Gulf Stream region, as well as the bin positions; four bins were selected to lay along the Gulf Stream extension (labelled g1, g2 ,g3 $, g 4)$ and four to the south of it (f1, f2, f3, f4).

The correlation appears to be homogeneous and close to isotropic. Figures 311 and 3-12 show the correlation computed for the bins along the Gulf Stream and outside of it. The plots suggest that the correlations are homogeneous since the values are very similar between the different areas, and close to isotropic because they are roughly circular. The confidence intervals associated with the correlations, calculated according to Sokal and Rohlf (1981), are shown in Figures 3-13 and 3-14. The upper interval is nominally the same as the lower, so only one plot for each bin is presented. The confidence intervals are presented in $\log _{10}$ to appreciate the structure that exist because the number of points in the computation of the correlation varies significantly from point to point. The confidence intervals are quite small, with the largest values of 0.05 at the edges of the circles. The TOPEX/Poseidon transects can clearly be distinguished as they allow for more degrees of freedom by having more points in the computation.

The correlations south of the current are less circular but, save the westernmost, still appear close to isotropic. A possible explanation for the less circular shape is that the signal to noise ratio is smaller away from the Gulf Stream, given that the covariance is one order of magnitude larger along the current than out of it, (Figures $3-11$ and 3-12). The noise in the form of background variability might have a stronger influence in the southern bins and modify the otherwise close-to-circular shape.

The covariance and correlation plots do not follow a gaussian distribution; they have negative values, as can be seen in Figures 3-11, 3-12. A test of significance was performed according to Sokal and Rohlf (1961), with the caveat that the method 


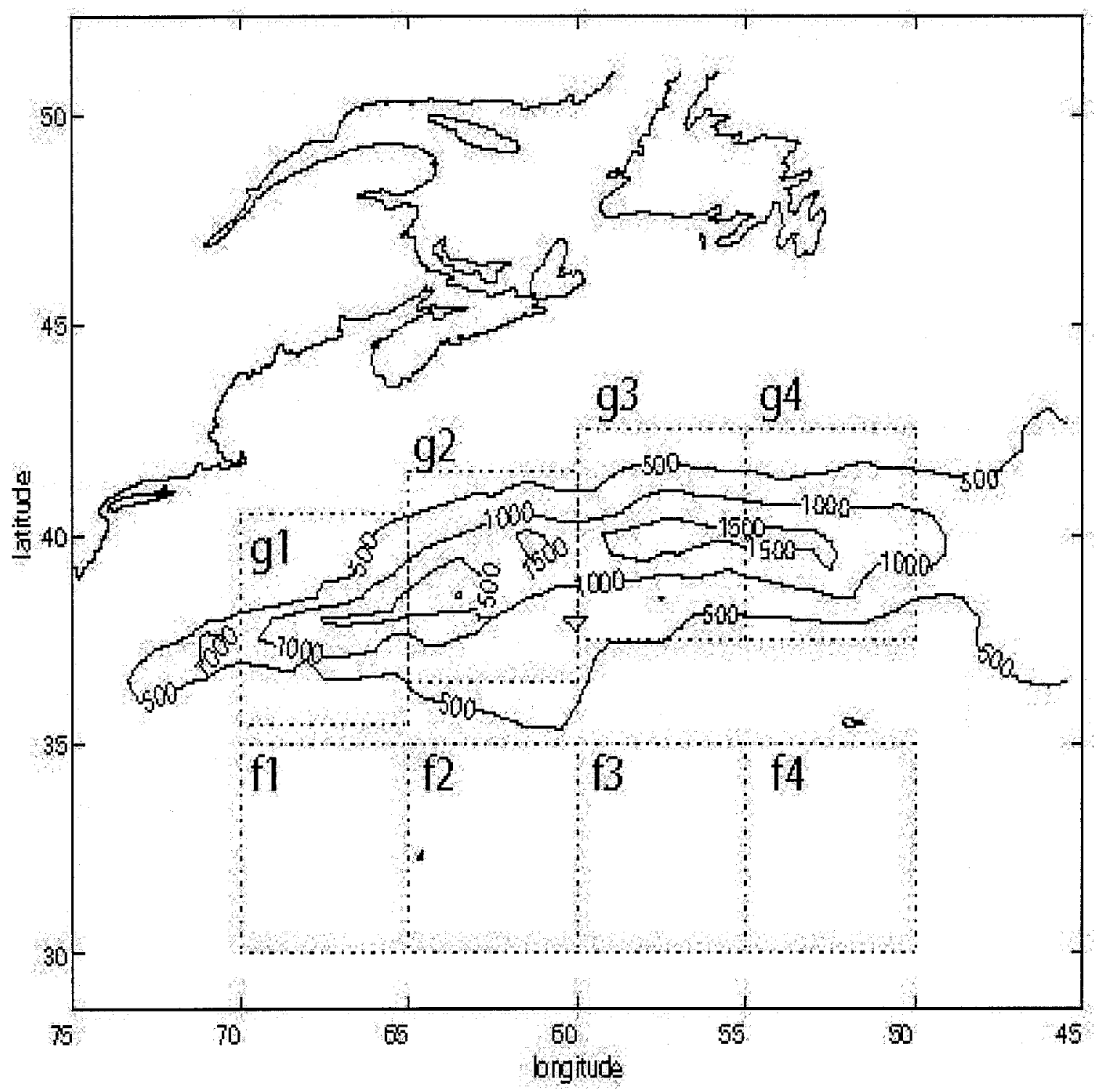

Figure 3-10: Sea surface height variance from TOPEX/Poseidon data (repeat cycles to ) near the Gulf Stream. Dotted lines show the areas selected for computing 2dimensional correlations. 

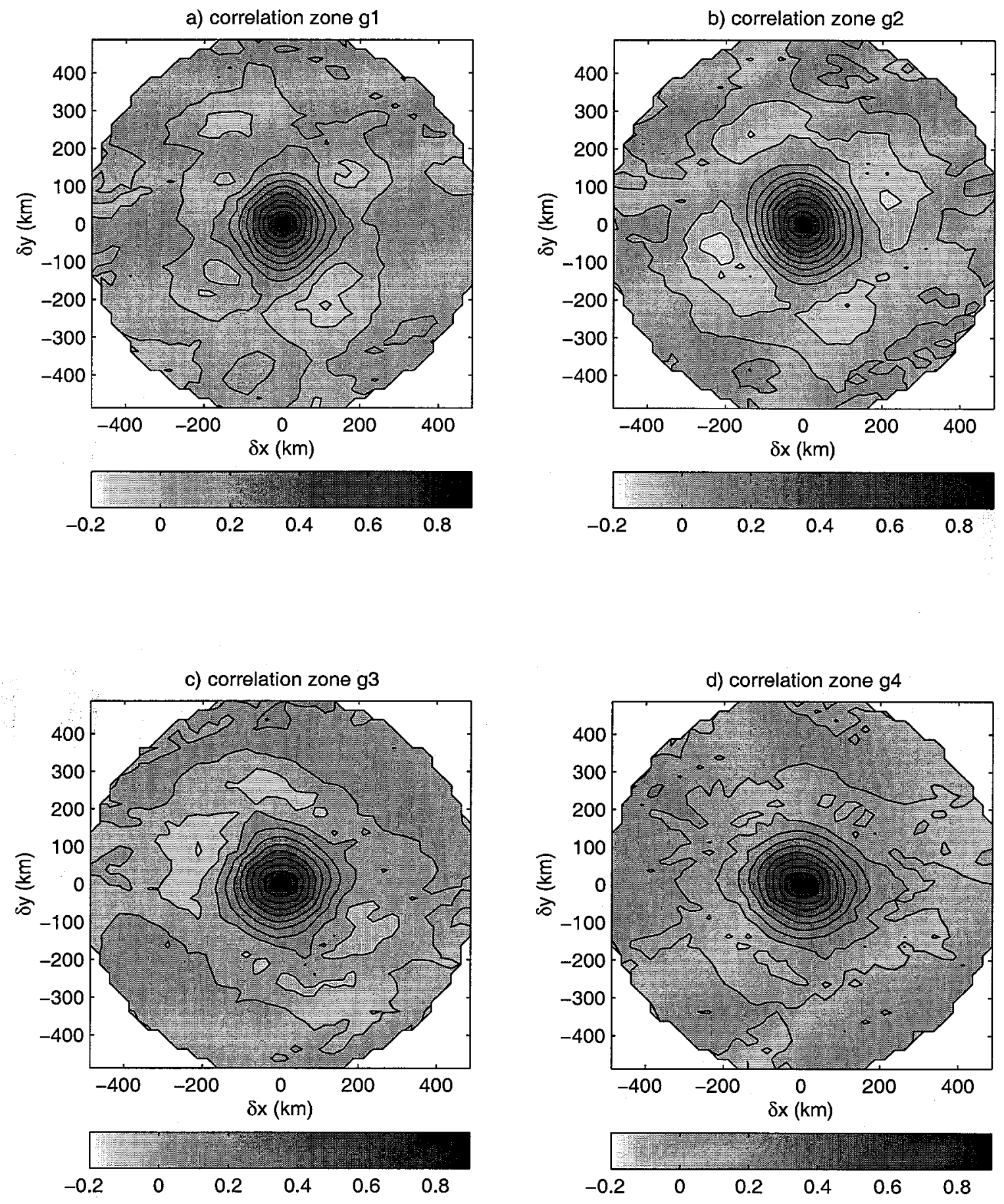

Figure 3-11: Two-dimensional correlation computed from TOPEX/Poseidon data for the $5^{\circ}$ by $5^{\circ}$ bins along the Gulf Stream. 
a) correlation zone $\mathrm{f} 1$

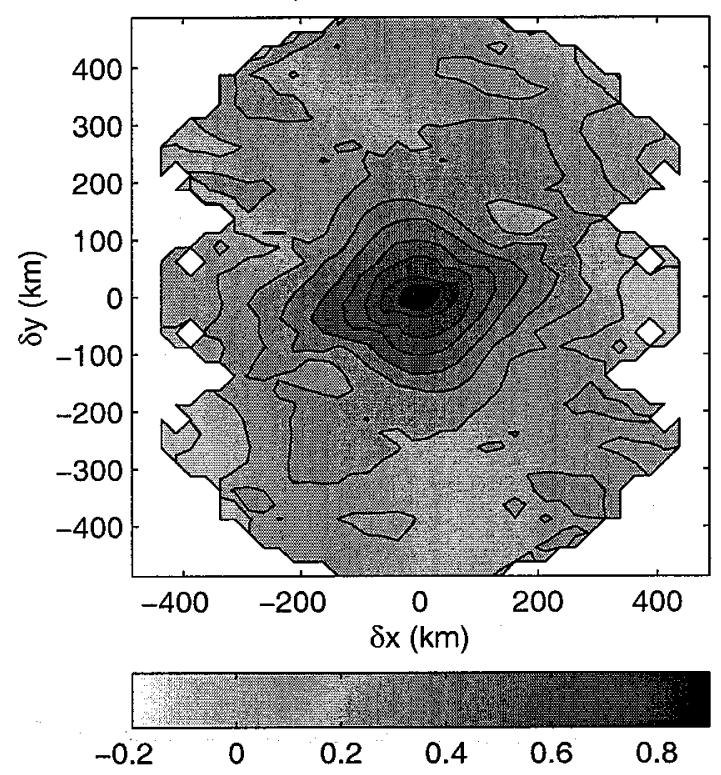

c) correlation zone $\mathrm{f} 3$

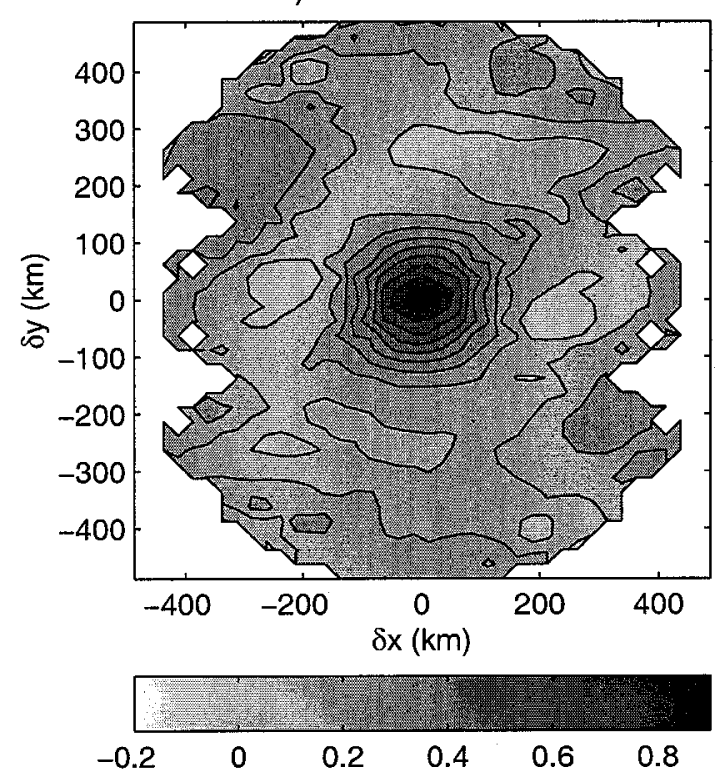

b) correlation zone $\mathrm{f} 2$

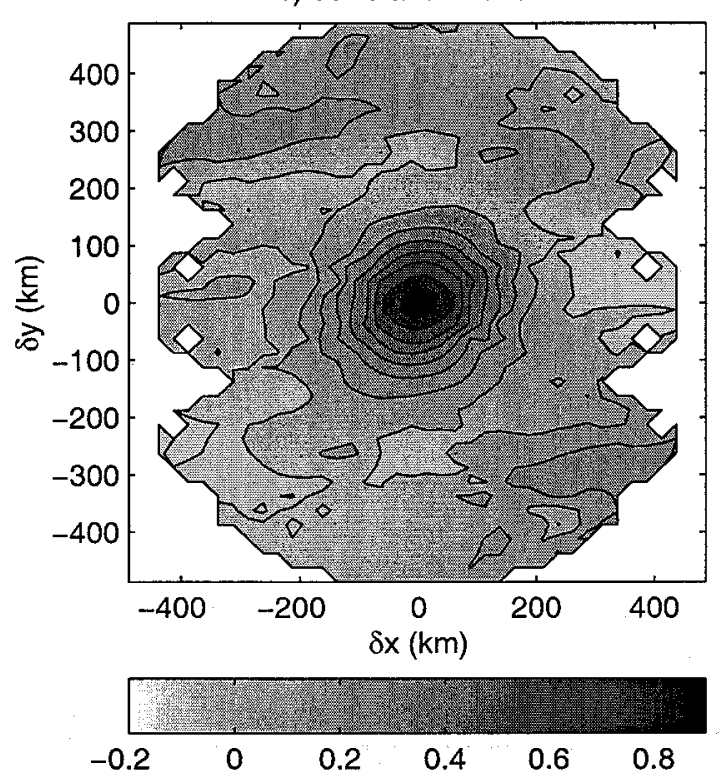

d) correlation zone $\mathrm{f} 4$

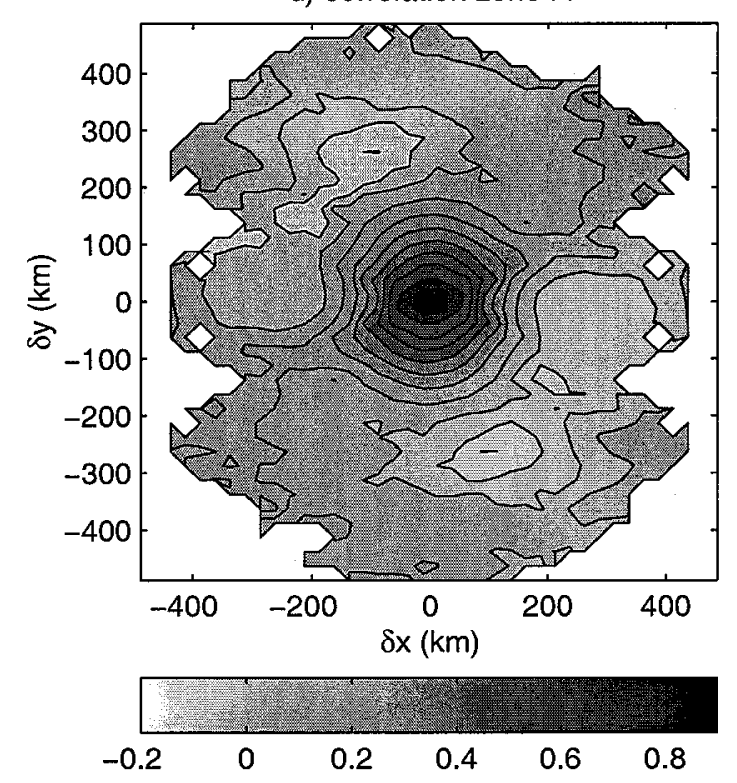

Figure 3-12: Same as Figure 3-11 but for the bins to the south of the Gulf Stream 
a) Confidence interval Zone g1
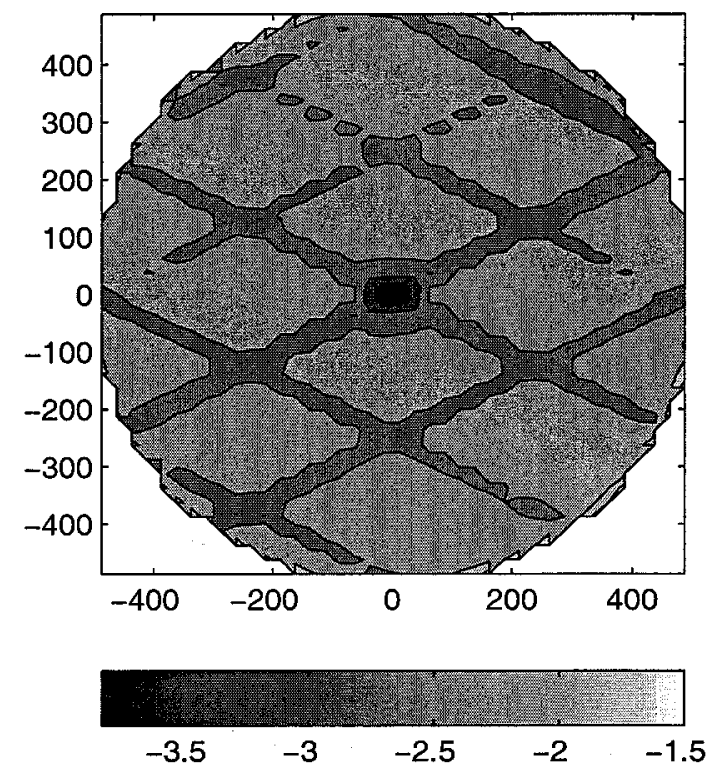

c) Confidence interval Zone g3

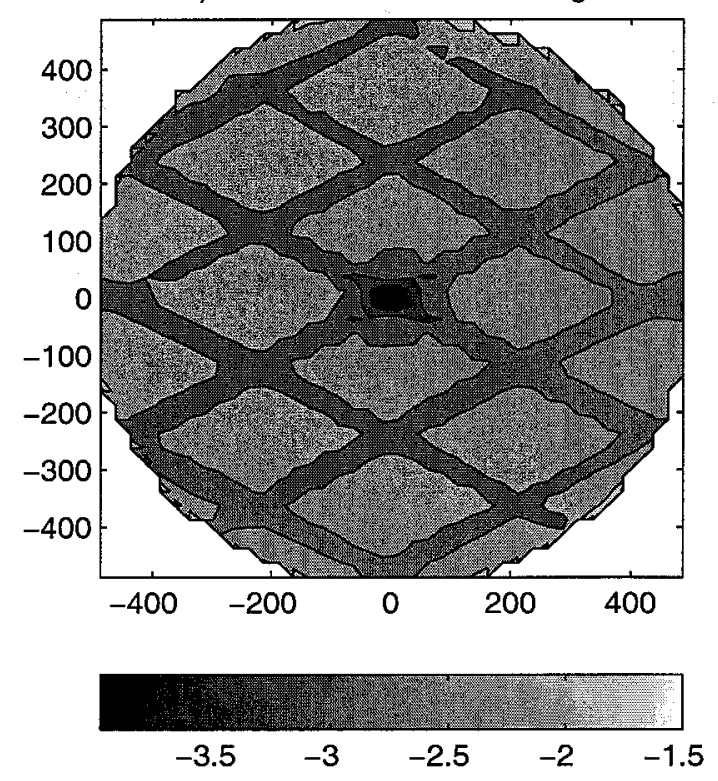

b) Confidence interval Zone g2
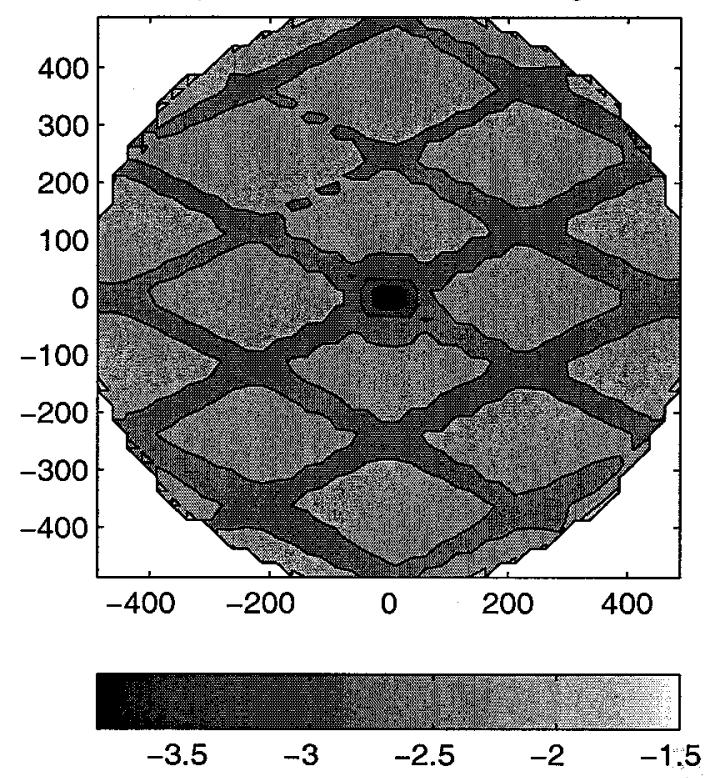

d) Confidence interval Zone g4

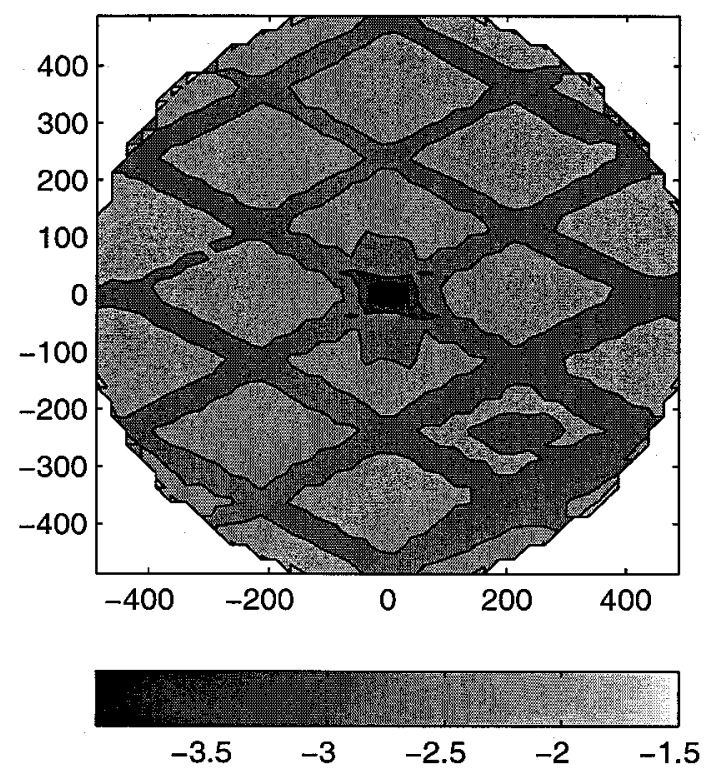

Figure 3-13: Confidence intervals in $\log _{10}$ for the sea surface height correlations along the Gulf Stream (Figure 3-11) 
a) Confidence interval Zone f1
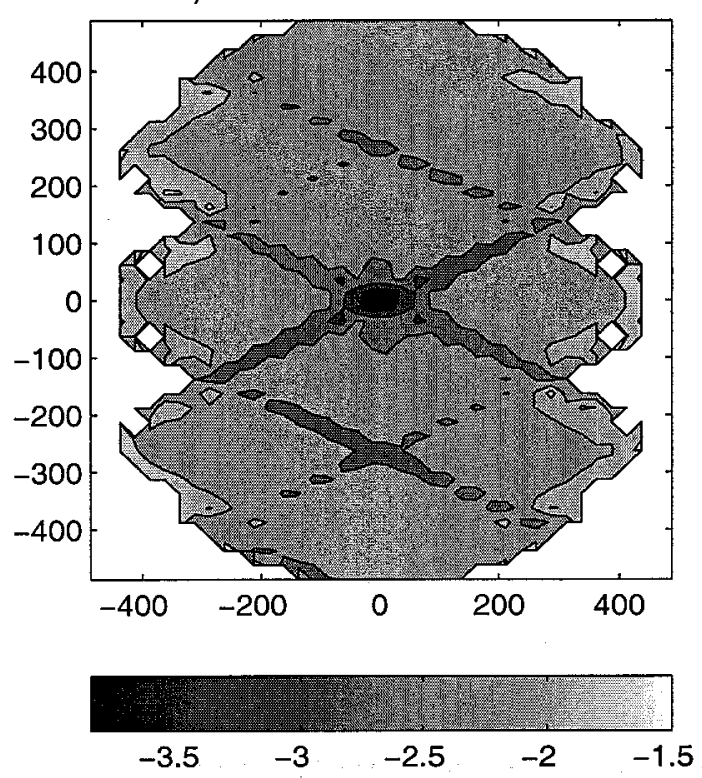

c) Confidence interval Zone $\mathrm{f3}$

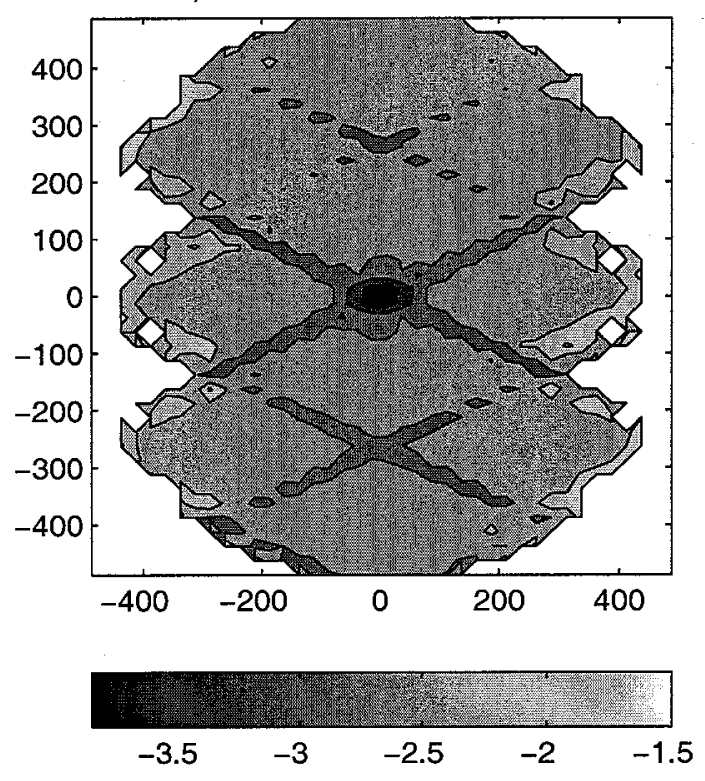

b) Confidence interval Zone $\mathrm{f} 2$
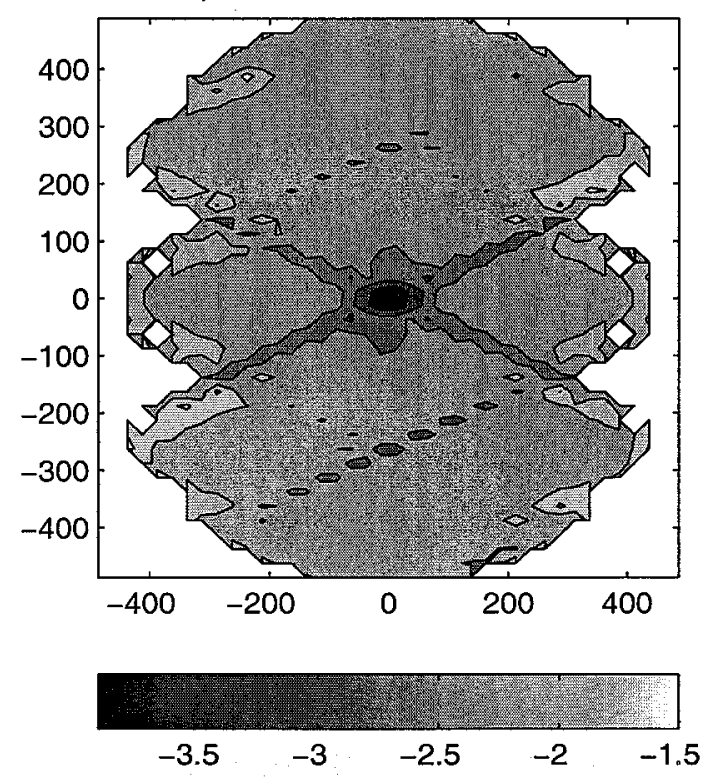

d) Confidence interval Zone f4

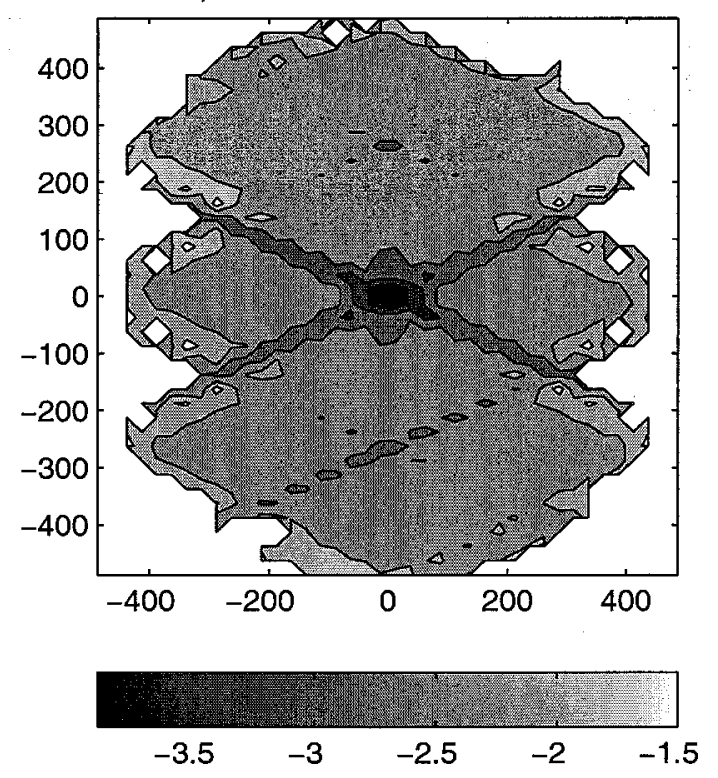

Figure 3-14: Confidence intervals in $\log _{10}$ for the sea surface height correlations to the south of the Gulf Stream (Figure 3-12) 
a) covariance zone g1

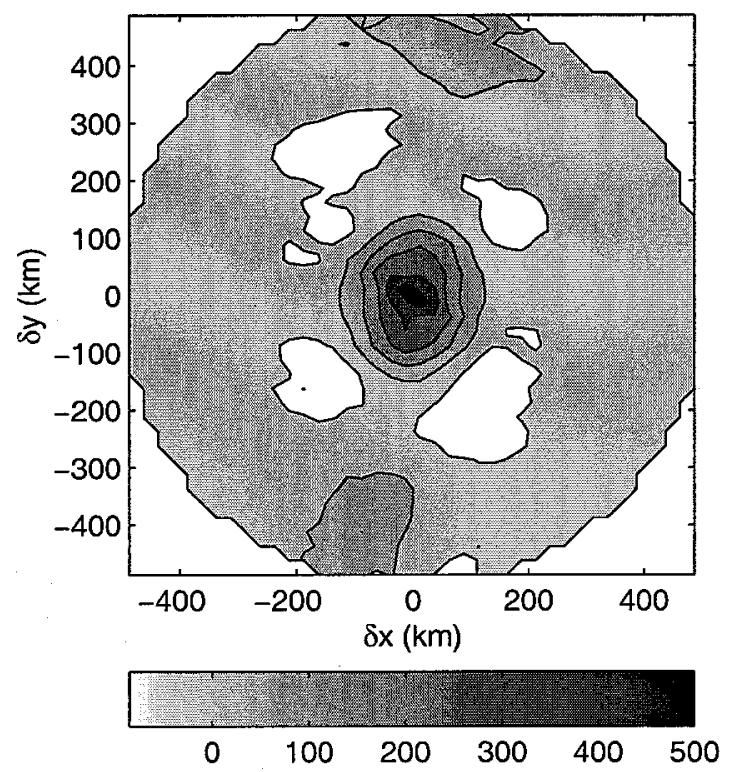

c) covariance zone g3

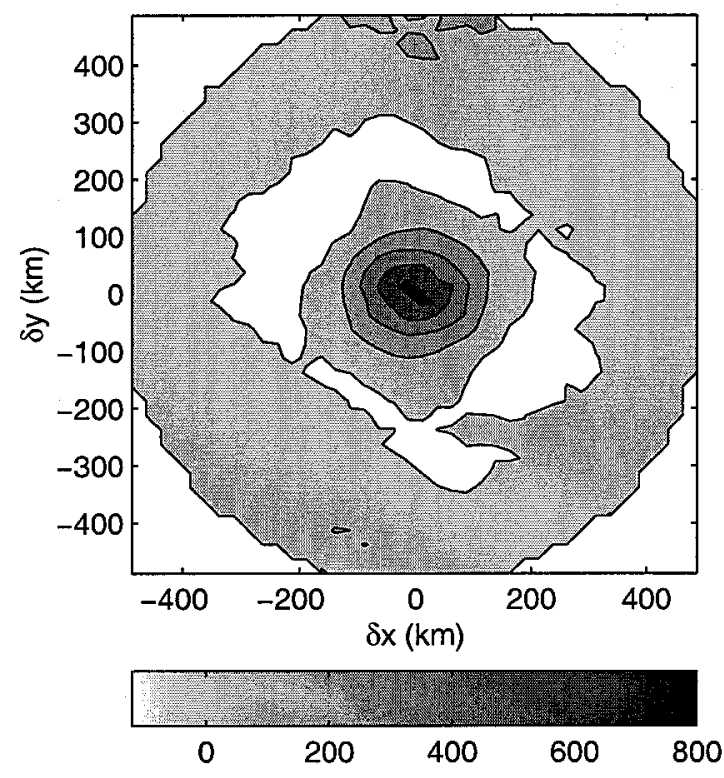

b) covariance zone g2

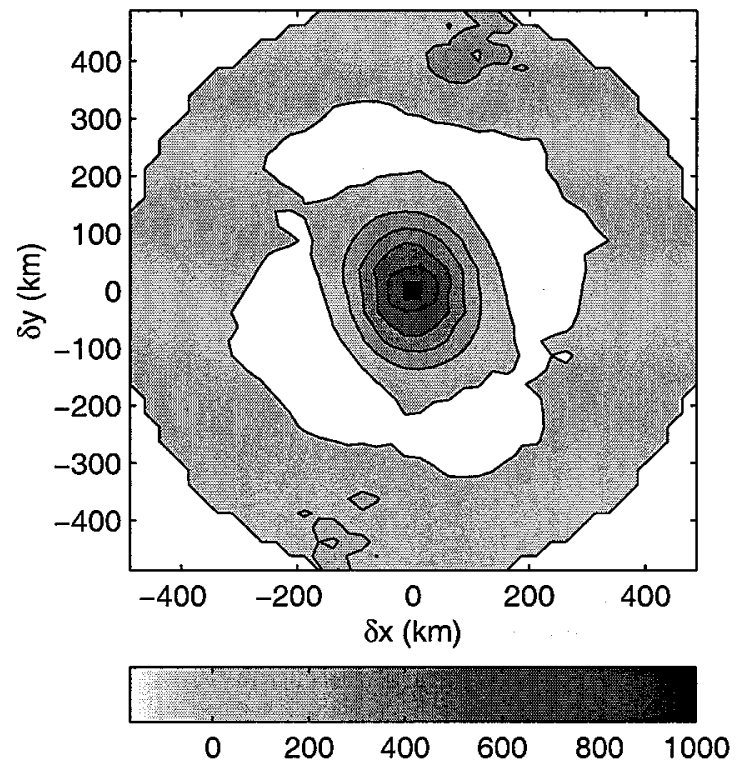

d) covariance zone g4

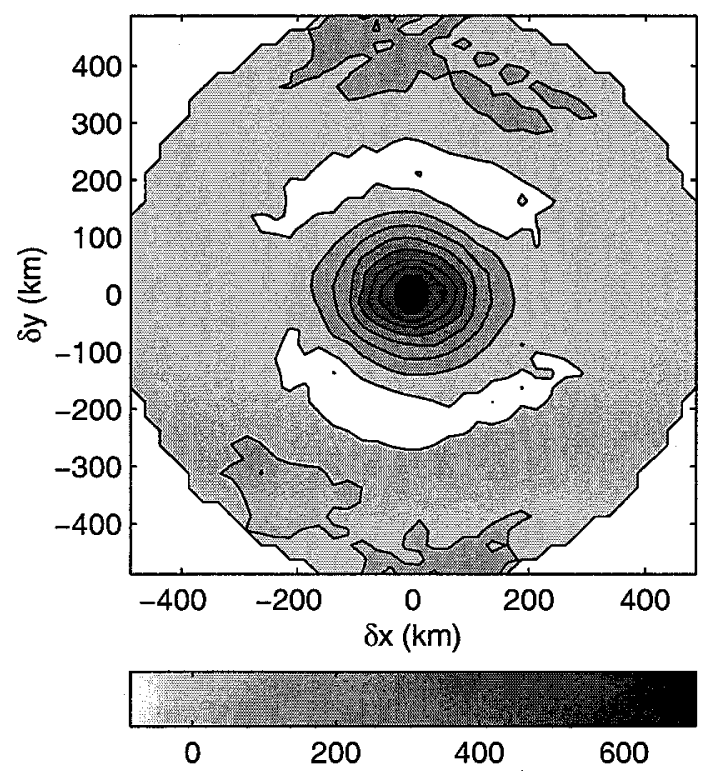

Figure 3-15: Two-dimensional covariance computed from TOPEX/Poseidon data for the $5^{\circ}$ by $5^{\circ}$ bins along the Gulf Stream. 
a) covariance zone $\mathrm{f1}$

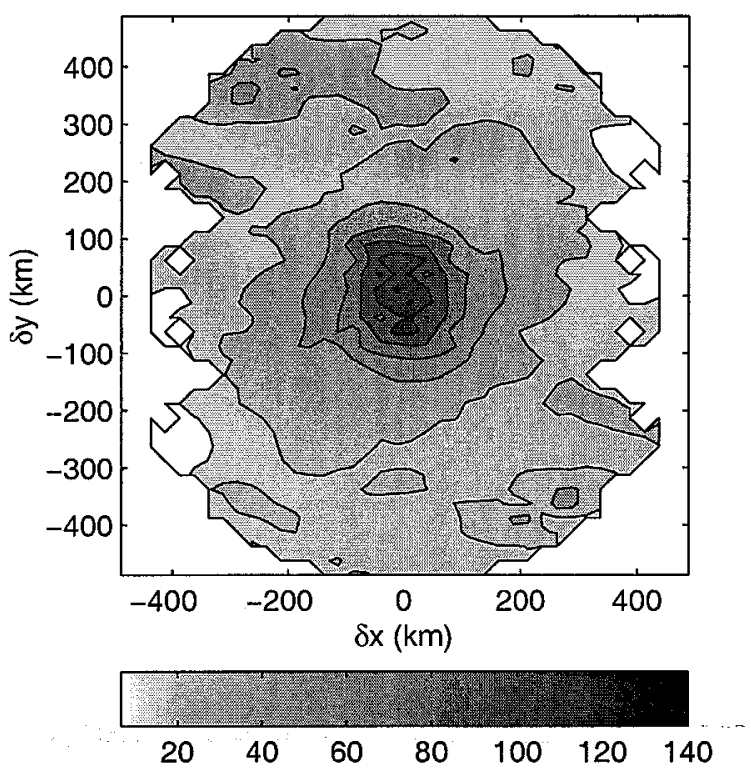

c) covariance zone $\$ 3$

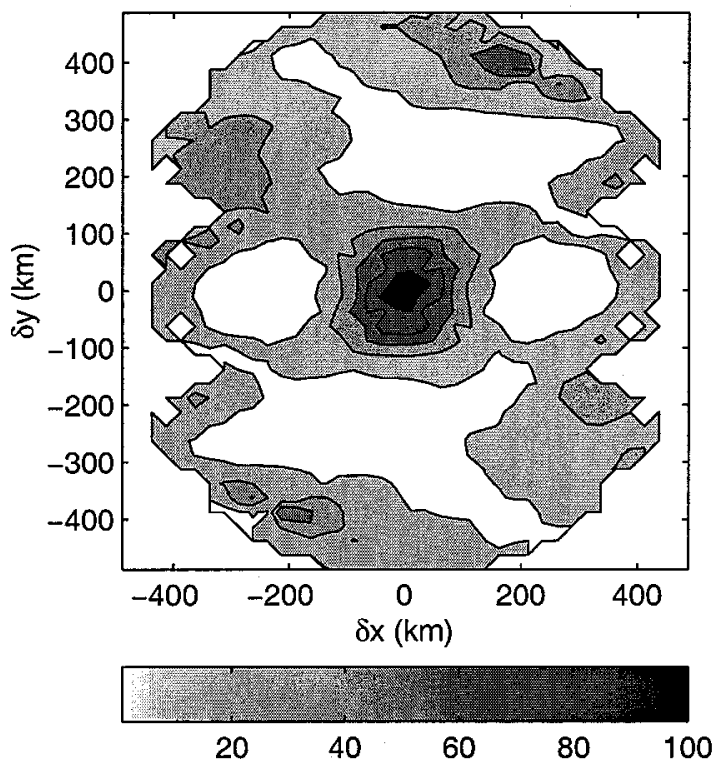

b) covariance zone f2
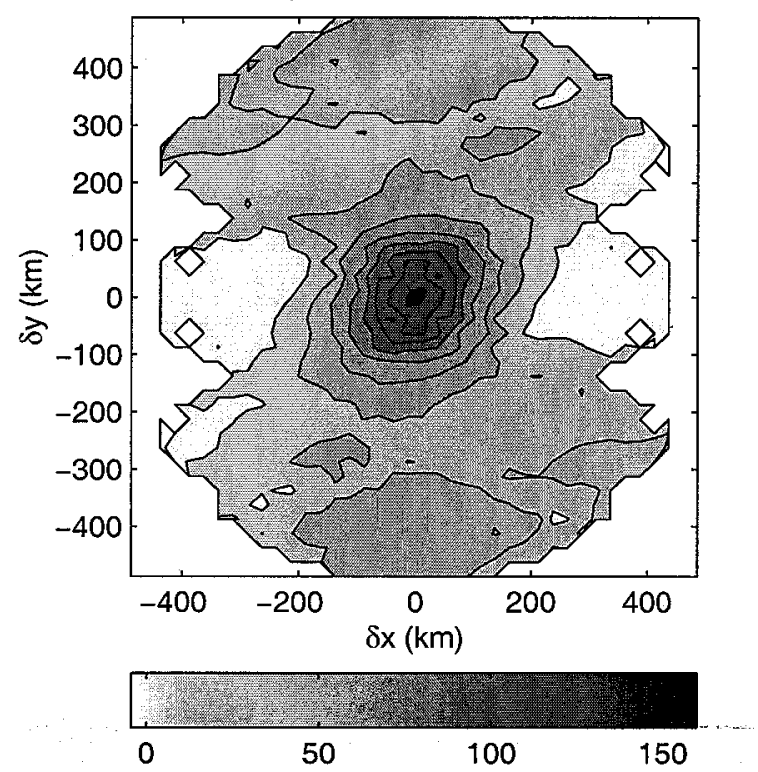

d) covariance zone f4

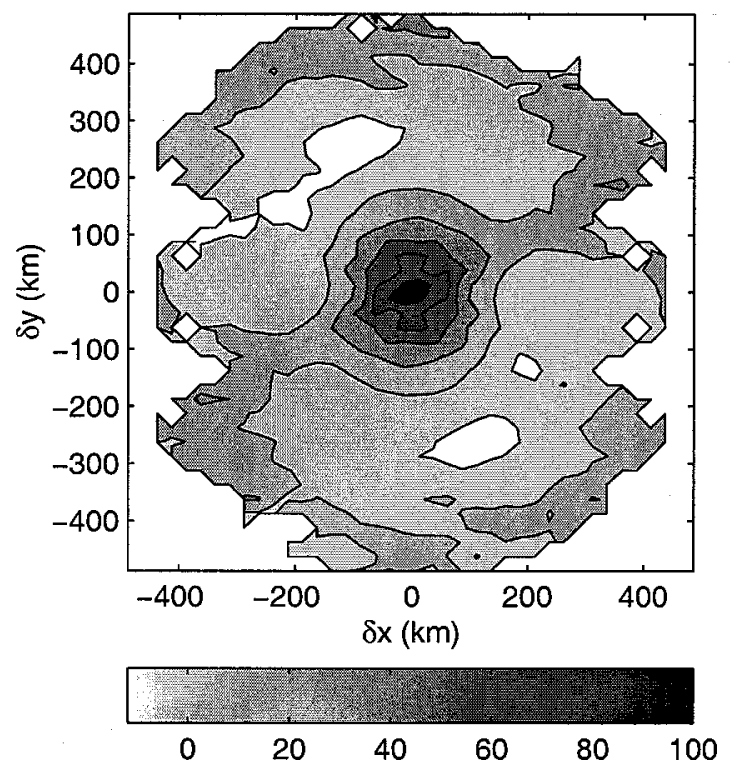

Figure 3-16: Same as Figure 3-16 but for the bins to the south of the Gulf Stream. 
assumes a student distribution. The null hypothesis is $C=0$, and it is tested for $95 \%$ in the following way. Compute,

$$
t_{s}=C \sqrt{(N-2) /\left(1-C^{2}\right)}
$$

and compare it with the student distribution value, $t_{0.95[N-2]}$. The null hypothesis is rejected when $t_{S}>t$. The student distribution does not have negative values; the negative of $t$ was used when testing for $t_{s}<0$. For many of the negative points the null hypothesis of $C=0$ was rejected. This result, and the persistence of the negative values suggest that they are not a product of a random process.

I suggest that the correlation can be modelled $\left(C_{M}\right)$ as the product of a gaussian and a cosine function,

$$
C_{M}=e^{-A_{1} r_{1}^{2}+A_{2} r_{2}^{2}+A_{3} r_{1} r_{2}} \cos \left(\sqrt{B_{1} r_{1}^{2}+B_{2} r_{2}^{2}+B_{3} r_{1} r_{2}}\right)
$$

The parameters of the equation can be found by fitting the data using a non-linear least-square method (MATLAB). Figure 3-17 shows the two-dimensional correlations of the sea surface height for the area $g 2$, the proposed correlation with the parameters found by the adjustment, $A_{1}=2.9 \times 10^{-11}, A_{2}=3 \times 10^{-11}, A_{3}=-0.3 \times 10^{-11}, B_{1}=$ $12 \times 10^{-11}, B_{2}=10 \times 10^{-11}$, and $B_{3}=3 \times 10^{-11}$. For the actual fitting, since the values of the correlation away from the center are centered around 0.1 and not zero, it was decided to fit the function: $c=0.1+0.9 e^{-A_{1} r_{1}^{2}+A_{2} r_{2}^{2}+A_{3} r_{1} r_{2}} \cos \left(\sqrt{B_{1} r_{1}^{2}+B_{2} r_{2}^{2}+B_{3} r_{1} r_{2}}\right)$. The correlation is near isotropic, since $A_{1} \simeq A_{2}, B_{1} \simeq B_{2}, A_{3} / A_{2}<1$ and $B_{3} / B_{2}<1$. The anisotropy is due mostly to the value of $B_{3}$ and the difference between $B_{i}$. This result will be used in section 5.2 when computing the eddy vorticity flux.

Glazman et al. (1996) suggested avoiding pairs of points that belong to the same transect to eliminate the influence of fast sea surface height oscillations caused by short-correlated processes, such as gravity waves, sea state bias oscillations (due to wind speed variations) etc. A comparison is made between the correlations computed with and without pairs of data that belong to the same transect to verify that by filtering the data there is no need to exclude same-transect point. Figure 3-18 shows 


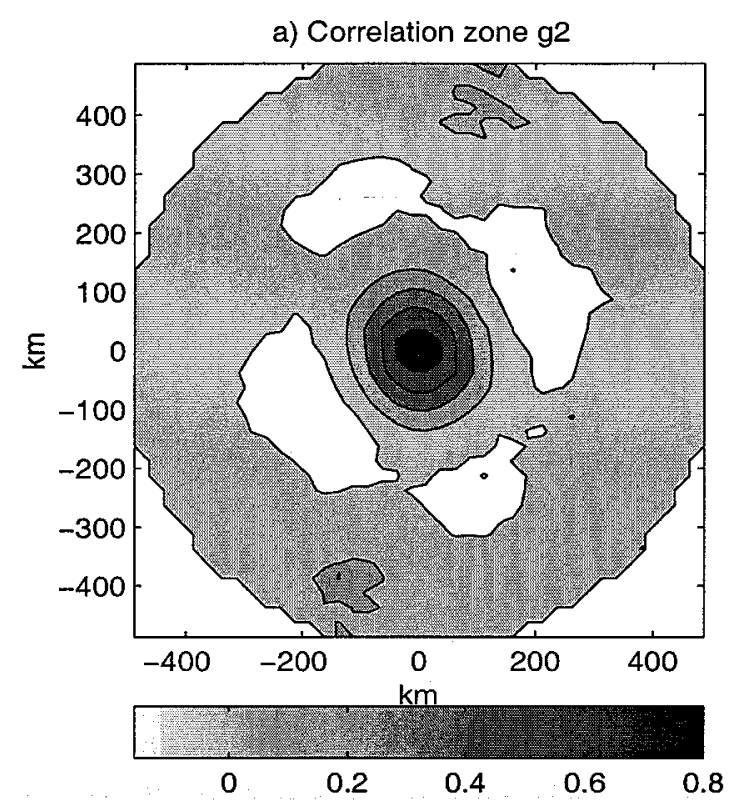

b) Proposed represenation for correlation

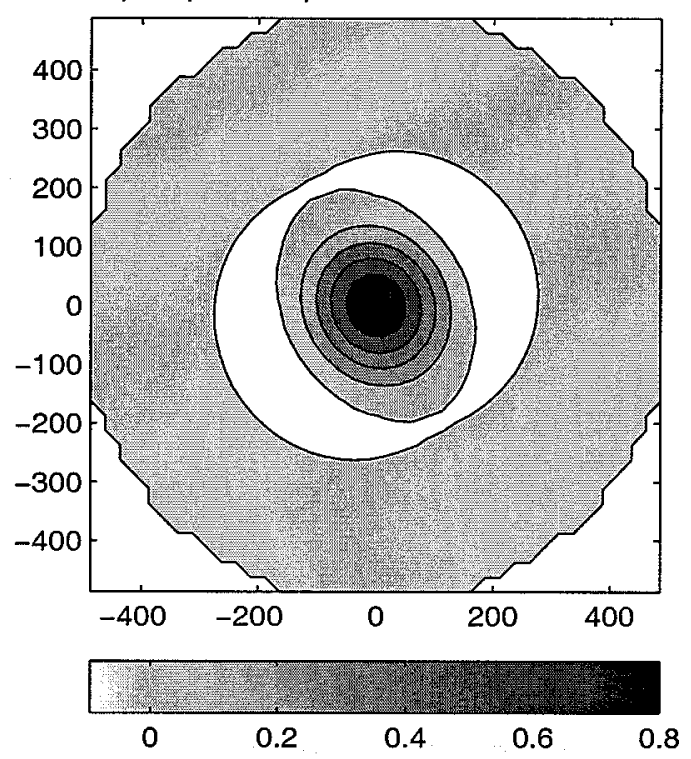

c) Residual of the least square fit.

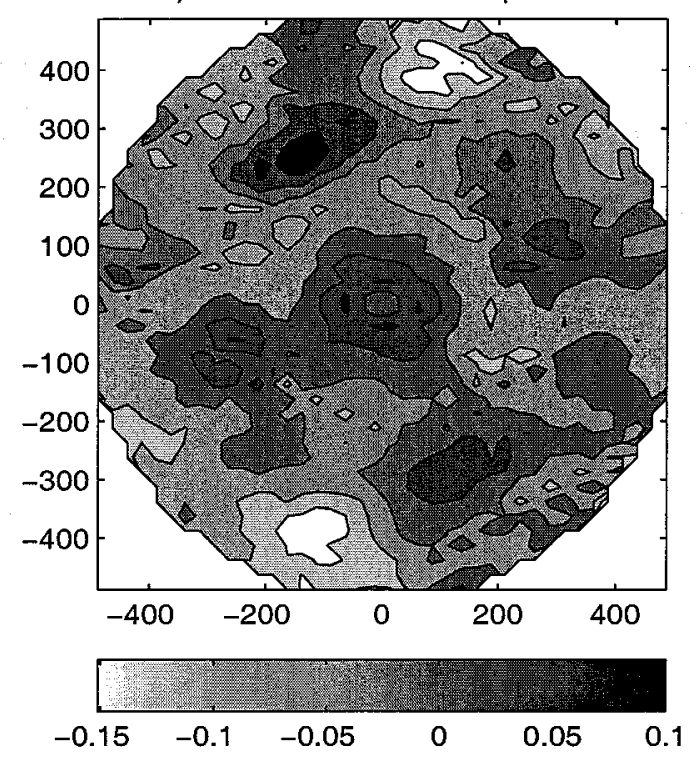

Figure 3-17: (a) Sea surface height correlation for a $5^{\circ}$ by $5^{\circ}$ area, (b) the proposed representation of the correlation as the product of a cosine times a gaussian function (see text) and (c) the residual of the least squares fit. 
the correlation for the zone $g 1$ when data of the same transect have been used (a) and when it was avoided (b). Few differences appear when pair of points that belong to the same transect are avoided, but they are small and no evidence of fast oscillations caused by short correlated processes are found. The differences can be explained by the reduction of the sample size to get an estimate, and to the elimination of the autocorrelation of points, which reduces the value in the center of the plots. The confidence intervals are plotted on panels (c) and (d). The differences are a manifestation of the reduction in degrees of freedom as a consequence of the change of available pair of points. The center of the plot in (c) has the smallest confidence interval as each point is autocorrelated. This autocorrelation is not made when points of the same transect are avoided.

The geometry of the arcs is present in both maps of the confidence intervals, and at certain degree, also in the correlation plots. The increase in sample size affects the correlation value. The inhomogeneity on the sample size might make it seem less isotropic due to the dependance of the correlation value in the sample size.

\subsection{Chapter Summary}

Processing of the satellite data are performed.

The exploration of two corrections for TOPEX/Poseidon data yields the result that the orbit error correction is not needed for TOPEX/Poseidon and that the barotropic correction does not affect the statistical analysis.

The sea surface correlation along the groundtracks, starting at the crossovers, suggest that the correlation does not follow a Gaussian distribution since it has negative values. Some correlation curves decline slowly, crossing through zero at large distances from the origin or not crossing at all. No explanation is found for these features.

The presence of negative values in the correlation of the sea surface height ob- 
a)

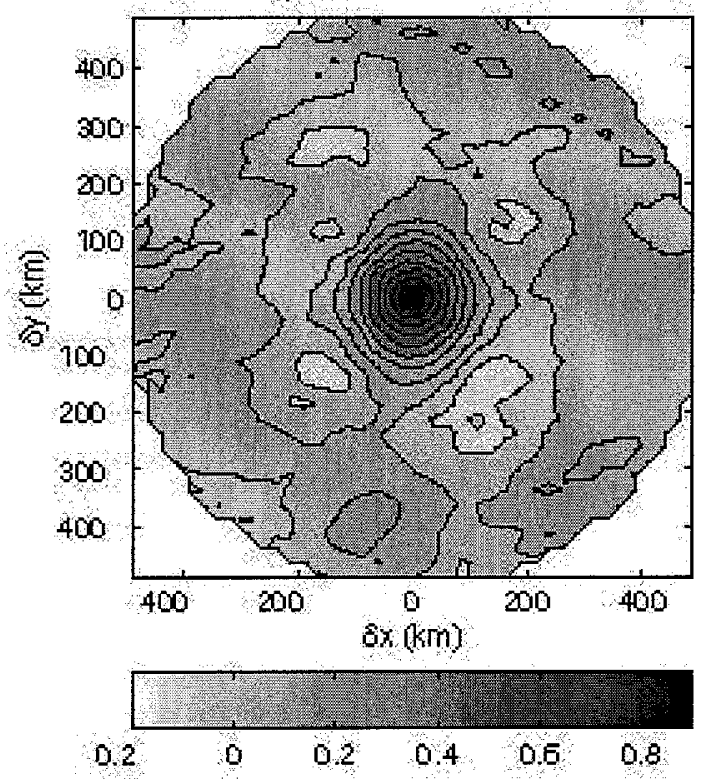

b)

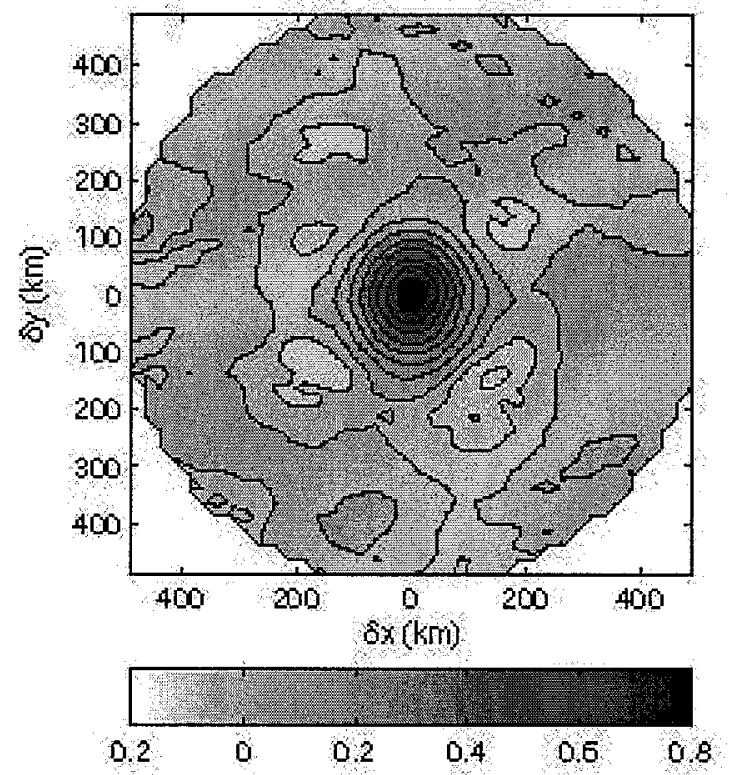

o)

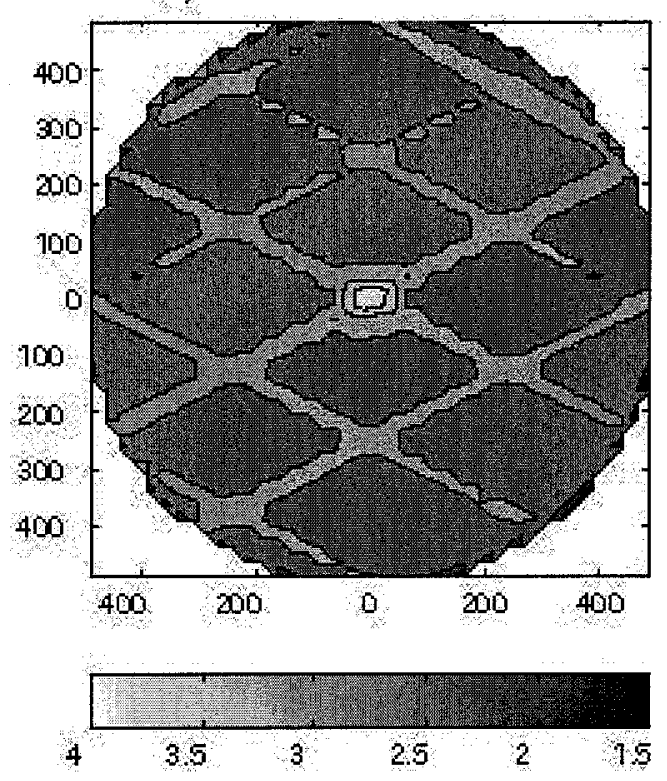

d)

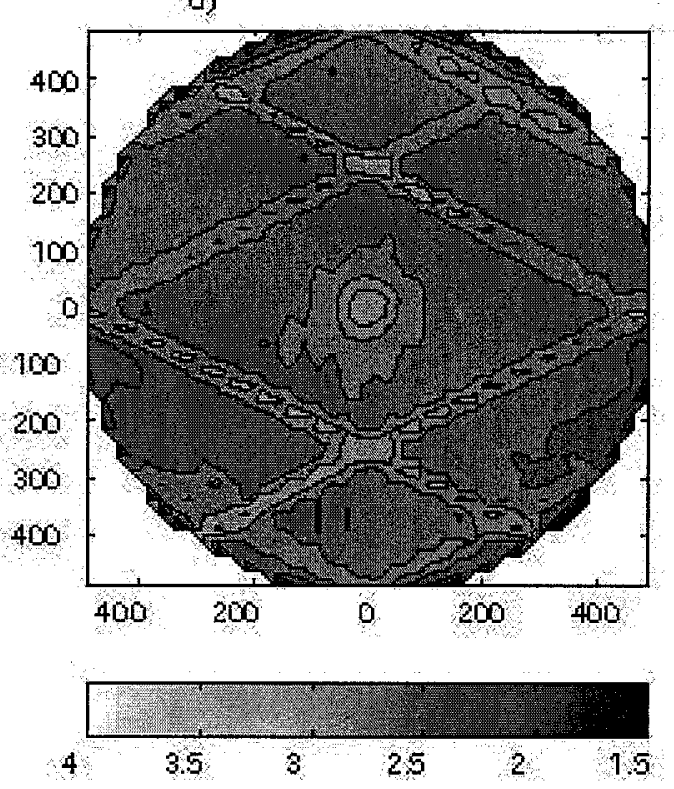

Figure 3-18: (Sea surface height correlation when point along the same transect have been included (a), and when they have been avoided (b). b) Logarithm of the confidence intervals for a), and d) logarithm of confidence intervals when same-arc points are not used. 
tained between the transects that cross $5^{\circ}$ by $5^{\circ}$ selected areas, placed along the Gulf Stream and to the south of it, confirm that the sea surface correlation does not follow a Gaussian distribution. A mathematical expression is found for modelling the correlation as the product of a gaussian times a cosine (Equation 3.16), which will be used in chapter 5 . The parameters of the mathematical approximation are found by a non-linear least squares fit (MATLAB). The correlations are found to be roughly homogeneous and nearly isotropic. 


\section{Chapter 4}

\section{Numerical Model}

Results from an eddy resolving numerical model are compared to the TOPEX/Poseidon data and used to evaluate the consistency of the methods employed in the analysis of the satellite data. The simulation is helpful as it provides a full view of a synthetic ocean that can be sampled as if a satellite had crossed above it. The sampled data provide a limited view of the full numerical rendition of the ocean just as TOPEX/Poseidon supplies a constrained view of the real ocean. We can learn more about the information that TOPEX/Poseidon gives by comparing the results of the analysis of the full model data to the ones from the sampled data.

A subdomain of the North Atlantic numerical simulation from the POP model at $0.1^{\circ}$ of resolution was provided by Dr. Jayne and Dr. Bryan of the National Center for Atmospheric Research (NCAR). It extends from $-75^{\circ}$ to $-50^{\circ}$ longitude and $30^{\circ}$ to $45^{\circ}$ latitude and has a repeat period of 10 days.

Smith et al. (2000) describe similarities and differences between the altimetric data and the simulation. Some of the features described in their work will be mentioned here, as well as new information provided by this work. As Smith et al. (2000) noted, the Gulf stream flows more zonally after it separates fris suggested by observations, resulting in a southward displacement in the mean path of the jet of $1^{\circ}$ to $1.5^{\circ}$ latitude. This can be seen in the maps of sea surface height variance: TOPEX/Posedion's in 


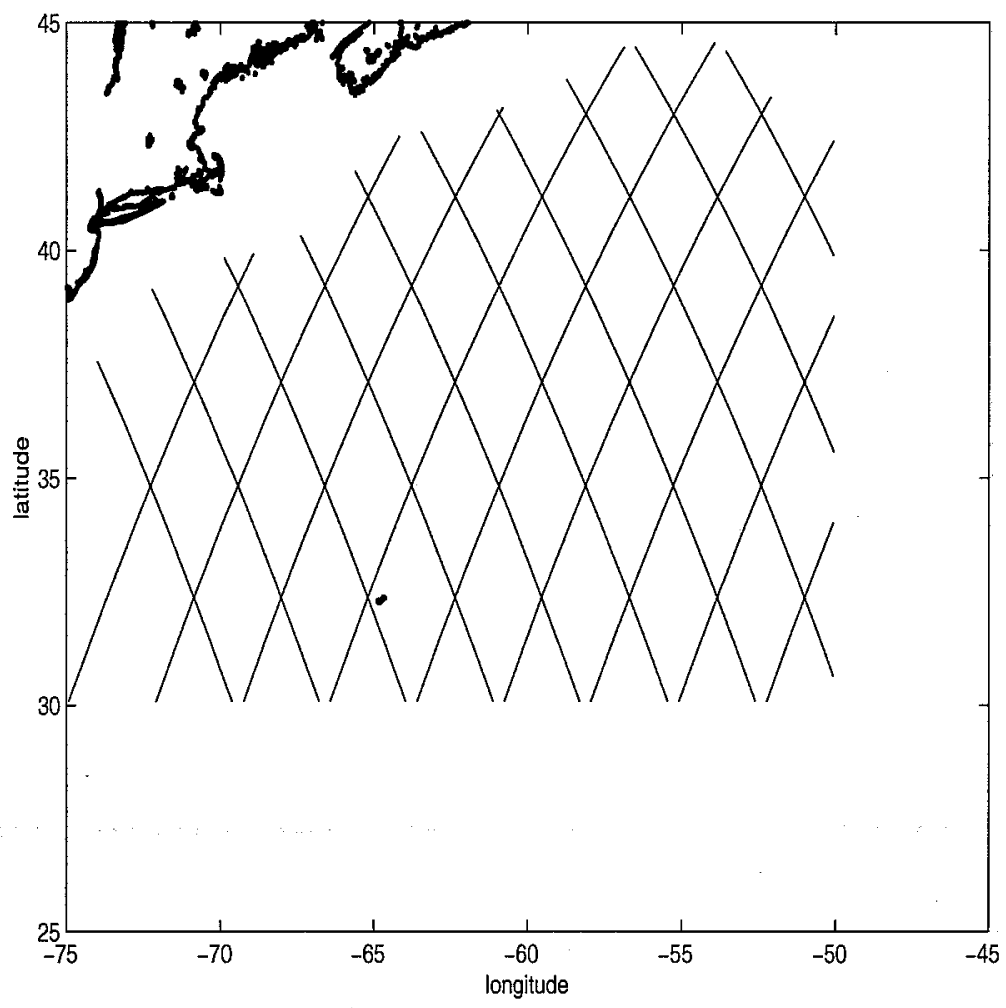

Figure 4-1: TOPEX/Poseidon transects employed to sample the numerical simulation data.

Figure 3-10 and the model's in Figure 4-6. The maps share the order of magnitude of the variability, but the TOPEX/Poseidon result is centered at a higher latitude. Both data sets show a widening of the variability field between $-65^{\circ}$ and $-60^{\circ}$ of longitude, to the east of the New England Seamounts; the numerical model has a wider envelope.

The numerical simulation was sampled along the TOPEX/Poseidon groundtracks (Figure 4-1). Since the model data has a repeat period of 10 days, all the model data sampled along the satellite groundtracks for a time repetition are simultaneous in time. This data set will be called POX data, while the term "model data" is reserved for the gridded one. 


\subsection{Velocity Correlations}

The eddy kinetic energy field obtained from the simulation's surface velocity field is compared to the eddy kinetic energy field inferred from the model's sea surface height at the cross overs (Figure 4-2, a and b). The POX map has less structure than the full model data and presents smaller values than the full model data. This is due to the loss of resolution by using velocity values only at the cross overs. Crossovers in the patches of high values would be needed to recover these features, but these patches are smaller than the crossover resolution. Nevertheless, the general shape and magnitude of the eddy kinetic energy is similar. This comparison gives confidence that the eddy kinetic energy obtained from TOPEX/Poseidon data represents the broad features of the real ocean's eddy kinetic energy (Figure 3-2 and plotted again in 4-2 to facilitate the comparison).

The TOPEX/Poseidon eddy kinetic energy has higher values than both renditions of the numerical model. The high values are found farther to the east in the satellite data than the numerical model. Also, the TOPEX/Poseidon map has the higher values in three patches probably as a result of poor resolution given by the cross overs.

The variance ellipses for POX data are computed as described in section 3.2, but are normalized by the maximum semimajor axis of the TOPEX/Poseidon ellipses in order to compare their magnitude. Figure 3-3 shows the POX ellipses in solid lines, and the TOPEX/Poseidon ellipses in broken lines to facilitate the comparison. The variability in the model is more isotropic and has a smaller magnitude than the variability captured by the satellite. It is again evident that the area with more variability in the TOPEX/Poseidon data is to the north from the high variability area of the model. In both cases, there is evidence that the velocity is not isotropic along the Gulf stream.

The low resolution obtained by the crossovers suggest that it might be better to use all the satellite data to map the eddy kinetic energy, as did Stammer (1997) 
a) Numerical simulation

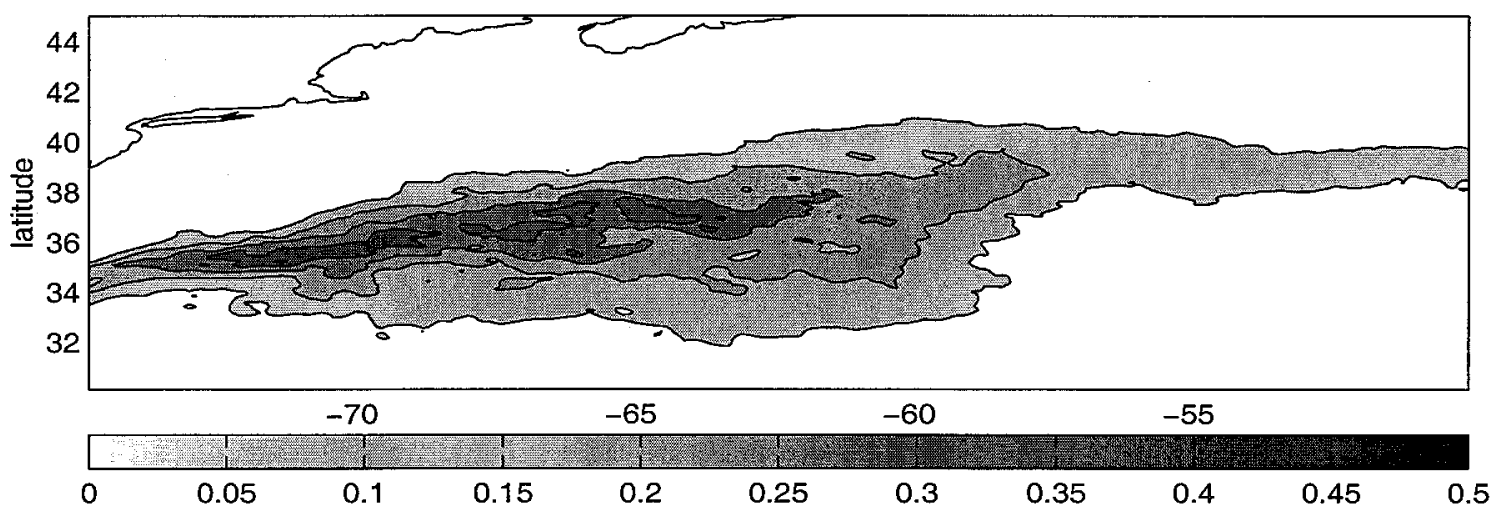

b) Numerical simulation at TOPEX/Poseidon crossovers

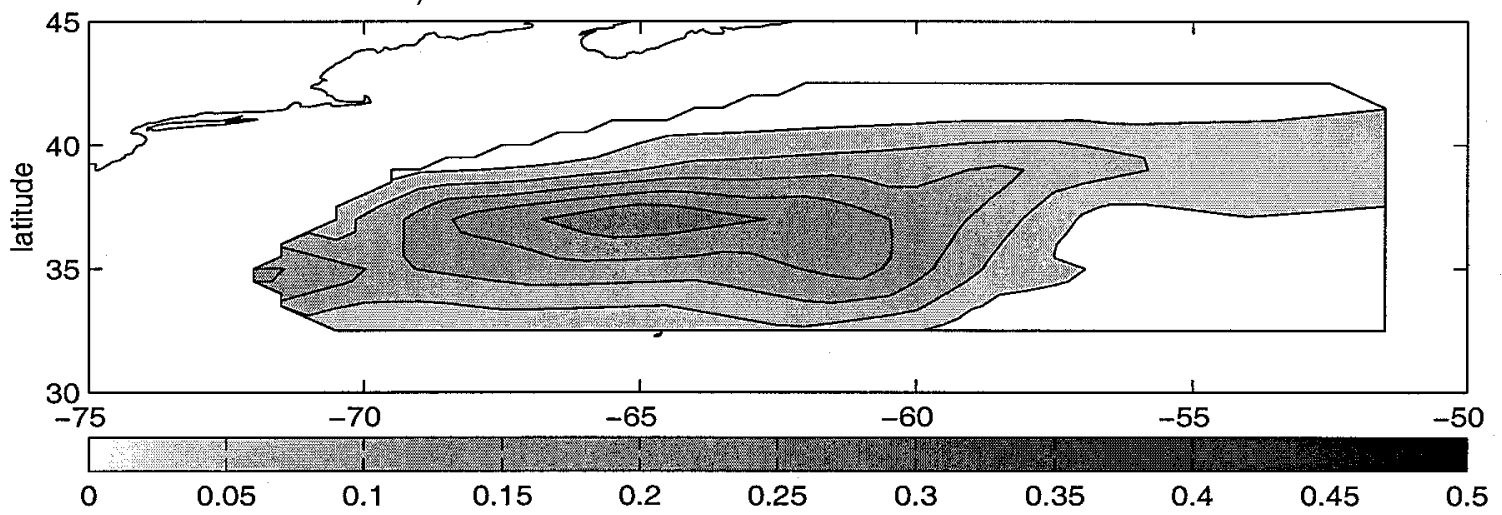

c) TOPEX/Poseidon's eddy kinetic energy

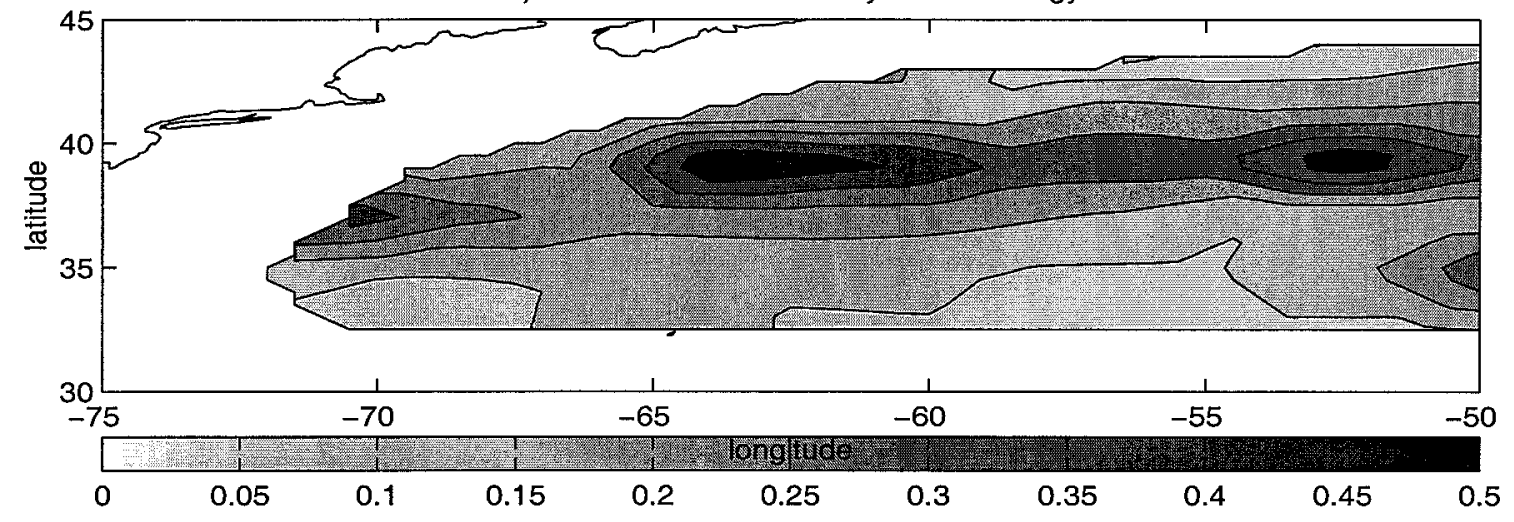

Figure 4-2: Eddy kinetic energy in $m^{2} s^{-2}$ computed from (a) surface velocity of the numerical simulation, (b) geostrophic velocity derived from POX sea surface height, and (c) geostrophic velocity derived from TOPEX/Poseidon sea surface height data. 


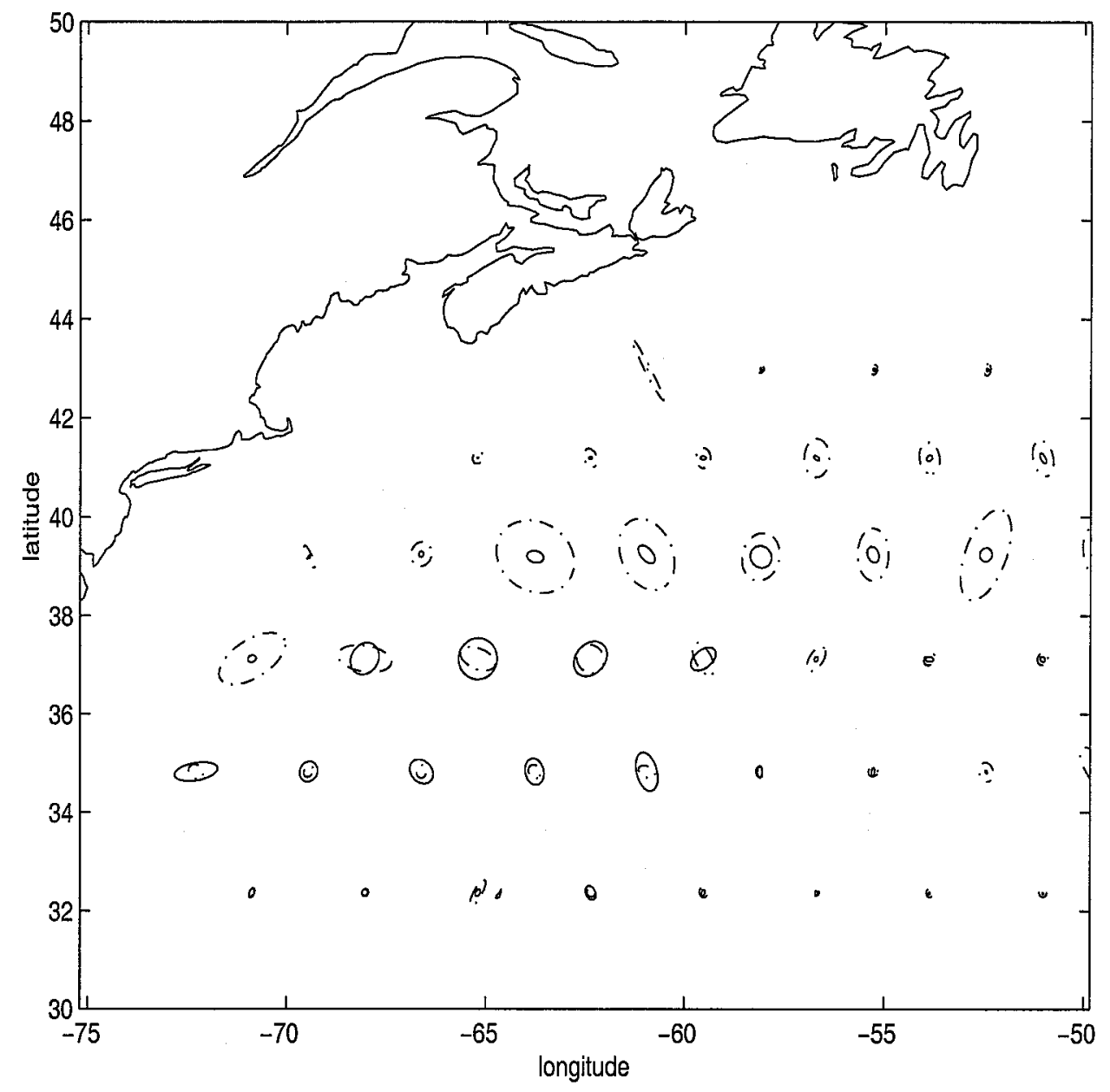

Figure 4-3: Variance ellipses for the model data sampled as TOPEX/Poseidon (solid line) and for TOPEX/Poseidon data (dashdot line)

assuming the velocity is isotropic, but the variance ellipses show that the isotropic assumption is not satisfied by the velocity field.

\subsection{Along Track Correlations}

Along track correlations for the sea surface height are computed on the POX data in the same way they were computed in the previous chapter for the TOPEX/Poseidon data (section 3.3). The time series at the crossover of two arcs is correlated with the time series at different positions along the same arc (equations 3.8 and 3.9). The 
correlation thus obtained is a function of the distance from the cross over.

Most of the curves of the correlations have a similar behavior. They decline to zero, dip into negative values and then go back to the positive side to finish oscillating around zero. Some curves reach further into the negative values than others, and most go back to the positive side and remain near zero or stay below the critical correlation value below which the values are not statistically significant from zero. Figure (4-4) is a good example of this behavior. It corresponds to the along track correlation at the crossover formed by arcs 176 and 191 (-56.6 $6^{\circ}$ lon, 37.11 lat). Panel (a) shows the correlations for arc 176, South-East direction (solid line) and North-West (dash-dot line) are shown. In (b), the correlations for arc 191, North-East (solid line) and SouthWest (dash-dot line). The dotted line indicates the smallest value that is significantly different from zero (Sokal and Rohlf, 1981)

The correlation of the POX data does not show curves that decay slowly as the altimeter data did (Section 3.3). They do present, on the other hand, high correlation values at large distances $(>200 \mathrm{~km}$ ) from the cross over. Figure $4-5$ shows the crossover with the highest correlation value at more than $200 \mathrm{~km}$ from the cross over. It corresponds to the crossing of the transects 74 and $115\left(-55.2^{\circ}\right.$ lon and $42.97^{\circ}$ lat). The correlation reaches 0.35 in the North-East direction (solid line in (a), arc 74) at $291 \mathrm{~m}$ from the crossover, and 0.43 at $494 \mathrm{~km}$ in the South-West direction (dashdot line in (b), arc 115). The dotted line is at the smallest value that is significantly different from zero ( 0.2 in this case). It is not clear if this corresponds to a tapered periodic behavior (which would suggest the presence of long waves) or a spurious feature created by the simulation, or an outlier from a random process.

\subsection{Two Dimensional Correlations}

Two dimensional sea surface height correlations were computed for the TOPEX/Poseidon data in section (3.4). The same computation is made for the model data as well as for 

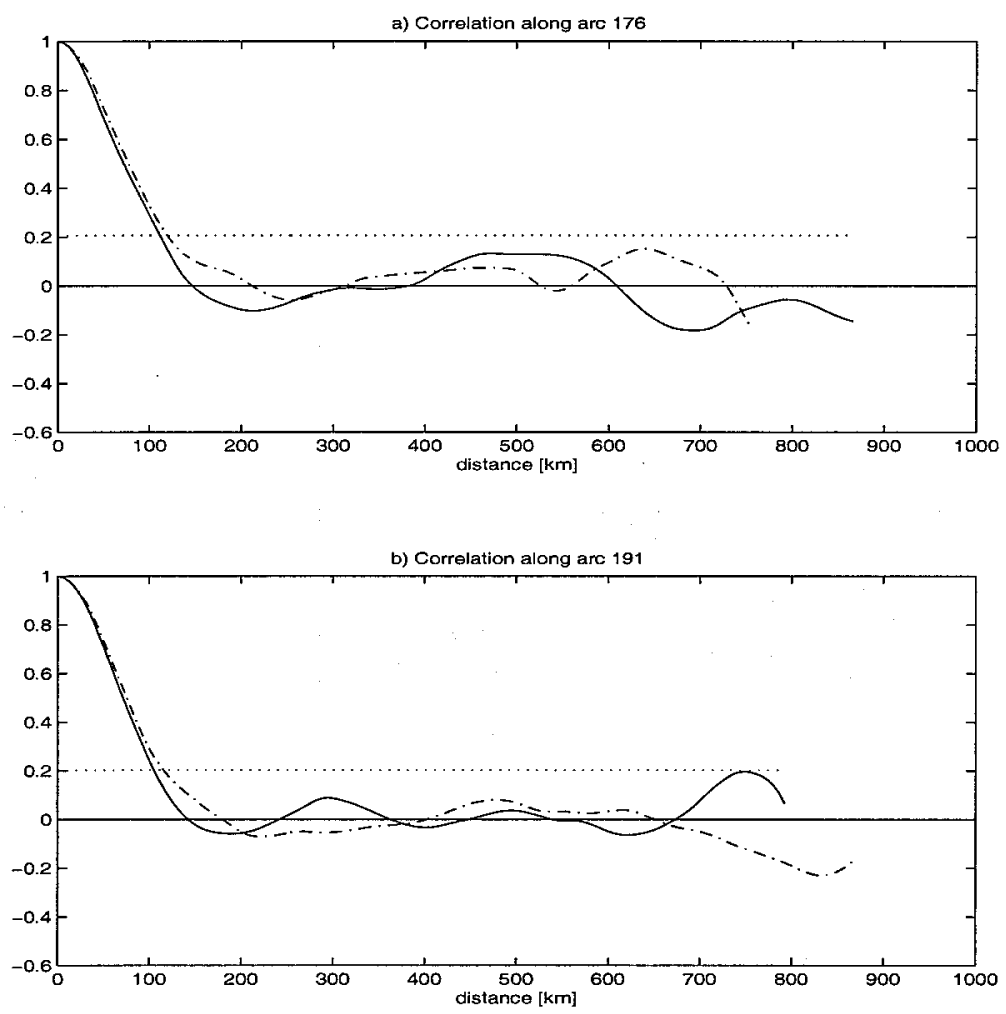

Figure 4-4: Along track correlation for the numerical simulation sampled along TOPEX/Poseidon transects, at the cross over formed by arcs 176 and $191\left(-56.6^{\circ} \mathrm{lon}\right.$, $37.11^{\circ}$ lat). Directions (a) South-East (solid lines) and North-West (dash-dot line) for arc 176, and (b) North-East (solid line) and South-West (dash-dot line) for arc 191. Dotted line at the smallest value significantly different from zero 

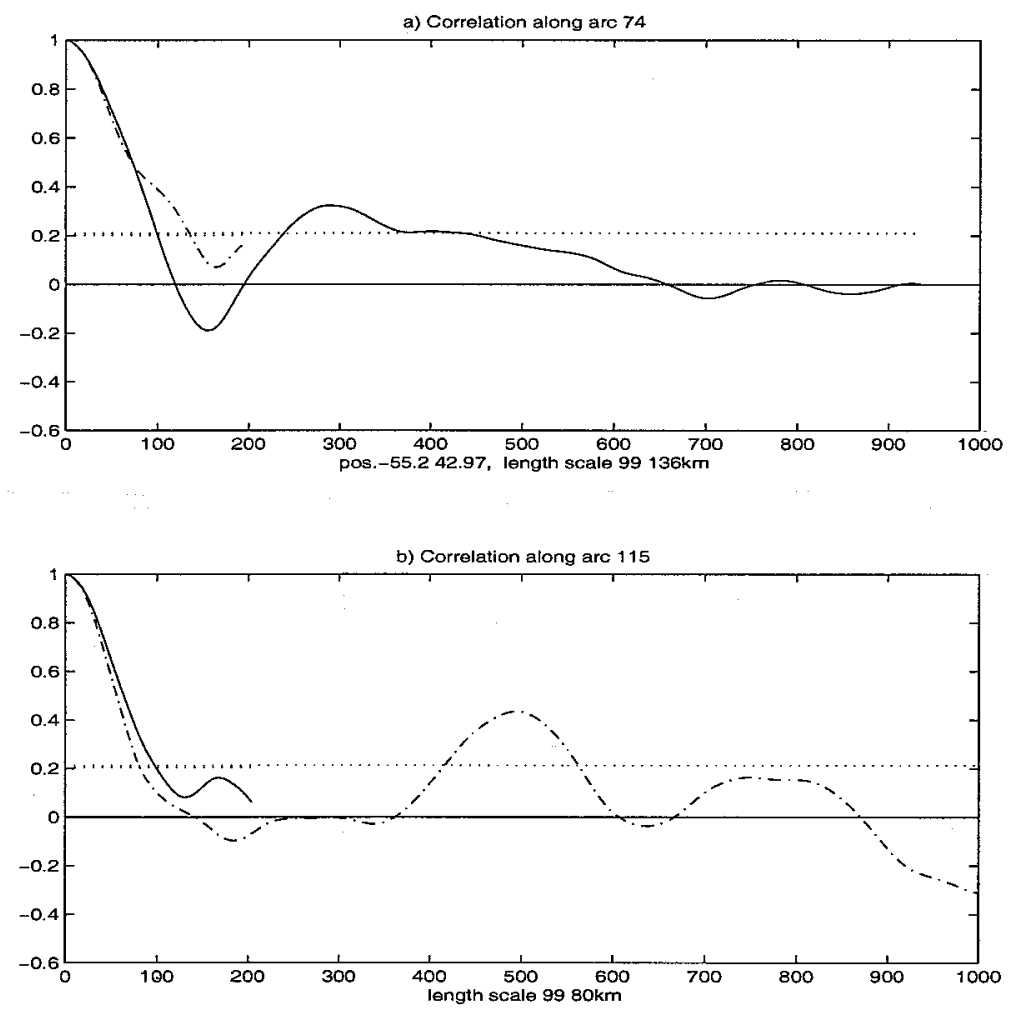

Figure 4-5: Along track correlation for the numerical model data sampled as TOPEX/P where high correlation values appear at distance from the cross over. Crossover by arcs 74 and $115\left(-55.2^{\circ}\right.$ lon and $42.97^{\circ}$ lat $)$. Directions a) South-East (solid lines) and North-West (broken line) for arc 74, and b) North-East (solid line) and South-West (broken line) for arc 115. Dotted line at the smallest value significantly different from zero. 
the model data sampled along the satellite's groundtracks (POX data). A comparison can be made between the correlations obtained using all the numerical data and those using the POX data to see how much information is lost in the altimetry sampling. Another comparison can be made among TOPEX/Poseidon and POX correlations.

Two dimensional correlations were obtained using different arcs in the same way they were computed for the altimetry data (section 3.4 ). The $5^{\circ}$ by $5^{\circ}$ areas used in this case are shown in Figure 4-6. The variance of the sea surface height is also displayed to show the position of the areas with respect to the Gulf Stream. The model domain available is not as extensive as the region of the satellite data and most of the model's domain shows large variability corresponding to Gulf stream influence. Only one area can be placed outside the current (area f1), while the three positioned along the current were overlapped, as done by Glazman et al. (1996) in order to fit more areas.

It can be seen in Figures 4-7 and 4-8 that the two dimensional covariance does not change significatively with the POX sampling. The model covariance has softer lines and the curves appear more circular. The POX covariances, on the other hand, have harder edges and seem to be more rectangular. The position of the data points explains these small differences.

The same comparisons are true for the correlation. Figures 4-9 and 4-10 show that the curves for the model appear more circular than the POX ones.

The $\log _{10}$ of the confidence intervals for the correlation of the POX data are shown in Figure 4-11 (Sokal and Rohlf, 1981). The difference between the upper and lower confidence interval is very small, so one plot is presented for both cases. Note that the confidence intervals are quite small, from $5 \times 10^{-2}$ to $1 \times 10^{-4}$. The range covers a couple of orders of magnitude, so the logarithmic scale is used to preserve the structure.

The covariance and correlation do not follow a gaussian distribution. Like the TOPEX/Poseidon curves, they have negative values at some distance from the origin. The model curves have smaller values (more negative) than the TOPEX ones. 


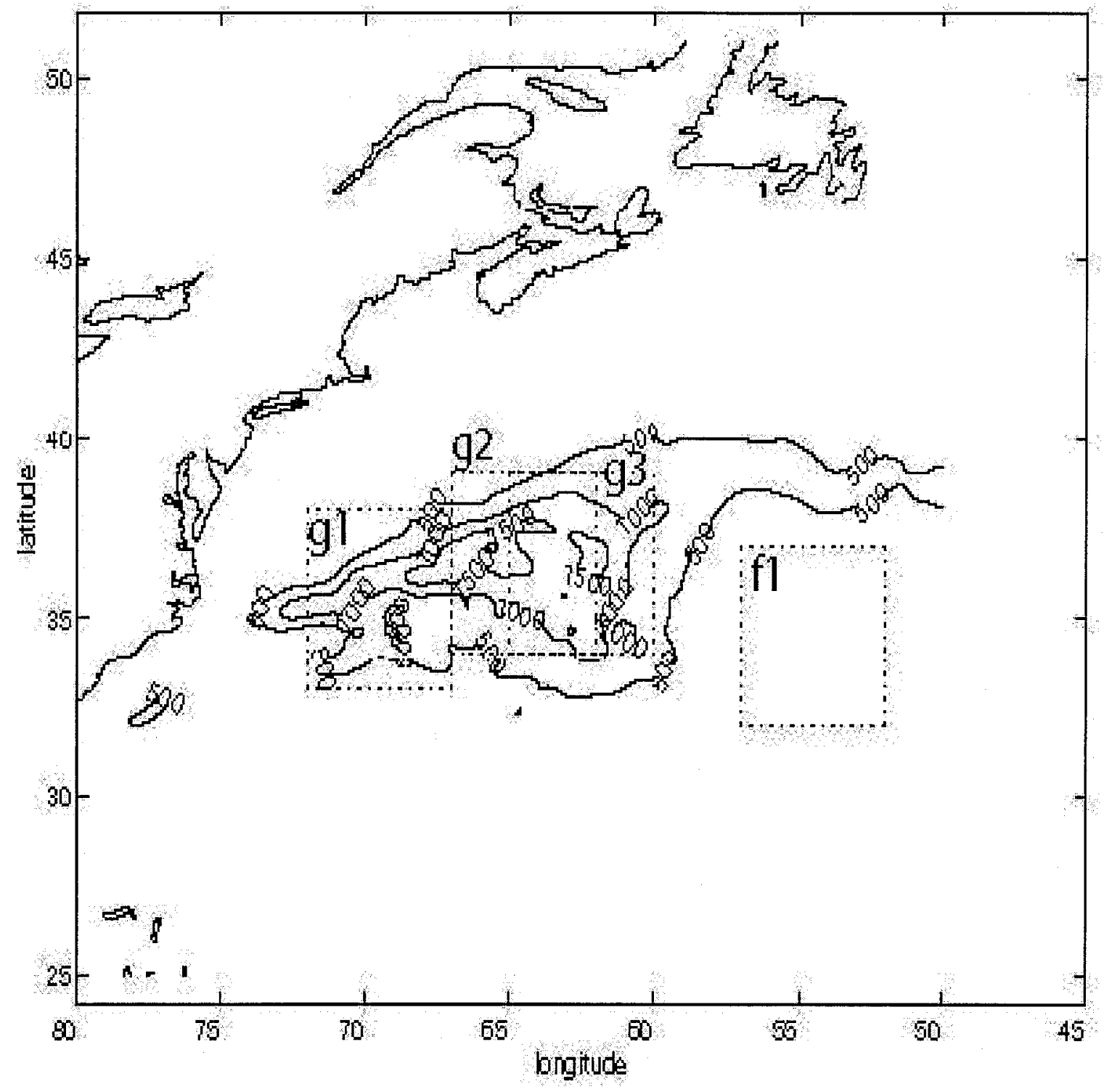

Figure 4-6: Sea surface height variance from the POP model. Dotted lines show the areas selected for computing the 2-dimensional correlations The Gulf stream influence is identified by the high variability values. 

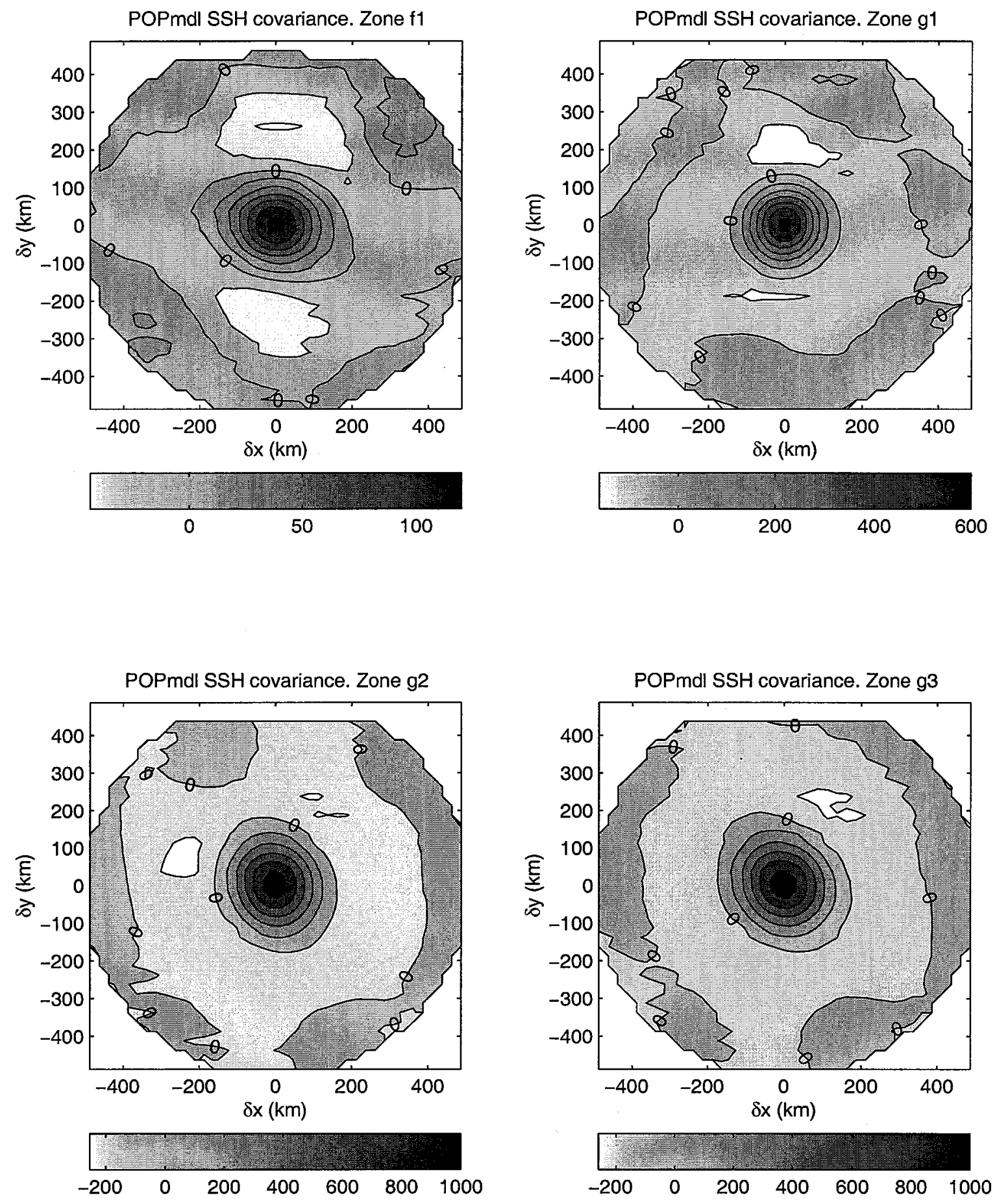

Figure 4-7: Two dimensional covariance using all the numerical model data 

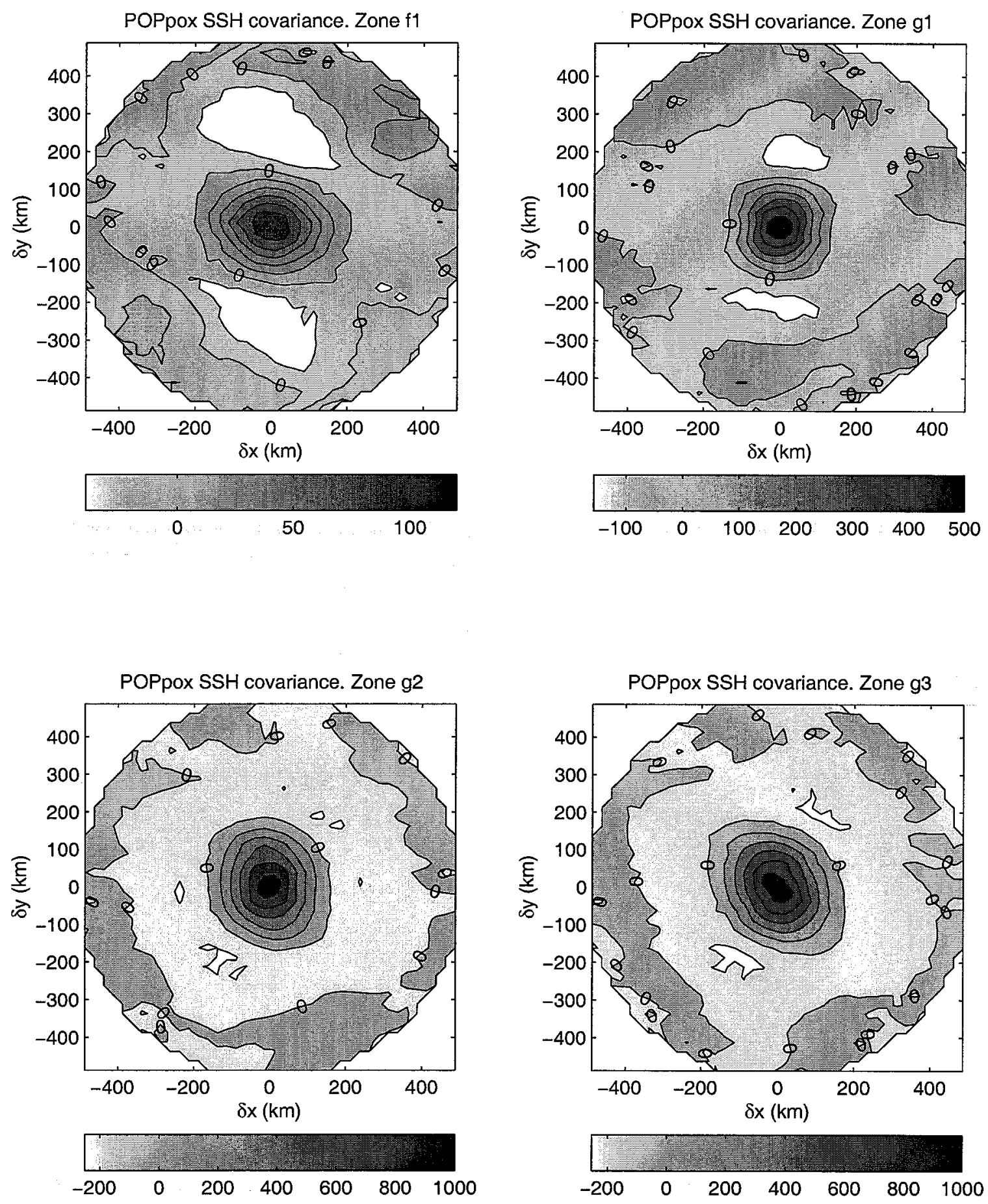

Figure 4-8: Two dimensional covariance for the POP model when sampled along the TOPEX/Poseidon's ground tracks. 

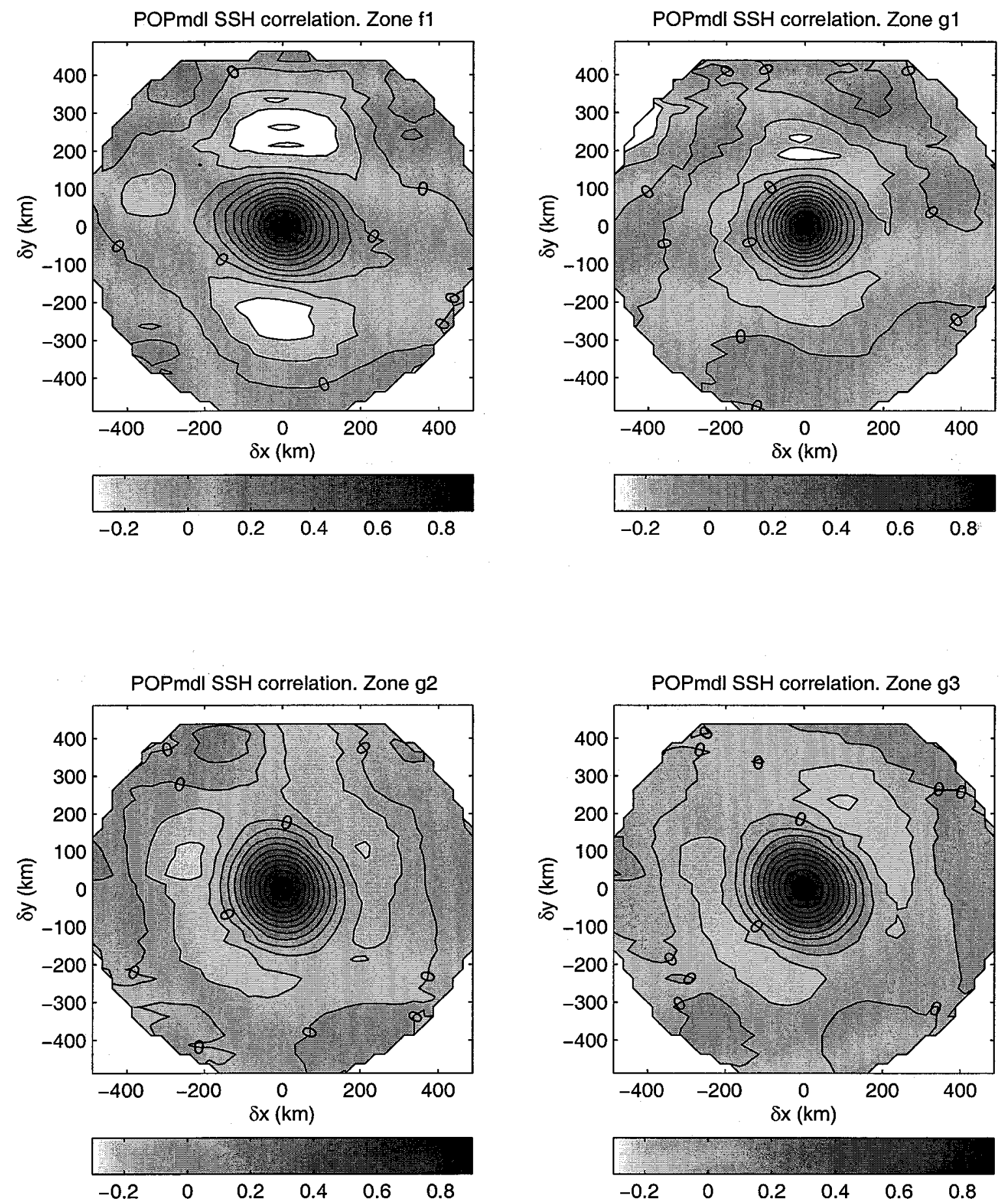

Figure 4-9: Two dimensional correlation using all the numerical model data 

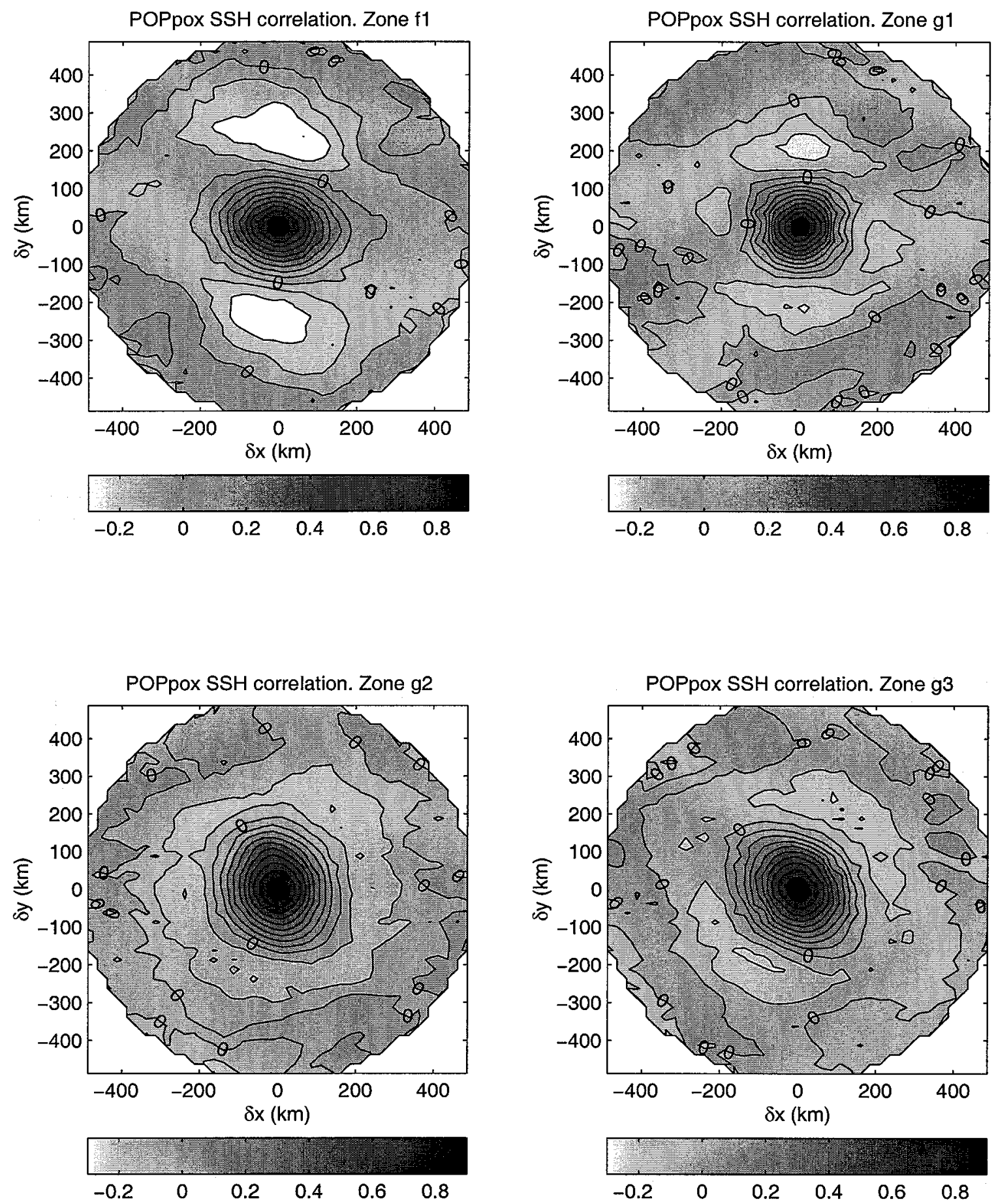

Figure 4-10: Two dimensional correlation for the POP model when sampled along the TOPEX/Poseidon's ground tracks. 
a) confidence interval Zone f1

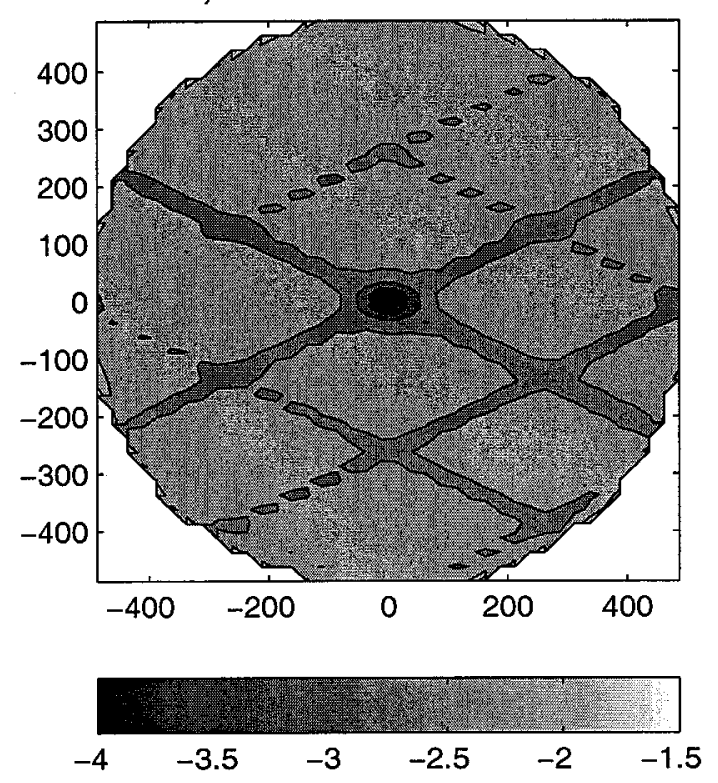

b) confidence interval Zone g2
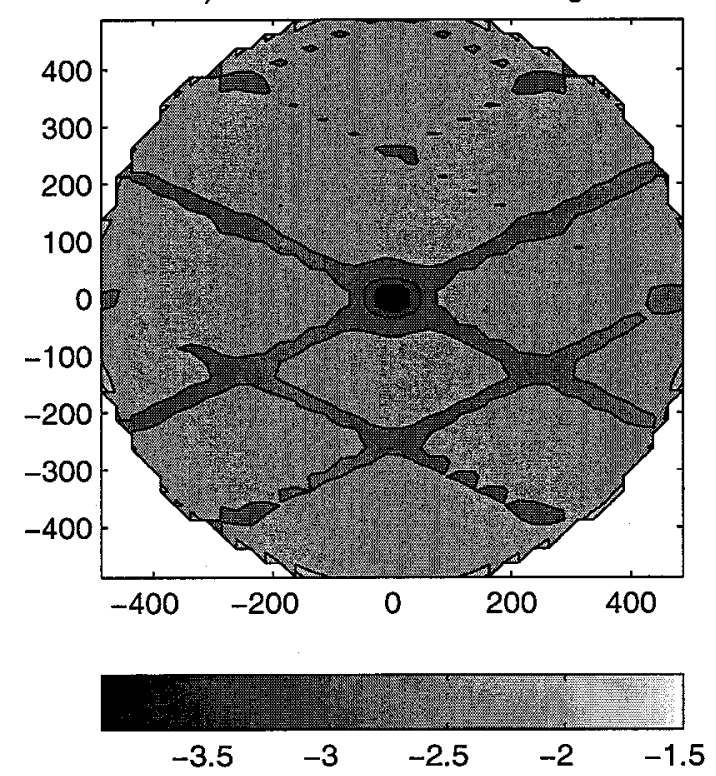

a) confidence interval Zone g1
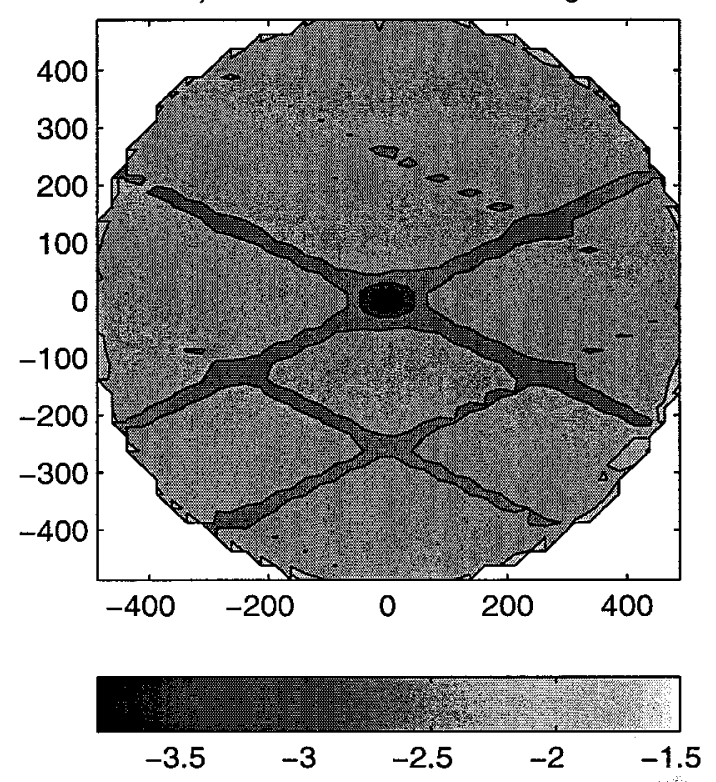

c) confidence interval Zone g3

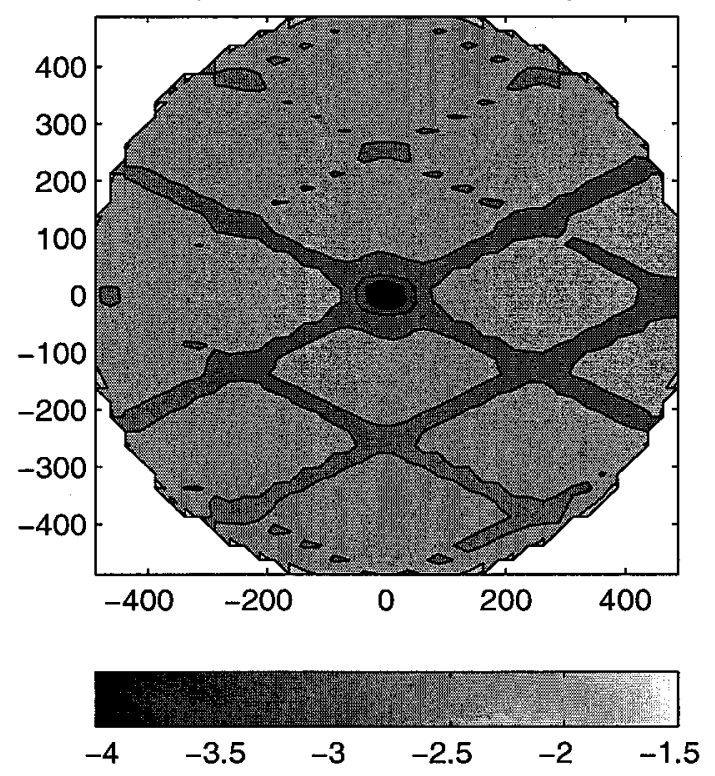

Figure 4-11: Confidence intervals in $\log _{10}$ for the correlations computed with the numerical simulation's sea surface height sampled along the satellite's groundtracks (Figure 4-10) 
Another similarity with the TOPEX results is that the model correlations do not seem to be completely isotropic; the area away from the Gulf stream (f1) appears elongated in the zonal direction. Furthermore, the negative values do not close forming a circle, but instead are limited to the north and south of the center. The symmetry is once again a consequence of the method to compute the correlations, as discussed in section 3.4. The negative values do not seem to be spurious, as they appear in most of the 2-dimensional correlation as well as in most alongtrack ones. The negative values suggest the association of the eddy variability to weaves, and thus the damped periodic characteristics.

The area $\mathrm{f} 1$ could be seen as the extreme of an anisotropic correlation among the ones computed. It has the more negative values to the north and south of the center. The mathematical representation proposed for the correlation (equation 3.16) is still adequate and written again here,

$$
C_{M}=e^{-A_{1} r_{1}^{2}+A_{2} r_{2}^{2}+A_{3} r_{1} r_{2}} \cos \left(\sqrt{B_{1} r_{1}^{2}+B_{2} r_{2}^{2}+B_{3} r_{1} r_{2}}\right)
$$

Figure 4-12 shows a 3-dimensional view of sea surface height correlation for the model f1 area (a), and the mathematical representation (b)when the parameters have been fitted by nonlinear least squares (MATLAB): $A_{1}=1.4 \times 10^{-11}, A_{2}=3.0 \times 10^{-11}, A_{3}=$ $0.7 \times 10^{-11}, B_{1}=11.4 \times 10^{-11}, B_{2}=7.4 \times 10^{-11}, B_{3}=3.4 \times 10^{-11}$. The corresponding contours are shown in (C) and (d), and the residual is shown in (d). The fit is quite good since the residual is small, although they don't seem small when compared to the error bars of the correlation (Figure 4-11 a). The square norm of the residual,

$$
|R|=\sum_{j}^{\hat{N}}\left(C_{M} \mathbf{x}_{j}-C_{D} \mathbf{x}_{j}\right)^{2}
$$

is equal to 3.6 , where $\hat{N}$ is the number of points in the domain 
a) Correlation zone POP-f1

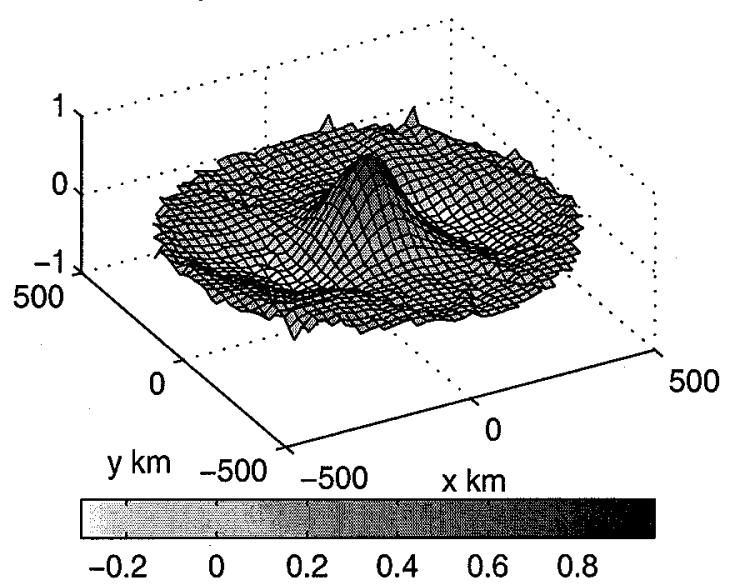

c) contours of( a)

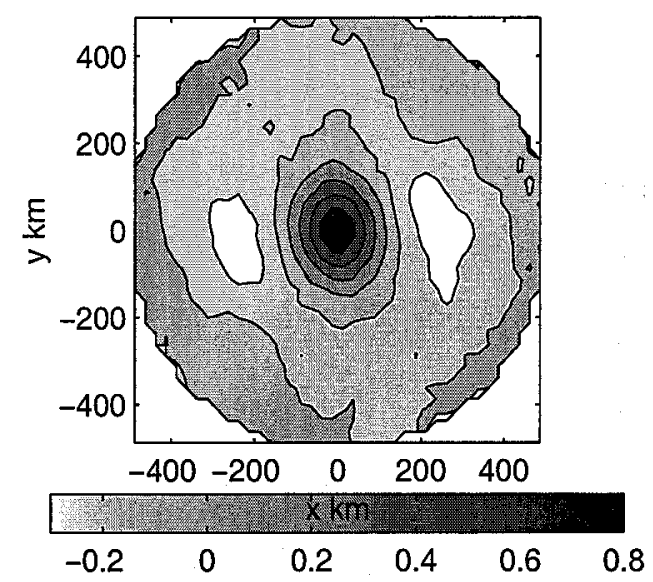

b) Proposed represenation for correlation

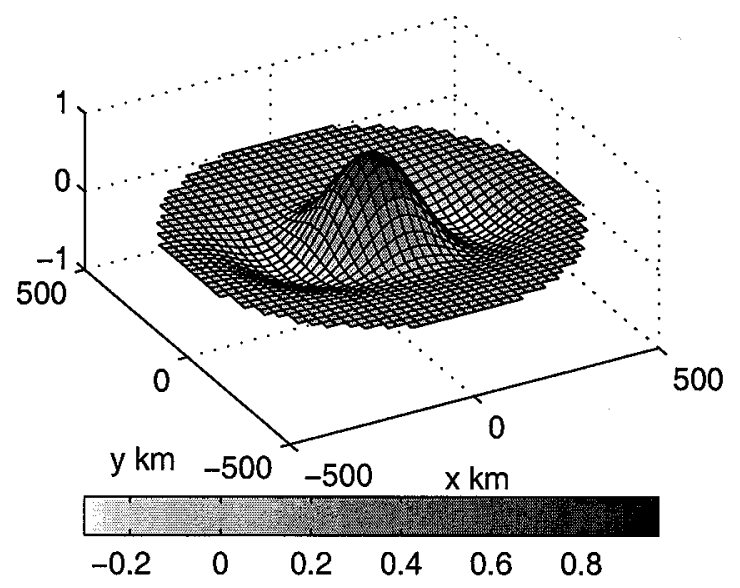

d) contours of (b)

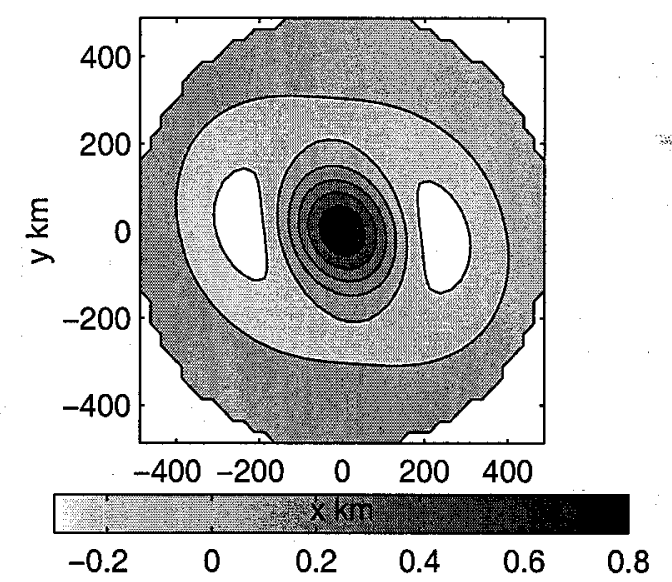

d) residual of the least squares fit

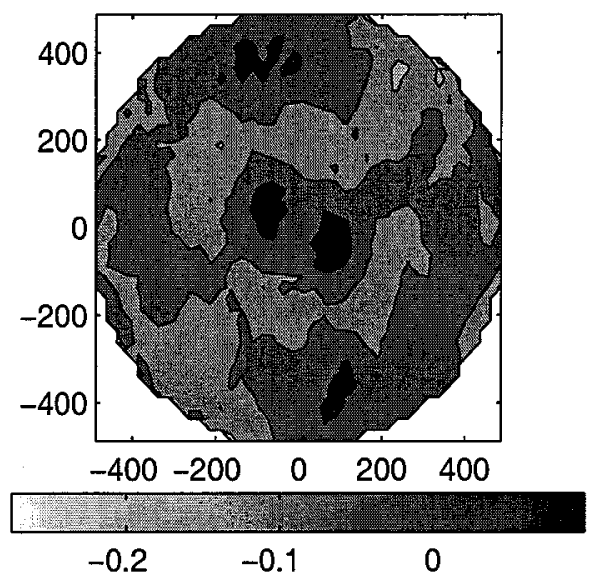

Figure 4-12: 3-dimensional view of the sea surface height correlation for the model f1 area (a), the proposed representation (see text) (b), c) contours of (a), contours of (b), and the residual of the least square fit. 


\subsection{Chapter Summary}

The data processing applied to the TOPEX/Poseidon data is performed on an eddy resolving numerical simulation, and to a subset data formed by the same simulation sampled along the satellite groundtracks. The comparison of both numerical data sets indicate that sampling of TOPEX/Poseidon recovers most of the features analyzed, such as the eddy kinetic energy and sea surface height correlations. A question remains regarding the assumption that the time difference between the measurements from different arcs within a repeat period does not affect much the results.

The eddy kinetic energy computed with the POX data recovers the major features of the real map, given by the full simulation domain, although it does not reproduce the higher values due to the lower resolution provided by the groundtrack crossovers. The The TOPEX/Poseidon eddy kinetic energy has higher values than both model maps, and its high energy area extends further to the east. This comparison between the model data sets suggest that the actual eddy kinetic energy could be larger than inferred from the TOPEX/Poseidon data.

The alongtrack correlations performed are similar in a general sense to the TOPEX/Poseidon results. However, none of the model curves present the slow decay that is found for a few of the curves from the altimeter (Section 3.3, Figure 3-6. I have not found an explanation for such features.

The two-dimensional correlations from the eddy resolving numerical model are similar to the ones computed with TOPEX/Poseidon data. They are close to isotropic, save the bin to the south of the Gulf Stream (f1) of the numerical model that appears to be the more anisotropic. The mathematical representation proposed in section 3.4 proves to be a good approximation of the sea surface height correlation; its parameters are found by a non-linear least squares fit to the numerical simulation correlation function. This expression is used in chapter 5 to compute the eddy vorticity fluxes. 


\section{Chapter 5}

\section{Eddy Field Mean Flow Interaction}

\subsection{Equations of Motion}

In this section, the method for computing the eddy vorticity flux from the stream function spatial covariance function is summarized. A quick review of the equations of motion is first given to clarify the assumptions that are being made; a more formal derivation can be found in Gill (1982), Pedlosky (1987) or similar texts. The method for computing the eddy vorticity flux from the altimetry data will then be detailed.

The equations of motion for a fluid with velocity $u_{i}$, relative to a system that rotates with steady angular velocity $\Omega_{i}$, can be written as,

$$
\frac{\partial u_{i}}{\partial t}+u_{j} \frac{\partial u_{i}}{\partial x_{j}}+2 \epsilon_{i j k} \Omega_{j} u_{k}=-\frac{1}{\rho} \frac{\partial p}{\partial x_{i}}+g_{i}+\nu \nabla^{2} u_{i},
$$

where $\rho$ is the density of the water, $\epsilon_{i j k}$ is the alternating tensor, repeated indices indicate a summation over it (summation convention), and $\nabla^{2}$ is the laplacian operator. A modified pressure, $p$, has been defined such that it includes the effects of pressure and centrifugal forces. The term $2 \epsilon_{i j k} \Omega_{j} u_{k}$ is the Coriolis acceleration that appears due to the system's rotation. It is approximated as $\epsilon_{i 3 k} \Omega_{3} u_{k} \hat{e}_{i}$, where $\hat{e_{i}}$ is a unitary vector in the $i^{\text {th }}$ direction. $\nu$ is the kinematic viscosity, and $g_{i}$ corresponds to the gravity force. I will define $g=g_{3}$ since the gravity acts only in the vertical 
direction.

The relative importance between the inertial, Coriolis, and viscous forces in the horizontal components of the equations can be found by computing the magnitude ratio of the respective terms. Let $U$ be the representative magnitude of the horizontal velocity and $L$ the measure of the horizontal distance over which $U$ varies appreciably; the ratio between the inertial and Coriolis forces defines the Rossby number,

$$
R o=\frac{U}{2 \Omega L}
$$

When $R o \ll 1$ the inertial terms are negligible compared with the Coriolis ones. In a similar way, the ratio of the terms $\nu \nabla^{2} u_{i}$ and $2 \epsilon_{i 3 k} \Omega_{3} u_{k} \hat{e}_{i}$,

$$
E=\frac{\nu}{2 \Omega L^{2}}
$$

known as the Ekman number, measures the relative importance between the viscous and the Coriolis acceleration. The viscous forces in most geophysical fluids, far from the boundary, where viscous forces are important, are negligible with respect to the Coriolis terms $(E \ll 1)$. Equation 5.1 in a steady state with $R o \ll 1$ and $E \ll 1$, states that a balance exists between the Coriolis and pressure terms. This is known as geostrophic balance. When the latitudinal extent is such that the component of the earth's angular velocity on the geographical vertical is constant, $\Omega_{3}=\left|\Omega_{3}\right| \sin (\phi)=$ constant, the Coriolis parameter $f=2 \Omega \sin \left(\phi_{0}\right)$ can be defined, where $\phi_{0}$ is a representative latitude of the region. The expression for the geostrophic balance then takes the form,

$$
\epsilon_{i 3 j} f u_{j}=-\frac{1}{\rho} \frac{\partial p}{\partial x_{i}}
$$

Note that in geostrophic flows the horizontal divergence is equal to zero,

$$
\frac{\partial u_{i}}{\partial x_{i}}=0, \quad i=1: 2,
$$

so a stream function can be defined,

$$
u_{i}=\epsilon_{i 3 j} \frac{\partial \psi}{\partial x_{j}} .
$$


The equation for mass conservation,

$$
\frac{\partial \rho}{\partial t}+u_{i} \frac{\partial \rho}{\partial x_{i}}+\rho \frac{\partial u_{i}}{\partial x_{i}}=0,
$$

in the incompressible case,

$$
\frac{\partial \rho}{\partial t}+u_{i} \frac{\partial \rho}{\partial x_{i}}=0,
$$

states that the vertical velocity is independent of the vertical coordinate, $x_{3}$ since $\frac{\partial u_{3}}{\partial x_{3}}=0$.

When the vertical scale of motion, $l$ is much smaller than the horizontal one, $L \gg l$, the vertical component of the equation of motion establishes what is known as the hydrostatic balance, a balance among the pressure gradient and gravity forces,

$$
\frac{\partial p}{\partial x_{3}}=-\rho g,
$$

\subsubsection{Quasigeostrophic Approximation}

When the fluid is very near geostrophic balance, but not completely, the dynamics of the corrections to the geostrophic balance can be explored. A thorough development of the quasigeostrophic equations is given by Pedlosky (1987); here I will summarize the points relevant for this study.

The Rossby and Ekman numbers are assumed to be much smaller than one. The surface displacement and the bottom slope are considered much smaller than the depth of the fluid at rest. The horizontal scale of motion is extended to the point where the planetary vorticity can no longer be considered constant, but only to the point where it can be modelled as a "small" linear deviation with latitude from a base quantity, $f_{0}=2 \Omega \sin \left(\phi_{0}\right)$, so we have,

$$
f=f_{0}+\beta y,
$$

where $\beta=2 \Omega \cos \left(\phi_{0}\right) / r_{0}$ and $r_{0}$ is the earth's radius. For the deviation with latitude to be small, the latitudinal extension, $y$, is constrained to satisfy $f_{0} \gg \| \beta y \mid$ mainly $L$ 
$l l r_{0}$. For a homogeneous fluid, and after performing an expansion analysis, the geostrophic and hydrostatic balances are recovered at the leading order,

$$
\begin{aligned}
\epsilon_{i 3 j} f u_{j}^{0}=-\frac{1}{\rho} \frac{\partial p^{0}}{\partial x_{i}}, \\
\frac{\partial p^{0}}{\partial x_{3}}=-\rho^{0} g,
\end{aligned}
$$

where the superscript ${ }^{0}$ is used to denote the first order terms. The equations to the next order are,

$$
\frac{\partial u_{i}^{0}}{\partial t}+u_{j}^{0} \frac{\partial u_{i}^{0}}{\partial x_{j}}+\epsilon_{i 3 j} f u_{j}^{1}-\beta u_{i}^{0}=-\frac{1}{\rho} \frac{\partial p^{1}}{\partial x_{i}} .
$$

Where $u_{i}^{1}$ are non-geostrophic horizontal velocity components whose divergence is not zero, as can be seen in the mass conservation equation to second order,

$$
\frac{\partial u_{i}^{1}}{\partial x_{i}}+\frac{\partial u_{3}^{1}}{\partial x_{3}}=0 \quad i=1: 2
$$

The horizontal divergence is compensated by the stretching in the vertical of the water columns.

Since the first order equations are in geostrophic balance, a stream function can be defined as in Equation 5.6. The fluid is considered homogeneous so the stream function can be defined in terms of the sea surface displacement, which allows the use of the satellite data. The hydrostatic balance needs to be integrated in the vertical. The reference frame is such that the z-axis points upwards with the zero at the sea surface under absence of motion. The deviation from this level caused by the motion is called $\eta$. The pressure at the free surface must be zero, $p(\eta)=0$, so after the integration is performed from a level $x_{3}=-z$ we get,

$$
p(-z)=\rho g \eta
$$

When this value is substituted on the geostrophic relation, 5.4 a new set of equations is obtained,

$$
\epsilon_{i k j} f u_{j} \hat{k}=-\frac{1}{\rho} \frac{\partial \eta}{\partial x_{i}}
$$


The stream function can be defined as,

$$
\psi=\frac{g \eta}{f}
$$

\section{Quasigeostrophic vorticity equation}

The quasigeostrophic vorticity equation in steady state is now derived. This will be used to compute the vorticity flux between the turbulent and the mean flows.

The curl of Equation 5.13 is taken, and Equation 5.14 is used to obtain,

$$
\frac{\partial \zeta^{0}}{\partial t}+\frac{\partial\left(u_{i}^{0} \zeta^{0}\right)}{\partial x_{i}}-\beta u_{2}^{0}=f \frac{\partial u_{3}^{1}}{\partial x_{3}}
$$

the quasigeostrophic vorticity equation for an homogeneous fluid in the absence of other forces or dissipation. Here the vertical component of the relative vorticity is defined as $\zeta=\epsilon_{i j 3} \frac{\partial u_{j}}{\partial x_{j}}$. For clarity, the superscript is eliminated from now on, remembering that the horizontal velocities in the equation correspond to the first order that are in geostrophic balance, and that the vertical appears as an ageostrophic correction in the second order.

The flow can be separated in its time mean plus its deviations from the mean, the first denoted by an overbar and the later as a primed quantity. This technique is useful when studying the effects that the turbulent fluctuations might have in the mean flow (Batchelor, 1953) and is sometimes referred as the Reynolds decomposition (Kundu, 1990). The velocity and vertical component of vorticity are,

$$
\begin{aligned}
\mathbf{u}\left(x_{j}, t\right) & =\overline{\mathbf{u}\left(x_{j}\right)_{i}}+\mathbf{u}^{\prime}\left(x_{j} t\right) \\
\zeta\left(x_{j}, t\right) & =\overline{\zeta\left(x_{j}\right)}+\zeta^{\prime}\left(x_{i}, j\right)
\end{aligned}
$$

The summation convention, which states that repeated indices imply a summation, does not apply here. This expression is substituted on the steady state form of Equation 5.18 and the time average is taken. Note that the average of an anomaly is zero by definition. The product of an anomaly times a mean is also zero, however, 
the average of a product of two anomalies can be different from zero. The vorticity equation becomes,

$$
\frac{\partial}{\partial x_{i}}\left(\overline{u_{i} \zeta}\right)+\frac{\partial}{\partial x_{i}}\left(\overline{u_{i}^{\prime} \zeta^{\prime}}\right)-\beta \overline{u_{2}}=f \frac{\partial \overline{u_{3}}}{\partial x_{3}}
$$

The divergence of the eddy relativity flux, the last term on the right, represents an exchange of vorticity between the time dependent and the mean flows. This is the term that can be used to see how the mean flow is influenced by the turbulent flow. The previous expression can be rewritten noting that the horizontal velocity is nondivergent (Equation 5.14) and by dropping the overbar from the mean quantities in Equation 5.19,

$$
u_{i} \frac{\partial}{\partial x_{i}}(f+\zeta)=f \frac{\partial u_{3}}{\partial x_{3}}-\frac{\partial}{\partial x_{i}}\left(\overline{u_{i}^{\prime} \zeta^{\prime}}\right)
$$

The divergence of the eddy vorticity flux, the last term in the right, is now expressed in terms of the velocity covariance. Note that the components of the eddy relative vorticity flux can be expressed as,

$$
\overline{u_{i}^{\prime} \zeta^{\prime}}=\epsilon_{i 3 m} \hat{k} \frac{\partial}{\partial x_{i}} \overline{u_{1}^{\prime} u_{2}^{\prime}}+\frac{1}{2} \frac{\partial}{\partial x_{m}}\left(\overline{u_{2}^{\prime} u_{2}^{\prime}}-\overline{u_{1}^{\prime} u_{1}^{\prime}}\right)
$$

The divergence of the eddy vorticity flux can then be written as,

$$
\frac{\partial}{\partial x_{i}} \overline{u_{i}^{\prime} \zeta^{\prime}}=\frac{\partial^{2}}{\partial x_{1}^{2}} \overline{u_{1}^{\prime} u_{2}^{\prime}}-\frac{\partial^{2}}{\partial x_{2}^{2}} \overline{u_{1}^{\prime} u_{2}^{\prime}}+\frac{\partial^{2}}{\partial x_{1} \partial x_{2}}\left(\overline{u_{2}^{\prime} u_{2}^{\prime}}-\overline{u_{1}^{\prime} u_{1}^{\prime}}\right)
$$

Hogg (1993) proposed a method for the computation of the velocity covariance in terms of the stream function's correlation function in the quasigeostrophic framework. This work is relevant because, under the quasigeostrophic assumption, the stream function can be known from satellite altimetry. Hogg (1993) assumed the correlation function to be horizontally homogeneous and isotropic. His development is written below, were the isotropic constrain in the correlation is relaxed. 


\subsubsection{Eddy vorticity Flux in Terms of the Stream Function Correlation.}

In this section, the velocity covariance will be expressed in terms of the stream function correlation and standard deviation in the quasigeostrophic approximation. Adequate functions with which the correlation and standard deviation can be modelled in the Gulf Stream area will be found and used to compute the eddy vorticity flux divergence of Equation 5.22.

I start by setting two spatially separated points, $x_{i}$ and $\tilde{x_{j}}$, at which the stream function is measured, $\psi\left(x_{i}\right), \tilde{\psi}\left(\tilde{x_{j}}\right)$. In this section, the tildes $(\tilde{x})$ are used to distinguish between the two points. The mean of the stream function has been removed so that only perturbations from it are used. The spatial covariance for the stream function is given by,

$$
F\left(x_{i}, \tilde{x_{j}}\right)=<\psi\left(x_{i}\right), \tilde{\psi}\left(\tilde{x_{j}}\right)>,
$$

where the angled brackets denote an ensemble average. The ensemble averages are computed as time averages (the process is assumed to be ergodic, Bendat and Piersol, 1986); this makes the connection with the covariances in the quasigeostrophic vorticity equation. Note that the covariance is a function of the positions of the two points where the measurements are taken.

As before, the flow is horizontally non-divergent since it is in geostrophic balance at leading order, so a stream function can be defined as in Equation 5.6. The velocity covariance can then be written in terms of the stream function,

$$
<u_{i}, \tilde{u_{j}}>=\epsilon_{i 3 m} \epsilon_{j 3 n} \frac{\partial^{2} F}{\partial x_{m} \partial \tilde{x_{n}}} .
$$

The correlation coefficient $f\left(x_{i}\right)$ is defined as the normalized covariance by the product of the standard deviations at each point (historically, the terms covariance and correlation have had different uses and the literature sometimes can be confusing, as Batchelor (1953) noted. Bendat \& Piersol (1986) define, for example, the correlation function as the covariance of a stationary process). Hogg (1993) assumed that 
the correlation coefficient of the stream function was homogeneous and isotropic, in which case it is a function of the separation distance between the points where the measurements are obtained, $\left|r_{i}\right|=\sqrt{\left(\tilde{x}_{i}-x_{i}\right)^{2}}$. It will be assumed here that the correlation coefficient is homogeneous but that it can be anisotropic. In this case the correlation is a function of the relative position among the measurement points, $r_{i}=\tilde{x}_{i}-x_{i}$. With this, the correlation function can be written as,

$$
f\left(r_{i}\right)=\frac{F\left(x_{i}, \tilde{x_{i}}\right)}{S\left(x_{i}\right) S\left(\tilde{x_{i}}\right)},
$$

where the standard deviation is given by,

$$
S\left(x_{i}\right)=\sqrt{<\psi\left(x_{i}\right)^{2}>} .
$$

The TOPEX/Poseidon data suggest that the assumption of a homogeneous correlation is a good approximation (see Section 3.4; the area along the Gulf stream has much larger variability than the area away from it, so the covariance is also larger, but it seems homogeneous after the normalization by the standard deviation product. The data suggests also that the correlation is approximately isotropic, but not completely (Section 3.4. The effect that the anisotropy has in the vorticity flux will be explored.

When the covariance expression from 5.25 is substituted on the velocity covariance, 5.24 , it results in,

$$
<u_{i}, \tilde{u_{j}}>=\epsilon_{i 3 m} \epsilon_{j 3 n}\left[\frac{\partial S}{\partial x_{m}} \frac{\partial \tilde{S}}{\partial \tilde{x_{n}}} f\left(r_{i}\right)+\frac{\partial S}{\partial x_{m}} \tilde{S} \frac{\partial f\left(r_{i}\right)}{\partial \tilde{x_{n}}}+S \frac{\partial \tilde{S}}{\partial \tilde{x_{n}}} \frac{f\left(r_{i}\right)}{\partial x_{m}}+S \tilde{S} \frac{\partial^{2} f\left(r_{i}\right)}{\partial x_{m} \partial \tilde{x_{n}}}\right] .
$$

The distance between the two points where the stream function is measured can be made as small as possible to find the velocity covariance at one point. The limit when $\mathbf{r} \rightarrow 0$ will be applied, but first we will transform the derivatives of the correlation on $x$ and $\tilde{x}$ in derivates on $r$. Note that since $r_{i}=\tilde{x_{i}}-x_{i}$,

$$
\begin{gathered}
\frac{\partial f}{\partial \tilde{x_{m}}}=\frac{\partial f}{\partial r_{m}} \frac{\partial r_{m}}{\partial \tilde{x}_{i}}=\frac{\partial f}{\partial r_{m}}, \\
\frac{\partial f}{\partial x_{i}}=\frac{\partial f}{\partial r_{i}} \frac{\partial r_{i}}{\partial x_{i}}=-\frac{\partial f}{\partial r_{i}},
\end{gathered}
$$


then the covariance at one point takes the form,

$$
\lim _{\mathbf{r} \rightarrow 0}\left\langle u_{i}, u_{j}\right\rangle=\epsilon_{i 3 m} \epsilon_{j 3 n}\left[\frac{\partial S}{\partial x_{m}} \frac{\partial S}{\partial r_{n}} f(0)+\frac{\partial S}{\partial x_{m}} S \frac{\partial f(0)}{\partial r_{n}}-S \frac{\partial S}{\partial x_{n}} \frac{f(0)}{\partial r_{m}}-S S \frac{\partial^{2} f(0)}{\partial r_{m} \partial r_{n}}\right] .
$$

Now that the velocity covariance has been expressed in terms of the stream function correlation and standard deviation in the quasigeostrophic approximation, An adequate mathematical representation of the correlation and standard deviation for the Gulf Stream area is sought, so that the eddy vorticity flux divergence can be computed. It will also be needed to find the expression of their derivatives in order to substitute them in Equation 5.28.

The altimetry data suggests that the correlation function can be modelled by the product of a gaussian function and a sinusoid, (Section 3.4, where Equation 3.16 is rewritten here)

$$
f\left(r_{i}\right)=e^{-A_{1} r_{1}^{2}+A_{2} r_{2}^{2}+A_{3} r_{1} r_{2}} \cos \left(\sqrt{B_{1} r_{1}^{2}+B_{2} r_{2}^{2}+B_{3} r_{1} r_{2}}\right) .
$$

The first derivative of the correlation on one component of $\mathbf{r}$ is

$$
\frac{\partial f}{\partial r_{m}}=-\left\{\cos (\sqrt{\beta})\left(2 A_{m} r_{m}+A_{3} r_{\hat{m}}\right)+\frac{\sin (\sqrt{\beta})}{\sqrt{\beta}}\left(B_{m} r_{m}+\frac{1}{2} B_{3} r_{\hat{m}}\right)\right\} e^{-\alpha},
$$

where $\hat{m}=3-m$, and

$$
\begin{gathered}
\alpha=A_{1} r_{1}^{2}+A_{2} r_{2}^{2}+A_{3} r_{1} r_{2}, \\
\beta=B_{1} r_{1}^{2}+B_{2} r_{2}^{2}+B_{3} r_{1} r_{2}
\end{gathered}
$$

have been defined for the clarity in the notation.

The second derivative of the correlation with respect to, either the same component of $\mathbf{r}$ or the other one, is

$$
\begin{aligned}
\frac{\partial^{2} f}{\partial r_{m} \partial r_{n}}= & -\left\{\left(2 A_{m} r_{m}+A_{3} r_{\hat{m}}\right) \frac{\partial}{\partial x_{n}} \cos (\sqrt{\beta})+\cos (\sqrt{\beta}) \frac{\partial}{\partial x_{n}}\left(2 A_{m} r_{m}+A_{3} r_{\hat{m}}\right)\right\} e^{-\alpha} \\
& +\left\{\left(B_{m} r_{m}+\frac{1}{2} B_{3} r_{\hat{m}}\right) \frac{\partial}{\partial x_{n}} \frac{\sin (\sqrt{\beta})}{\sqrt{\beta}}+\frac{\sin (\sqrt{\beta})}{\sqrt{\beta}} \frac{\partial}{\partial x_{n}}\left(B_{m} r_{m}+\frac{1}{2} B_{3} r_{\hat{m}}\right)\right\} e^{-\alpha} \\
& +\frac{\partial \alpha}{\partial x_{n}}\left\{\cos (\sqrt{\beta})\left(2 A_{m} r_{m}+A_{3} r_{\hat{m}}\right)+\frac{\sin (\sqrt{\beta})}{\sqrt{\beta}}\left(B_{m} r_{m}+\frac{1}{2} B_{3} r_{\hat{m}}\right)\right\} e^{-\alpha},
\end{aligned}
$$


where $\hat{n}=3-n$. We now write explicitly the derivatives and regroup into similar terms,

$$
\begin{aligned}
\frac{\partial^{2} f}{\partial r_{m} \partial r_{n}}= & \left\{\left(\frac{\sin (\sqrt{\beta})}{\sqrt{\beta}}\left(B_{m} r_{m}+\frac{1}{2} B_{3} r_{\hat{m}}\right)+\cos (\sqrt{\beta})\left(2 A_{m} r_{m}+A_{3} r_{\hat{m}}\right)\right)\left(2 A_{n} r_{n}+A_{3} r_{\hat{n}}\right)\right\} e^{-\alpha} \\
& -\left\{\frac{\sin (\sqrt{\beta})}{\sqrt{\beta}}\left(B_{m} \delta_{m n}+\frac{1}{2} B_{3}\left(\delta_{m n}-1\right)\right)+\cos (\sqrt{\beta})\left(2 A_{m} \delta_{m n}+A_{3}\left(\delta_{m n}-1\right)\right)\right\} e^{-\alpha} \\
& -\left\{\left(\cos (\sqrt{\beta})-\frac{\sin (\sqrt{\beta})}{\sqrt{\beta}}\right)\left(\frac{B_{n} r_{n}+\frac{1}{2} B_{3} r_{\hat{n}}}{\beta}\right)\left(B_{m} r_{m}+\frac{1}{2} B_{3} r_{\hat{m}}\right)\right\} e^{-\alpha} \\
& +\left\{\frac{\sin (\sqrt{\beta})}{\sqrt{\beta}}\left(2 A_{m} r_{m}+A_{3} r_{\hat{m}}\right)\left(B_{n} r_{n}+\frac{1}{2} B_{3} r_{\hat{n}}\right)\right\} e^{-\alpha}
\end{aligned}
$$

We apply the limit when $\mathbf{r} \rightarrow 0$ to the Equations $5.29,5.29$, and 5.30 so they can be substituted on Equation 5.28. Note that

$$
\begin{aligned}
\lim _{\mathbf{r} \rightarrow 0} \frac{\sin (\sqrt{\beta})}{\sqrt{\beta}} & =\lim _{\mathbf{r} \rightarrow 0} \operatorname{sinc}(\sqrt{\beta})=1 \\
\lim _{\mathbf{r} \rightarrow 0} \cos (\sqrt{\beta}) & =\lim _{\mathbf{r} \rightarrow 0} e^{-\alpha}=1 \\
\lim _{\mathbf{r} \rightarrow 0} \frac{r_{i} r_{j}}{\beta} & =0
\end{aligned}
$$

so we get

$$
\begin{aligned}
\lim _{\mathbf{r} \rightarrow 0} f\left(r_{i}\right) & =1 \\
\lim _{\mathbf{r} \rightarrow 0} \frac{\partial f}{\partial r_{m}} & =0 \\
\lim _{\mathbf{r} \rightarrow 0} \frac{\partial^{2} f}{\partial r_{m} \partial r_{n}} & =-\left(2 A_{m}+B_{m}\right) \delta_{m n}-\left(A_{3}+\frac{1}{2} B_{3}\right)\left(\delta_{m n}-1\right)
\end{aligned}
$$

With the aid of the first two expressions in 5.31, the velocity covariance taken at one point, Equation 5.28, can be written as,

$$
<u_{i}, u_{j}>=\epsilon_{i 3 m} \epsilon_{j 3 n}\left[\frac{\partial S}{\partial x_{m}} \frac{\partial S}{\partial x_{n}}-S^{2} \frac{\partial^{2} f(0)}{\partial x_{m} \partial x_{n}}\right] .
$$

This expression can be used to get the divergence of the eddy vorticity flux by substituting in Equation 5.22, which I rewrite here for convenience:

$$
\frac{\partial}{\partial x_{i}} \overline{u_{i}^{\prime} \zeta^{\prime}}=\frac{\partial^{2}}{\partial x_{1}^{2}} \overline{u_{1}^{\prime}, u_{2}^{\prime}}-\frac{\partial^{2}}{\partial x_{2}^{2}} \overline{u_{1}^{\prime} u_{2}^{\prime}}+\frac{\partial^{2}}{\partial x_{1} \partial x_{2}}\left(\overline{u_{2}^{\prime} u_{2}^{\prime}}-\overline{u_{1}^{\prime}, u_{1}^{\prime}}\right)
$$


I will be explicit with the algebra; each term is expanded.

a) The first two terms on the right side,

$$
\begin{gathered}
\frac{\partial^{2}}{\partial x_{1}^{2}}<u_{1}^{\prime}, u_{2}^{\prime}>-\frac{\partial^{2}}{\partial x_{2}^{2}}<u_{1}^{\prime}, u_{2}^{\prime}>=\underbrace{-\underbrace{\frac{\partial S}{\partial x_{2}} \frac{\partial^{3} S}{\partial x_{1}^{3}}}}_{1} \\
\underbrace{-2 \frac{\partial^{2} S}{\partial x_{1}^{2}} \frac{\partial^{2} S}{\partial x_{1} \partial x_{2}}}_{2} \underbrace{-\frac{\partial S}{\partial x_{1}} \frac{\partial^{3} S}{\partial x_{1}^{2} \partial x_{2}} \underbrace{+\frac{\partial S}{\partial x_{2}} \frac{\partial^{3} S}{\partial x_{1} \partial x_{2}^{2}}}_{4}}_{3} \\
\underbrace{+2 \frac{\partial^{2} S}{\partial x_{1} \partial x_{2}} \frac{\partial^{2} S}{\partial x_{2}^{2}}}_{5} \underbrace{+\frac{\partial S}{\partial x_{1}} \frac{\partial^{3} S}{\partial x_{2}^{3}}}_{6}+\underbrace{\left(\frac{\partial^{2}\left(S^{2}\right)}{\partial x_{1}^{2}}-\frac{\partial^{2}\left(S^{2}\right)}{\left.\partial x_{2}^{2}\right)}\right) \frac{\partial^{2} f(0)}{\partial x_{1} \partial x_{2}}}_{7}
\end{gathered}
$$

b) The last term on the right side becomes,

$$
\begin{aligned}
& \frac{\partial^{2}}{\partial x_{1} \partial x_{2}}\left(<u_{2}^{\prime}, u_{2}^{\prime}>-<u_{1}^{\prime}, u_{1}^{\prime}>\right)=\underbrace{2 \frac{\partial^{2} S}{\partial x_{1}^{2}} \frac{\partial^{2} S}{\partial x_{1} \partial x_{2}} \underbrace{+2 \frac{\partial S}{\partial x_{1}} \frac{\partial^{3} S}{\partial x_{1}^{2} \partial x_{2}}}_{3}}_{2} \\
& \underbrace{-2 \frac{\partial^{2} S}{\partial x_{1} \partial x_{2}} \frac{\partial^{2} S}{\partial x_{2}^{2}}}_{5} \underbrace{-2 \frac{\partial S}{\partial x_{2}} \frac{\partial^{3} S}{\partial x_{1} \partial x_{2}^{2}}}_{4}+\underbrace{2\left(\frac{\partial^{2} f(0)}{\partial x_{2}^{2}}-\frac{\partial^{2} f(0)}{\partial x_{1}^{2}}\right)\left(\frac{\partial S}{\partial x_{1}} \frac{\partial S}{\partial x_{2}}+S \frac{\partial^{2} S}{\partial x_{1} \partial x_{2}}\right)}_{8}
\end{aligned}
$$

The expression for the eddy vorticity flux is found by adding the two previous equalities,

$$
\begin{aligned}
\frac{\partial}{\partial x_{i}}<u_{i}^{\prime} \zeta^{\prime}>= & \frac{\partial S}{\partial x_{1}} \nabla^{2}\left(\frac{\partial S}{\partial x_{2}}\right)-\frac{\partial S}{\partial x_{2}} \nabla^{2}\left(\frac{\partial S}{\partial x_{1}}\right)+\frac{\partial^{2}\left(S^{2}\right)}{\partial x_{1} \partial x_{2}}\left(\frac{\partial^{2} f(0)}{\partial x_{2}^{2}}-\frac{\partial^{2} f(0)}{\partial x_{1}^{2}}\right) \\
& +\left(\frac{\partial^{2}\left(S^{2}\right)}{\partial x_{1}^{2}}-\frac{\partial^{2}\left(S^{2}\right)}{\partial x_{2}^{2}}\right) \frac{\partial^{2} f(0)}{\partial x_{1} \partial x_{2}} .
\end{aligned}
$$

The expression for the derivatives of $f$ can be substituted from Equation 5.31,

$$
\begin{aligned}
\frac{\partial}{\partial x_{i}}<u_{i}^{\prime} \zeta^{\prime}>= & \frac{\partial S}{\partial x_{1}} \nabla^{2}\left(\frac{\partial S}{\partial x_{2}}\right)-\frac{\partial S}{\partial x_{2}} \nabla^{2}\left(\frac{\partial S}{\partial x_{1}}\right)+\frac{\partial^{2}\left(S^{2}\right)}{\partial x_{1} \partial x_{2}}\left[2\left(A_{1}-A_{2}\right)+B_{1}-B_{2}\right] \\
& -\left(A_{3}+\frac{1}{2} B_{3}\right)\left(\frac{\partial^{2}\left(S^{2}\right)}{\partial x_{1}^{2}}-\frac{\partial^{2}\left(S^{2}\right)}{\partial x_{2}^{2}}\right) .
\end{aligned}
$$

The last terms vanish for the isotropic case since $A_{1} \equiv A_{2}, B_{1} \equiv B_{2}$, and $A_{3}=B_{3} 0$,

For the more general case, the anisotropy contributes through,

$$
\frac{\partial^{2}\left(S^{2}\right)}{\partial x_{1} \partial x_{2}}\left[2\left(A_{1}-A_{2}\right)+B_{1}-B_{2}\right]-\left(A_{3}+\frac{1}{2} B_{3}\right)\left(\frac{\partial^{2}\left(S^{2}\right)}{\partial x_{1}^{2}}-\frac{\partial^{2}\left(S^{2}\right)}{\partial x_{2}^{2}}\right) .
$$


These terms might cancel for particular values of $A_{i}$ and $B_{i}$, but in general there is a contribution to the eddy vorticity flux by the anisotropy.

To explore what consequences the anisotropy might produce in the vorticity balance, the anisotropic case will be compared with the isotropic case developed by Hogg (1993).

\subsection{Vorticity Balance for the Gulf Stream Region}

Here, the quasigeostrophic time averaged vorticity equation (Equation 5.18) is developed for the Gulf Stream geometry. The eddy vorticity flux in terms of the stream function's statistics, (Equation 5.34), when the correlation is modelled as the product of a gaussian function times a cosine (Equation 5.29), is used to compute the vorticity balance. The majority of this was done by Hogg (1993).

Observations suggest that recirculation gyres near the Gulf Stream are barotropic (Schmitz, 1980, Hogg et al., 1986). If they are assumed to be so, but superimposed on a baroclinic zonal jet, the time averaged vorticity Equation 5.18 can be integrated in the vertical from the bottom $x_{3}=-D\left(x_{i}\right)$ to the surface, $x_{3}=0$,

$$
U_{i} \int_{-D}^{0} \frac{\partial \bar{\zeta}}{x_{i}} d x_{3}+U_{2} \beta D=f\left(u_{i}(-D) \frac{\partial D}{\partial x_{i}}\right)-\int_{-D}^{0} \frac{\partial \overline{u_{i}^{\prime} \zeta^{\prime}}}{\partial x_{i}}
$$

where barotropic velocity is defined,

$$
U_{i}=\frac{1}{D} \int_{-D}^{0} u_{i} d x_{3} .
$$

Hogg (1993) simplifies further the expression by examining the current meter data from the SYNOP (Synoptic Ocean Prediction Experiment) mooring array located between $36^{\circ}-42^{\circ} \mathrm{N}$, and east of $55^{\circ} \mathrm{W}$ from 1987 to 1989 . The relative vorticity of the time mean flow is smaller than the planetary and topography induced vorticities. It is also found that the meridional gradient of the relative vorticity is always positive, and that the topography slopes upwards towards the north enhancing $\beta$. He defines 
an effective $\beta, \beta^{*}=4 \times 10^{-11} s^{-1} m^{-1}$ that sums all this contributions to the vorticity balance. At the end he gets a modified Sverdrup balance for the mean flow,

$$
\beta^{*} V=-\frac{1}{D} \int_{-D}^{0} \frac{\partial}{\partial x_{i}} \overline{u_{i}^{\prime} \zeta^{\prime}} d z
$$

where for clarity $V=U_{2}, x_{3}=z$. The depth-averaged meridional flow is forced by the divergence of the eddy relative vorticity flux, which can be estimated by the correlation and standard deviation of the stream function as we have seen. With the aid of Equation 5.34, and expressing the zonal velocity in term of the stream function, the modified Sverdrup balance is written as

$$
\begin{aligned}
\beta^{*} & \frac{\partial \psi}{\partial x_{1}}=-\frac{1}{D} \int_{-D}^{0} \nabla\left\{\frac{\partial S}{\partial x_{1}} \nabla^{2}\left(\frac{\partial S}{\partial x_{2}}\right)-\frac{\partial S}{\partial x_{2}} \nabla^{2}\left(\frac{\partial S}{\partial x_{1}}\right)\right\} d z \\
& -\frac{1}{D} \int_{-D}^{0}\left\{\frac{\partial^{2}\left(S^{2}\right)}{\partial x_{1} \partial x_{2}}\left[2\left(A_{1}-A_{2}\right)+B_{1}-B_{2}\right]\right\} d z \\
+ & \frac{1}{D} \int_{-D}^{0}\left\{\left(A_{3}+\frac{1}{2} B_{3}\right)\left(\frac{\partial^{2}\left(S^{2}\right)}{\partial x_{1}^{2}}-\frac{\partial^{2}\left(S^{2}\right)}{\partial x_{2}^{2}}\right)\right\} d z .
\end{aligned}
$$

From the stream function standard deviation distribution, Figure 5-1, it can be seen that its meridional scale is much smaller than the zonal one. The following approximations can be made,

$$
\begin{aligned}
\nabla^{2} S & \approx \frac{\partial^{2} S}{\partial x_{2}^{2}} . \\
\frac{\partial^{2}\left(S^{2}\right)}{\partial x_{1}^{2}}-\frac{\partial^{2}\left(S^{2}\right)}{\partial x_{2}^{2}} & \approx-\frac{\partial^{2}\left(S^{2}\right)}{\partial x_{2}^{2}}
\end{aligned}
$$

It will also be assumed that the horizontal scales of the correlation are of the same magnitude as the meridional scale of the standard deviation. Equation 5.39 becomes,

$$
\begin{aligned}
\beta^{*} \frac{\partial \psi}{\partial x_{1}}= & -\frac{1}{D} \int_{-D}^{0}\left(\frac{\partial S}{\partial x_{1}} \frac{\partial^{3} S}{\partial x_{2}^{3}}-\frac{\partial S}{\partial x_{2}} \frac{\partial^{3} S}{\partial x_{1} \partial x_{2}^{2}}+\frac{\partial^{2}\left(S^{2}\right)}{\partial x_{1} \partial x_{2}}\left[2\left(A_{1}-A_{2}\right)+B_{1}-B_{2}\right]\right) d z \\
& -\frac{1}{D} \int_{-D}^{0}\left(A_{3}+\frac{1}{2} B_{3}\right) \frac{\partial^{2}\left(S^{2}\right)}{\partial x_{2}^{2}} d z
\end{aligned}
$$

An integration in the meridional direction is needed to evaluate the stream function. A simple analytical expression was obtained for the isotropic case by Hogg 
(1993). The same development can be done for the case in which $A_{m}, B_{m} \neq 0$, for $m=1: 2$, and $A_{3}=B_{3}=0$. This case will be done for for clarity, and a numerical integration will be done for the more general case in which $A_{3}$ and $B_{3} \neq 0$.

\subsubsection{Anisotropy Oriented Zonally or Meridionally}

For the case in which the anisotropy is present only in the zonal or meridional direction $\left(A_{i}, B_{i} \neq 0, i=1: 2\right.$, but $\left.A_{3}=B_{3}=0\right)$ an integration in the zonal direction can be performed on Equation 5.42 assuming the stream function's standard derivation can be separated, following Hogg (1993), into

$$
s\left(x_{1}, x_{2}\right)=G\left(x_{1}\right)\left(x_{2}\right)
$$

If this expression is substituted into 5.42 ,

$\beta^{*} \frac{\partial \psi}{\partial x_{1}}=-\frac{1}{2 D} \int_{-D}^{0}\left(\frac{\partial G^{2}}{\partial x_{1}}\left[H \frac{\partial^{3} H}{\partial x_{2}^{3}}-\frac{\partial H}{\partial x_{2}} \frac{\partial^{2} H}{\partial x_{2}^{2}}+2 \frac{\partial H^{2}}{\partial x_{2}}\left[2\left(A_{1}-A_{2}\right)+B_{1}-B_{2}\right]\right]\right) d z$

which can be integrated in the zonal direction to obtain an expression for the component of the time averaged stream function produced by the eddy vorticity flux,

$$
\psi=-\frac{1}{2 D \beta^{*}} \int_{-D}^{0}\left(S \frac{\partial^{3} S}{\partial x_{2}^{3}}-\frac{\partial S}{\partial x_{2}} \frac{\partial^{2} S}{\partial x_{2}^{2}}+2 \frac{\partial S^{2}}{\partial x_{2}}\left[2\left(A_{1}-A_{2}\right)+B_{1}-B_{2}\right]\right) d z
$$

The altimetry data suggests that the stream function's standard deviation can be approximated by a gaussian function,

$$
S=A e^{-\left(c_{1} x_{1}^{2}+c_{2} x_{2}^{2}\right)}
$$

Figure 5-1 shows the contours for the stream function's standard deviation computed from the altimetric data (a) and the analytical given by expression 5.46 with the

parameters, $A=45000 \mathrm{~m}^{-2} \mathrm{~s}^{-1}, c_{1}=4 x 10^{-13} \mathrm{~m}^{-2}$, and $c_{2}=1.8 \times 10^{11} \mathrm{~m}^{-2}$. It seems that the analytical standard deviation reproduces the general characteristics of the one derived from the satellite's data. There is a small positive tilt in the altimetric 
contours which are not found in the analytical ones. The simple expression will be kept for the moment, a further analysis needs to be done to see the effects of the small tilt.

Note that $C_{1} \ll C_{2}$, an approximation that made in expression 5.40. Equation 5.45 becomes, after substituting the stream function's standard deviation formula,

$$
\psi=-\frac{4 C_{2}}{\beta^{*}} x_{2} S^{2}\left(C_{2}+2\left(A_{1}-A_{2}\right)+B_{1}-B_{2}\right) .
$$

Hoggs (1993) result is recovered for the isotropic case, in which $A_{1}=A_{2}, B_{1}=B_{2}$. Figure 5-2 (a) shows the transport associated with the streamfunction when a $4 \mathrm{~km}$ depth is used and the parameters are, $A_{1}=A_{2}, B_{1}=B_{2}, A_{3}=B_{3}=0$. The actual values of the parameters $A_{i}, i=1: 2$ do not matter since the mean stream function forced by the eddy vorticity flux depends only on the stream function standard deviation. The eddy field drives two recirculation gyres, a cyclonic to the north and anticyclonic to the south, with a maximum transport of near $20 S v$, consistent with Hogg's (1993) results.

The effect of the anisotropy in the zonal or meridional direction can be analyzed using the parameters of the stream function correlation found in sections 3.4 for the satellite data and Section 4.3 for the numerical simulation. The anisotropic term is different from the isotropic by the coefficients $C_{2}$, for the first one, and $2\left(A_{1}-A_{2}\right)+B_{1}-B_{2}$ for the latter (Equation 5.47). Figure 5-2(b) show the component of the anisotropy in the zonal and meridional direction with the parameters for the area g2 of the TOPEX/Poseidon analysis obtained in section 3.4: $A_{1}=2.9 \times 10^{-11} \mathrm{~m}^{-2}, A_{2}=3 \times 10^{-11} \mathrm{~m}^{-2}, B_{1}=12 \times 10^{-11} \mathrm{~m}^{-2}, B_{2}=10 \times 10^{-11} \mathrm{~m}^{-2}$, and $A_{3}=B_{3}=0$. The anisotropy in the meridional and zonal direction does not seem to be large since $A_{1} / A_{2}=0.94 \neq 1$ and $B_{1} / B_{2}=1.2$; however, its contribution is very similar to that of the isotropic component, $C_{2}=1.8 \times 10^{-11} \mathrm{~m}^{-2}$ and $2\left(A_{1}-A_{2}\right)+B_{1}-B_{2}=1.5 \times 10-11 m^{-2}$.

The value $2\left(A_{1}-A_{2}\right)+B_{1}-B_{2}$ can cancel for anisotropic cases in which $A_{1} \neq$ $A_{2}, B_{1} \neq B_{2}$, but $2\left(A_{1}-A_{2}\right)=B_{2}-B_{1}$. The area $\mathrm{f} 1$ of the numerical model is 
a) TOPEX stream function standard deviation. $\mathrm{m}^{2} \mathrm{~s}^{-1}$

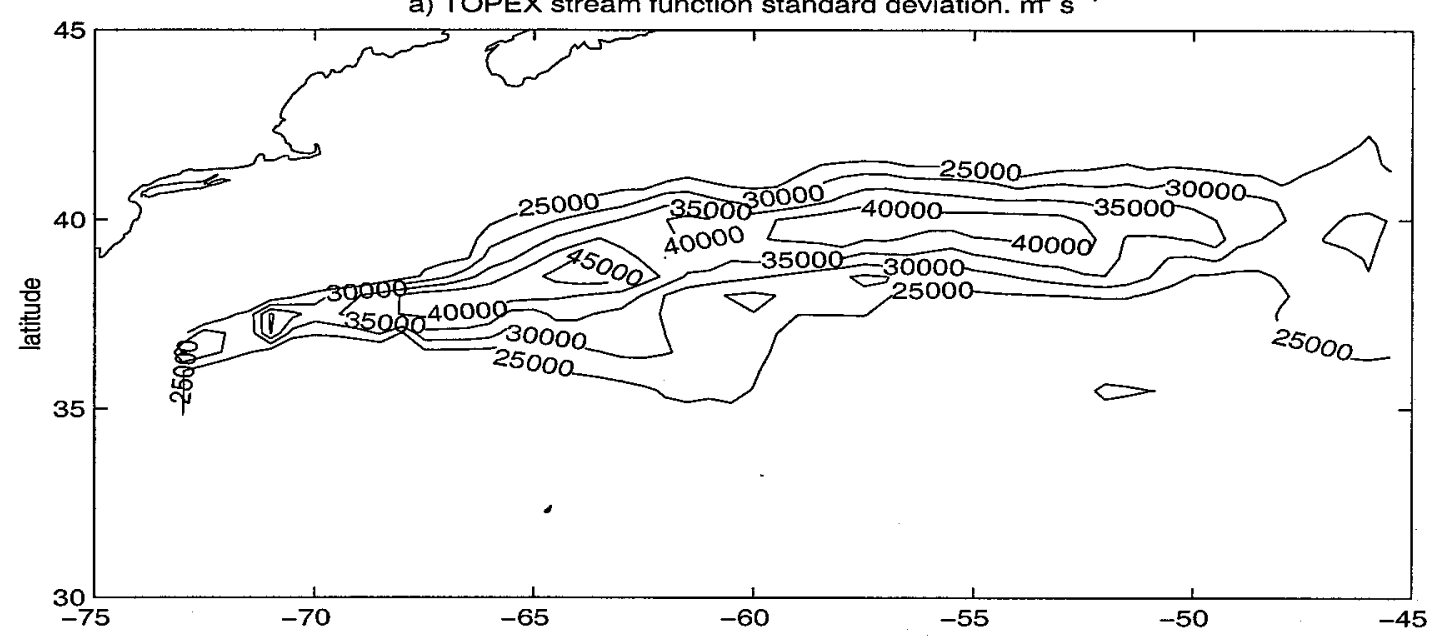

b) Analytic stream function

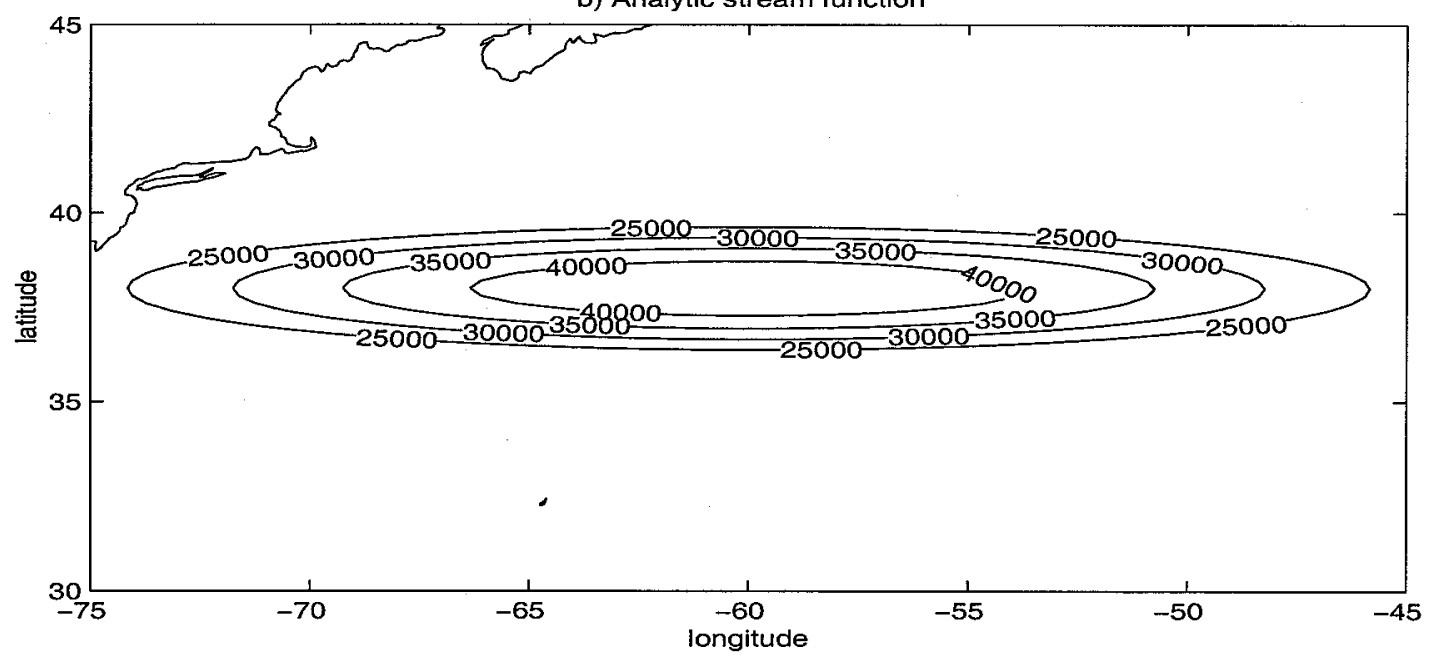

Figure 5-1: Stream function's standard deviation from a) TOPEX/Poseidon data and b) the analytic form. Contours above $25000 \mathrm{~cm}^{2} \mathrm{~s}^{-1}$ 


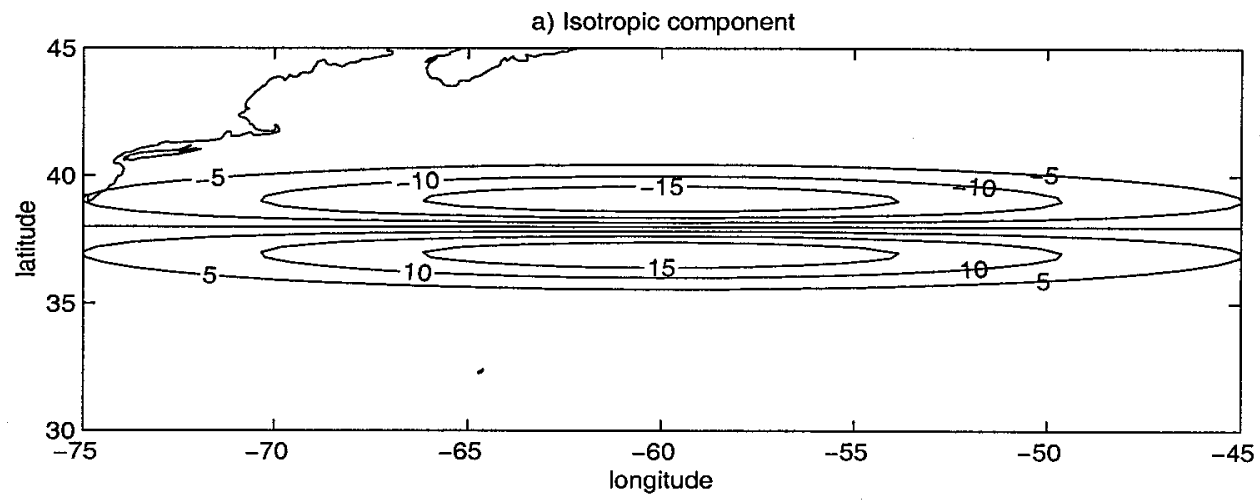

b) Zonal-meridional anisotropic component

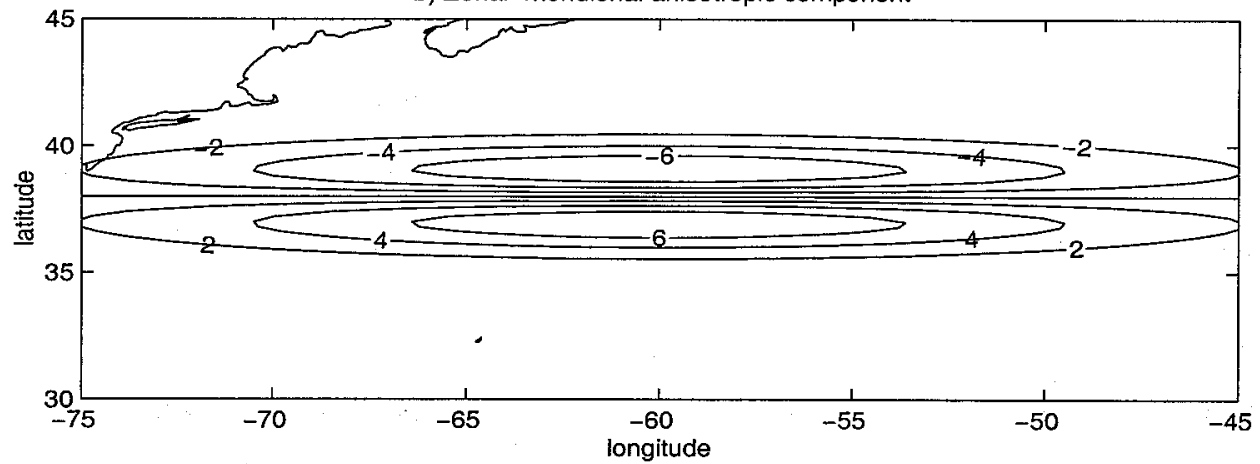

c) Diagonal anisotropy component

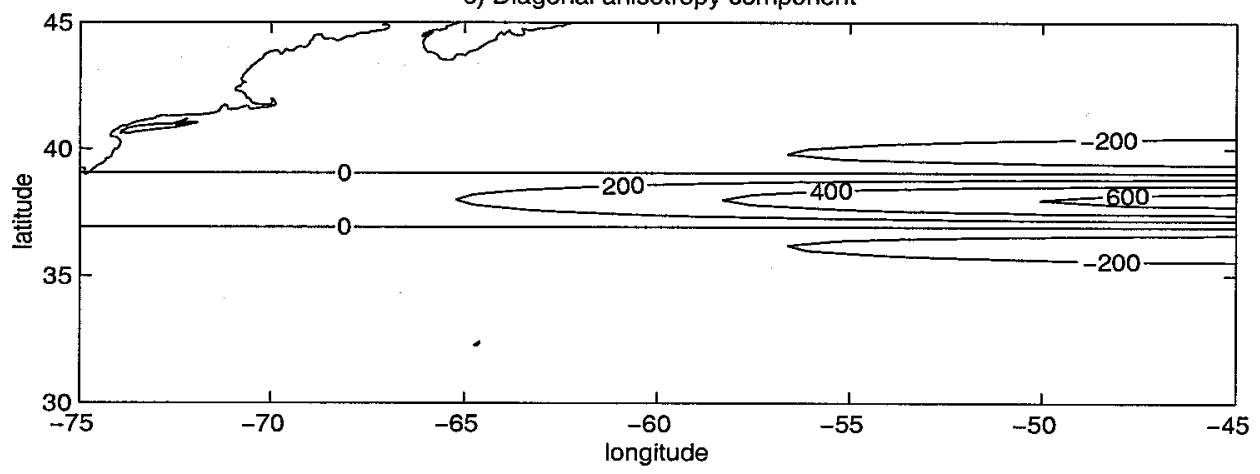

Figure 5-2: transport in sverdrups $\left(1 x 10^{6} \mathrm{~m}^{3} \mathrm{~s}^{-1}\right)$ forced by the eddy vorticity flux when the stream function correlation of the bin g2 of the TOPEX/Poseidon is used. The isotropic component (a), component for anisotropy in meridional or zonal direction (b), and the diagonal anisotropy component (c). 
more anisotropic than TOPEX/Poseidon g2, but its contribution is smaller. The parameters for the area $\mathrm{f} 1$ of the numerical simulation are $A_{1}=1.4 \times 10^{-11} \mathrm{~m}^{-2}, A_{2}=$ $3 \times 10^{-11} m^{-2}, A_{3}=-0.7 \times 10^{-11} m^{-2}, B_{1}=11 \times 10^{-11} m^{-2}, B_{2}=7 \times 10^{-11} m^{-2}$, and $B_{3}=$ $3.4 \times 10^{-11} m^{-2}$ (see Section 4.3), but $A_{3}=B_{3}=0$ are considered in this section. The parameters are not that different from the area g2 of TOPEX/Poseidon, but they indicate that the correlation is more anisotropic, as can be seen by the ratios $A_{1} / A_{2}=0.46$ and $B_{1} / B_{2}=1.53$. Even though the $\mathrm{f1}$ area is more anisotropic, its value $2\left(A_{1}-A_{2}\right)+B_{1}-B_{2}=7.3 \times 10^{-11}$ is half the value than for TOPEX/Poseidon area g2. Figure 5-3 shows the isotropic and anisotropic components of the transport induced by the eddy vorticity flux. Not surprisingly, the anisotropic component is smaller than the satellite $\mathrm{g} 2$ isotropic component despite of being more anisotropic.

\subsubsection{Diagonal Anisotropy.}

The diagonal anisotropy is included by letting $A_{3}, B_{3} \neq 0$. In this case a numerical integration is needed since it cannot be done analytically.

The least squares fit for the TOPEX/Poseidon g2 area results in $A_{3}=-0.2 \times 10^{-11} m^{-2}, B_{3}=$ $3.3 \times 10^{-11} \mathrm{~km}^{-2}$. Figure 5-2 (c) shows the result of the integration of the anisotropy in the diagonal term, which yields streamlines that do not close and transports in excess of $300 S v$. This has not been observed near the Gulf Stream and it points out to an error in the assumptios made, that the correlations are homogeneous. A similar result is obtained for the $\mathrm{f1}$ area of the numerical model, which has $A_{3}=$ $-0.7 \times 10^{-11} \mathrm{~m}^{-2}, B_{3}=3.4 \times 10^{-11} \mathrm{~km}^{-2}$. Figure $5-3$ (c) shows again open streamlines with maximum transport greater than $600 S v$.

The expression that is integrated in the meridional direction is an even function, which accounts for the open streamlines. From Equation 5.42 and expression 5.46,

$$
\frac{\partial^{2}\left(S^{2}\right)}{\partial x_{2}^{2}}\left(A_{3}+\frac{1}{2} B_{3}\right)=16 C_{2}^{2} x_{2}^{2} S^{2}\left(A_{3}+\frac{1}{2} B_{3}\right)+4 C_{2} S^{2}\left(A_{3}+\frac{1}{2} B_{3}\right)
$$




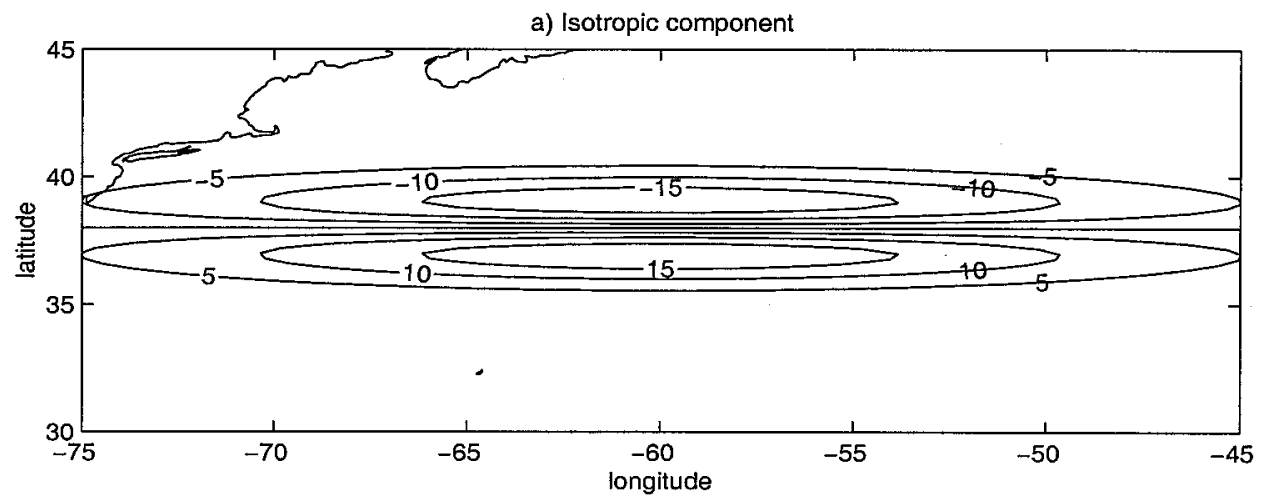

b) Zonal-meridional anisotropic component

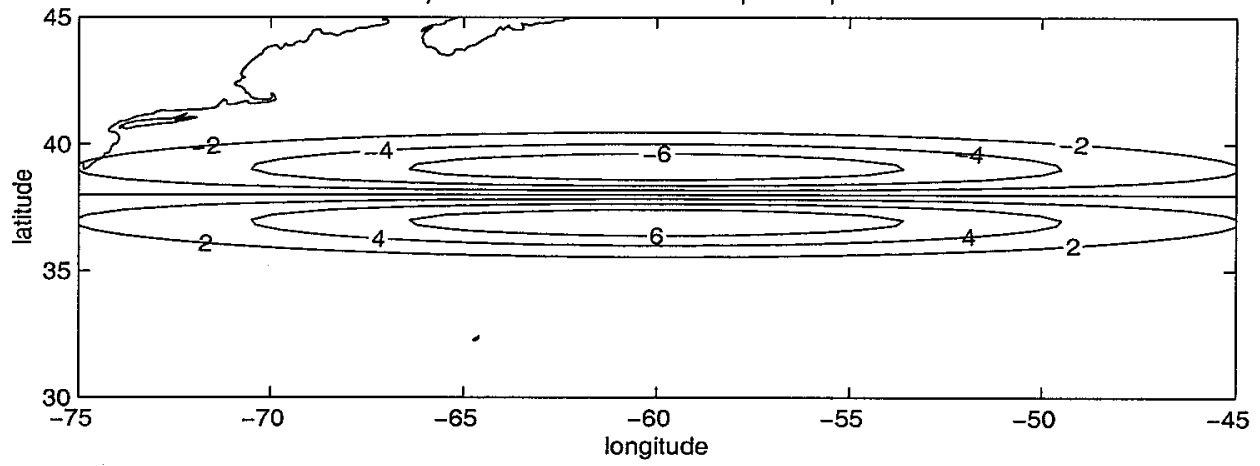

c) Diagonal anisotropy component

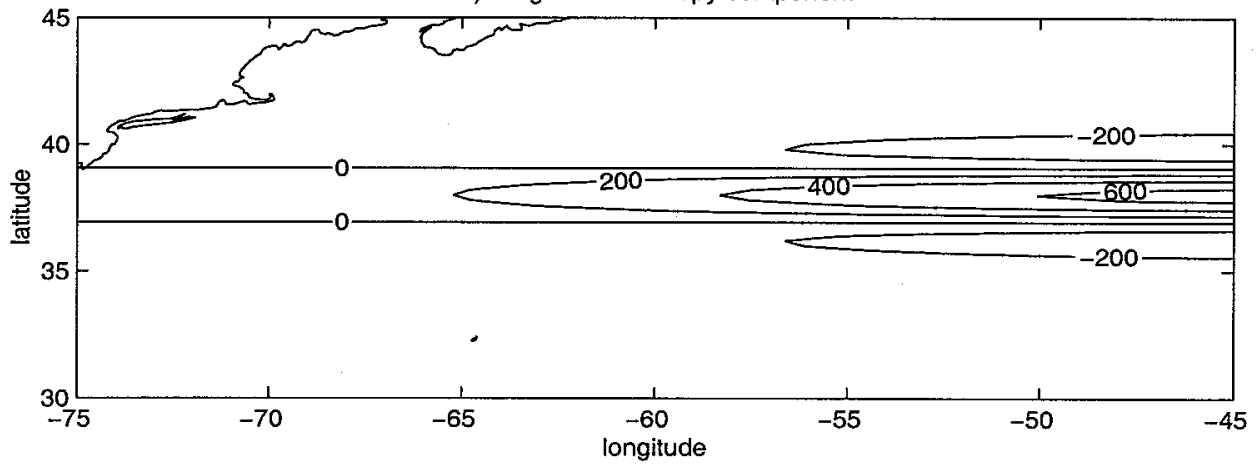

Figure 5-3: transport in sverdrups $\left(1 \times 10^{6} \mathrm{~m}^{3} \mathrm{~s}^{-1}\right)$ forced by the eddy vorticity flux when the stream function correlation of the bin $\mathrm{fl}$ of the numerical simulation is used. The isotropic component (a), component for anisotropy in meridional or zonal direction (b), and the diagonal anisotropy component (c). 
while the expression for the isotropic case, which is odd, produces closed contours,

$$
\frac{\partial S}{\partial x_{1}} \frac{\partial^{3} S}{\partial x_{2}^{3}}-\frac{\partial S}{\partial x_{2}} \frac{\partial^{3} S}{\partial x_{1} \partial x_{2}^{2}}=16 C_{2}^{2} x_{2} S^{2} C_{1} x_{1} .
$$

It has been assumed that the correlation function is homogeneous. It could be that this assumption is not satisfied and that small inhomogeneities prevent the use of one stream function correlation for the whole domain. What is evident, is that small anisotropy can change largely the associated transport.

\subsection{Chapter Summary}

The stream function is defined in terms of the sea surface height when the flow is considered horizontally non-divergent and homogeneous. The eddy vorticity flux can then be expressed in terms of the stream function standard deviation and correlation function.

The standard deviation is approximated by a gaussian function. The correlation function of the stream function is approximated mathematically by the product of a cosine and a gaussian function in chapters 3 and 4 . The parameters of the mathematical expression are found by fitting to the correlation function by a nonlinear least squares formulation (MATLAB).

The eddy vorticity flux depends entirely on the stream function standard deviation when the stream function correlation is isotropic. The eddy kinetic flux found from the inferred standard deviation drives two recirculation gyres, cyclonic to the north of the Gulf Stream axis and anticyclonic to the south, with a maximum transport close to 20Sv.

The eddy vorticity flux changes by the same order of magnitude when the correlation function of the stream function is anisotropic in the zonal or meridional direction. In this case, the eddy vorticity flux is a function of the stream function standard deviation and correlation function. It is found that small anisotropies have a big impact 
on the circulation. A modest anisotropic example produced two recirculation gyres very similar to the isotropic case.

The method for computing the eddy vorticity flux breaks down when the anisotropy in the diagonal direction, assuming it is homogeneous, is included. Open contours of the stream function with associated transports in the order of $600 S v$ are found. This suggest that the homogeneous assumption needs to be revised, and that the anisotropy, even when small, has a large impact on the eddy vorticity fluxes. 


\section{Chapter 6}

\section{Discussion and Conclusion}

Comparison between the eddy kinetic anergy and sea surface height correlation from an eddy resolving numerical simulation and from the same data set subsampled along TOPEX/Poseidon groundtracks, suggest that the satellite resolves acceptably the general features of the eddy kinetic energy and the sea surface height correlation. The assumption of velocity isotropy is not needed for the computation of the eddy kinetic energy from the sea surface height. The velocity components are found at the satellite groundtrack crossovers. The resolution thus obtained does not resolve completely all the features of the eddy kinetic energy field and can underestimate it magnitude, nevertheless, the general shape and magnitude are well resolved.

The eddy field mean flow interaction is studied near the Gulf Stream by analyzing the eddy vorticity flux in the quasigeostrophic framework by means of satellite altimetry and an eddy resolving numerical simulation. The eddy vorticity flux is expressed in terms of the stream function standard deviation and correlation function (Hogg,1993). The stream function is defined in terms of the sea surface height measured by the satellite, or form the numerical simulation, assuming the flow is homogeneous, in near geostrophic balance, and hydrostatic.

The streamfunction's standard deviation is modelled as a gaussian function based 
in the map constructed with the satellite data

$$
S=e^{-\left(C_{1} x_{1}^{2}+C_{2} x_{2}^{2}\right)}
$$

The altimetric stream function correlation is similar to the model stream function correlation, and does not follow a gaussian distribution. Negative values are present at $100-300 \mathrm{~km}$ from the origin. The stream function correlation is modelled herein as

$$
C_{M}=e^{-A_{1} r_{1}^{2}+A_{2} r_{2}^{2}+A_{3} r_{1} r_{2}} \cos \left(\sqrt{B_{1} r_{1}^{2}+B_{2} r_{2}^{2}+B_{3} r_{1} r_{2}}\right)
$$

The parameters are found by fitting the function to the stream function correlation of the data by nonlinear least squares. The correlations are roughly homogeneous and close to isotropic. The computation of the eddy vorticity flux show that the small deviations from the homogeneous and isotropic case are important.

Two recirculation gyres are found near the Gulf Stream Extension for the isotropic case, consistent with Hogg (1993). The gyres, a cyclonic to the north of the current's axis and cyclonic to the south, have a maximum transport of around 20Sv. The eddy potential vorticity flux depends entirely on the stream function standard deviation and its spatial derivatives.

The anisotropy in the meridional or zonal direction can modify the circulation induced by the eddy vorticity flux. In one analyzed case, the anisotropy was found to drive two recirculation gyres similar in strength and position to the isotropic case. The eddy potential vorticity flux depends in this case of the stream function standard deviation, the streamfunction correlation function and their spatial derivatives, although, the correlation can be anisotropic in the meridional or zonal direction and have no contribution to the eddy vorticity flux. A necessary condition for the anisotropy to have any effect is $2\left(A_{1}-A_{2}\right)+B_{1}-B_{2} \neq 0$. This anisotropic contribution is different from the isotropic case by the coefficient $2\left(A_{1}-A_{2}\right)+B_{1}-B_{2}$ instead of $C_{1}$. Small departures from isotropy that have $2\left(A_{1}-A_{2}\right)+B_{1}-B_{2}$ of the same order of magnitude as $C_{1}$, have a large impact on the eddy vorticity flux. 
Anisotropy in the diagonal direction is not successfully modelled. Stream line contours that do not close on themselves and high transports $(>600 S v)$ are found when the diagonal anisotropy is included. The stream function correlation is assumed homogeneous. The small inhomogeneity, neglected in this study, could prove to be important and be responsible for the error in the eddy vorticity flux calculation when diagonal anisotropy is included. 


\section{Appendix A}

\section{Orbit Error.}

A detailed explanation of the orbit error follows because it was thought at one point to be of particular importance in the present work.

The orbit error is defined as the error in the radial component of the orbit ephemerides (Cutting et al. 1978). For the first altimetric missions, this error was about $1.5 \mathrm{~m}$ (Seasat), and was produced by the inaccuracy of the gravity models and problems with the tracking systems (Marsh and Williamson, 1980). Mayor improvements have been achieved for TOPEX/Poseidon, reducing the orbit error to $2 \mathrm{~cm}$ ( $\mathrm{Lu}$ \& Cazenave, 2001) and rendering the gravity model and tracking system to a less important role (Tapley et al. 1994). Some authors consider that the orbit error has been reduced to the level where corrections are no longer required (Lu and Cazenave, 2001) while others consider that despite the improvements, even the orbit error as small as in TOPEX/Poseidon can adversely affect certain altimeter applications (Marshall et al. 1995); in the present work, for example, along-track correlations that are never significantly different from zero have been found, which were thought to be explained by the orbit error (Tai, personal communication, 2000).

There have been many different attempts to remove the orbit error from the altimetric data. Most of the orbit error power manifests itself as a time dependent long-scale function with predominant spectral peak at 1 cycle-per-revolution $(1 \mathrm{cpr}$, 
equivalent to the earth's circumference, 40000km) (Marsh and Williamson, 1980). With this in mind, the first attempts to remove the orbit error consisted on adjusting a large scale function, which represented the orbit error, to along-track data segments of length much smaller than the earth's circumference. Cheney and Marsh (1983) adjusted a linear trend to $2000 \mathrm{~km}$ length arcs to study mesoscale variability near the Gulf Stream using the collinear data of the Seasat mission.

In a method developed later, called the crossover adjustment, a long-scale function is also fitted to each along-track data. In this case, the parameters of the function are calculated by minimizing the difference in sea surface height measured by two different transects at the same point. The transects used in the calculation of the crossover difference can be of the same revolution or correspond to different revolutions (Tai, 1988). The functions that have been used are polynomials of up to second order for regional adjustments, i.e., transects much smaller than 40,000km (Gordon et al. 1983; Tai, 1989), a sinusoidal function with 40,000km wavelength (Tai, 1988) and Fourier series truncated to selected frequency (Tai and Fu, 1986; Tai and Kuhn, 1994).

The polynomial fit with cross-over adjustment not only eliminates the 1cpr peak, but also affects the oceanographic signals (Tai, 1991; Tai and Wagner, 1994). A better choice for the global adjustment is the the Fourier series as it preserves better the oceanographic signals and can eliminate more of the errors (Tai and Kuhn, 1994, 1995). For a regional adjustment the polynomial or sinusoidal fit work well (Tai, personal communication, 2000). We will find below the practical difference between them and which one is more convenient.

\section{Sinusoidal Crossover Adjustment}

The sinusoidal crossover adjustment is set up as a least squares problem. The orbit error for each transect is represented as the sum of a sine, cosine and a constant. The difference in sea surface height due to the orbit error for each crossover is

$$
d_{i j}=\left[a_{j} \cos \left(2 \pi t_{j} / T\right)+b_{j} \sin \left(2 \pi t_{j} / T\right)+c_{j}\right]-\left[a_{i} \cos \left(2 \pi t_{i} / T\right)+b_{i} \sin \left(2 \pi t_{i} / T\right)+c_{i}\right]
$$


where $i, j$ stand for the transect. This equations are to be solved by least squares; they can be written in matrix notation as,

$$
\mathbf{E x}+\mathbf{n}=\mathbf{y}
$$

where the matrix $\mathbf{E}$ contains the values of the sinusoidal functions evaluated at the corresponding time (position), the vector $\mathbf{x}$ corresponds to the unknown values of the coefficients $a$ 's, $b$ 's, and c's, the vector $\mathbf{y}$ stands for the crossover differences, and $\mathbf{n}$, usually called noise, to the crossover differences not due to the orbit error. The approach consists on minimizing the noise,

$$
J=\left(\mathbf{n}^{\mathbf{T}} \mathbf{n}\right)=(\mathbf{y}-\mathbf{E x})^{T}(\mathbf{y}-\mathbf{E x})
$$

even though the noise can contain oceanographic signals and other errors or mismatches due to time difference between the transects. The system called normal equations is obtained by differentiating (A.3) with respect to $\mathrm{x}$ and equating to zero,

$$
\mathbf{E}^{\mathbf{T}} \mathbf{E x}=\mathrm{E}^{\mathbf{T}} \mathbf{y}
$$

whose solution, assuming the inverse exists, is

$$
\mathbf{x}=\left(\mathbf{E}^{\mathbf{T}} \mathbf{E}\right)^{-1} \mathbf{E}^{\mathbf{T}} \mathbf{y}
$$

Superscript $\left({ }^{T}\right)$ denotes the transpose of the matrix and $\left({ }^{-1}\right)$ its inverse.

The solution to Equation A.2 is not unique since there might be components of the orbit error that do not manifest themselves as sea surface height difference at the cross over. This components can not be resolved by solving for (A.2). In other words, there might be a subspace of $\mathbf{x}$, called the nullspace of $\mathbf{E}$, that satisfy $\mathbf{E x} \mathbf{x}^{\prime}=0$. The matrix $\mathbf{E}$ is then said to be singular and the inverse of $\left(\mathbf{E}^{\mathbf{T}} \mathbf{E}\right)$ can not be found directly.

The non uniqueness of the solution can be removed by minimizing the solution (Tai, 1988, Tai and Kuhn, 1994). Tapered least squares and singular value decomposition (SVD) are two almost equivalent methods that provide a mean to control the 
norm of the solution against that of the noise, and can therefore be used to minimize the solution and eliminate the ill-condition character of $\mathbf{E}$ (Lawson and Hanson, 1974; Wunsch, 1996). A combination of the methods was used in this work. A description of each method follows, and hte combined method is described at the end. Many texts describe both methods. I chose to follow Lawson and Hanson (1974) and Wunsch (1996).

\section{Singular Value Decomposition.}

Singular value decomposition (SVD) gives the solution to (A.2) as its expansion in an orthonormal spanning set. It uses singular value theory to find the spanning vectors of the the range of $\mathbf{E}$, and the expansion coefficients for $\mathbf{x}$. A brief review is given that will help understand how can it help solve (A.2) when the matrix is singular or ill conditioned.

A square symmetric matrix can be formed from any $M \mathrm{x} N$ matrix as follows:

$$
\mathbf{B}=\left[\begin{array}{cc}
0 & E^{T} \\
E & 0
\end{array}\right] \text {. }
$$

The singular value problem for bf $\mathrm{B}$ can be written as $\mathbf{B} \mathbf{q}_{i}=\lambda_{i} \mathbf{q}_{i}$. Since the matrix $\mathbf{B}$ has many zeros, it is convenient to define:

$$
\mathbf{v}_{i}=\left[\begin{array}{c}
\mathbf{q}_{i} \\
: \\
\mathbf{q}_{N i}
\end{array}\right], \quad \mathbf{u}_{i}=\left[\begin{array}{c}
\mathbf{q}_{N+1, i} \\
: \\
\mathbf{q}_{M i}
\end{array}\right], \quad \mathbf{q}_{i}=\left[\begin{array}{c}
\mathbf{v}_{i} \\
\mathbf{u}_{i}
\end{array}\right] \text {, }
$$

so $\mathbf{B q}_{i}=\lambda_{i} \mathbf{q}_{i}$ can be separated into

$$
\begin{aligned}
\mathbf{E} \mathbf{v}_{i} & =\lambda_{i} \mathbf{u}_{i} \\
\mathbf{E}^{T} \mathbf{u}_{i} & =\lambda_{i} \mathbf{v}_{i}
\end{aligned}
$$

This two expressions can be combined to get two independent singular problems:

$$
\begin{aligned}
& \mathbf{E E}^{T} \mathbf{u}_{i}=\lambda_{i}^{2} \mathbf{v}_{i} \\
& \mathbf{E}^{T} \mathbf{E} \mathbf{v}_{i}=\lambda_{i}^{2} \mathbf{u}_{i} .
\end{aligned}
$$


for which $\mathbf{u}_{i}$ and $\mathbf{v}_{i}$ are singular vectors; each of them forms a complete orthonormal spanning set for their respective spaces ( $\mathbf{u}_{i}$ for an $\mathrm{M}$ dimension space and $\mathbf{v}_{i}$ of $\mathrm{N}$ dimension). They can be used to expand $\mathbf{x}, \mathbf{y}$, and $\mathbf{n}$ :

$$
\mathbf{x}=\sum_{i=1}^{N} \alpha_{i} \mathbf{v}_{i}, \quad \mathbf{y}=\sum_{i=1}^{M} \beta_{i} \mathbf{u}_{i}, \quad \mathbf{n}=\sum_{i=1}^{M} \gamma_{i} \mathbf{u}_{i}
$$

The value of the expansion coefficient for $\mathbf{x}$ need to be found to get the solution to (A.2). The value of $\mathbf{y}$ is known, since it is measured, and can there for be written, $\beta_{i}=\mathbf{u}_{i}^{T} \mathbf{y}$. Substituting on the original least square equation, (A.2),

$$
\sum_{i=1}^{N} \alpha_{i} \operatorname{Ev}_{i}+\sum_{i=1}^{M} \gamma_{i} \mathbf{u}_{i}=\sum_{i=1}^{M} \mathbf{u}_{i}^{T} \mathbf{y u}
$$

In the general case, $M \neq N$, so $\mathbf{u}_{i}$ and $\mathbf{v}_{i}$ have different dimensions; some $\lambda_{i}$ need to be equal to zero for (A.8) to be consistent. In fact, $\lambda_{i} \neq 0, i>\min (M, N)$. Let $k$ be the indice up to which the singular values are different from zero. Substituting (A.8) on (A.13) with $\lambda_{i} \neq 0, i \leq k$,

$$
\sum_{i=1}^{k} \alpha_{i} \mathbf{E v}_{i}+\sum_{i=1}^{M} \gamma_{i} \mathbf{u}_{i}=\sum_{i=1}^{M} \mathbf{u}_{i}^{T} \mathbf{y} \mathbf{u}^{T} .
$$

Note that the summations have different upper limit. One of the advantages of this method is that it produces a solution even when the matrix is singular. By the orthonormality of the singular vectors we have,

$$
\alpha_{i} \lambda_{i}+\gamma_{i}=\mathbf{u}_{i}^{T} \mathbf{y}
$$

We can solve for $\alpha_{i}$ where $\lambda_{i} \neq 0,1 \leq i \leq k$ by setting $\mathbf{u}_{i}^{T} \mathbf{y}=0$. This corresponds to minimize the noise norm, the same requirement for least squares,

$$
\alpha_{i}=\frac{\mathbf{u}_{i}^{T} \mathbf{y}}{\lambda_{i}}, \quad 1 \leq i \leq k .
$$

The only solution for $\lambda_{i}=0, i>k$ is $\gamma_{i}=\mathbf{u}_{i}^{T} \mathbf{y}$ and the corresponding $\alpha_{i}$ are undetermined. The solution found is 


$$
\begin{array}{r}
\hat{\mathbf{x}}=\sum_{i=1}^{k} \frac{\mathbf{u}_{i}^{T} \mathbf{y}}{\lambda_{i}} \mathbf{v}_{i}+\sum_{i=1}^{N} \alpha_{i} \mathbf{E} \mathbf{v}_{i}, \\
\hat{\mathbf{y}}=\mathbf{E} \hat{\mathbf{x}}=\sum_{i=1}^{k} \mathbf{u}_{i}^{T} \mathbf{y} \mathbf{u}^{T}, \\
\hat{\mathbf{n}}=\mathbf{E} \hat{\mathbf{x}}=\sum_{i=1}^{M} \mathbf{u}_{i}^{T} \mathbf{y} \mathbf{u}^{T} .
\end{array}
$$

The indeterminate $\alpha_{i}^{\prime}$ 's are arbitrary and represent structures in the solution for which the equations provide no information. Because we can not find their value, it is customary to seek the simplest solution; this is done by setting $\alpha_{i}=0, i>k$, so

$$
\hat{\mathbf{x}}=\sum_{i=1}^{k} \frac{\mathbf{u}_{i}^{T} \mathbf{y}}{\lambda_{i}} \mathbf{v}_{i}
$$

The $\mathbf{v}_{i}$ corresponding to $\lambda_{i}=0$ are called the solution null space.

SVD is also useful when the matrix is ill-conditioned, when, despite $\lambda_{i} \neq 0$ is satisfied, there are some structures of the solution that can not be estimated with sufficient reliability because some $\lambda_{i}$ are too "small". In this case, the terms corresponding to the small $\lambda_{i}$ are dumped into the nullspace, now called the effective nullspace, by setting those small $\lambda_{i}=0$. The solution is then truncated at $k^{\prime}<k<M$,

$$
\hat{\mathbf{x}}=\sum_{i=1}^{k^{\prime}} \frac{\mathbf{u}_{i}^{T} \mathbf{y}}{\lambda_{i}} \mathbf{v}_{i}+\sum_{i=k^{\prime}+1}^{k} \alpha_{i} \mathbf{E v}_{i}+\sum_{i=k+1}^{N} \alpha_{i} \mathbf{E v}_{i}
$$

The truncation reduces the uncertainty but increases the nullspace contribution. It also offers a simple method for controlling the ratio between the solution and the residual norms. There are no rules to determine objectively how small a $\lambda_{i}$ needs to be in order to be placed in the null space.

\section{Damped least squares.}

SVD deals with singular and ill conditioned matrices by finding the spanning set for the range of $\mathbf{E}$. Damped least squares, on the other hand, suppresses the null space by adding a positive value to the diagonal of a singular or ill conditioned matrix, 
making it have an inverse. The generalized method consists of replacing the problem $\mathbf{E x} \simeq \mathbf{y}$ by appending additional rows and obtain,

$$
\left[\begin{array}{l}
\mathrm{E} \\
\mathbf{F}
\end{array}\right] \mathrm{x} \simeq\left[\begin{array}{l}
\mathbf{y} \\
\mathrm{g}
\end{array}\right],
$$

where $\mathbf{F}$ is an $l \times n$ matrix and $\mathbf{g}$ is an $l$-dimensional vector.

By appending these rows we gain control over the value of $\mathbf{x}$. Suppose it is desired that $\mathbf{x}$ be close to a known vector $\hat{\mathbf{x}}$; this can be done by setting $\mathbf{F}=\mathbf{I}$ and $\mathbf{g}=\hat{\mathbf{x}}$ in Equation A.22. The tapered least squares case is obtained by setting $\mathbf{g}=\hat{\mathbf{x}}=0$, which expresses the preference for $\|\mathbf{x}\|$ to be small. The intensity of this preference is indicated by a scaling parameter, $\alpha^{2}$, incorporated into the definition of $\mathbf{F}$ and $\mathbf{g}$ as,

$$
\begin{aligned}
& \mathbf{F}=\alpha^{2} \mathbf{I} \\
& \mathbf{g}=\alpha^{2} \hat{\mathbf{x}} .
\end{aligned}
$$

The parameter $\alpha^{2}$ can be regarded as the inverse of the estimate of the size of the uncertainty in $\hat{\mathbf{x}}$. Setting $\alpha^{2}$ to a high value causes the solution to be closer to $\hat{\mathbf{x}}$. The parameter $\alpha^{2}$ is introduced so the cost function that needs to me minimized becomes:

$$
J=(\mathbf{E x}-\mathbf{y})^{T}(\mathbf{E x}-\mathbf{y})+\alpha^{2} \mathbf{x}^{T} \mathbf{x}
$$

The challenge in this method is to decide the value of $\alpha^{2}$ sometimes called damping or ridge. One way for deciding on the value of $\alpha^{2}$ is by means of the Marquardt Diagram, a plot of the magnitude of the noise, $|\mathbf{n}|$, against the magnitude of the parameters, $|\mathbf{x}|$ (figure A-1). $|\mathbf{x}|$ is inversely proportional to $\alpha^{2}$, while $|\mathbf{n}|$ is directly proportional. The $\alpha$ value can be chosen such that minimizes both $|\mathbf{n}|$ and $|\mathbf{x}|$, at the point where, increasing $\alpha^{2}$ from $\alpha^{2} \rightarrow 0,|\mathbf{x}|$ decreases while $|\mathbf{n}|$ varies little.

Nothing prevents from having different uncertainties for each component of $\hat{\mathbf{x}}$. This seems to make sense in the orbit error case as the ocean is not homogeneous and 


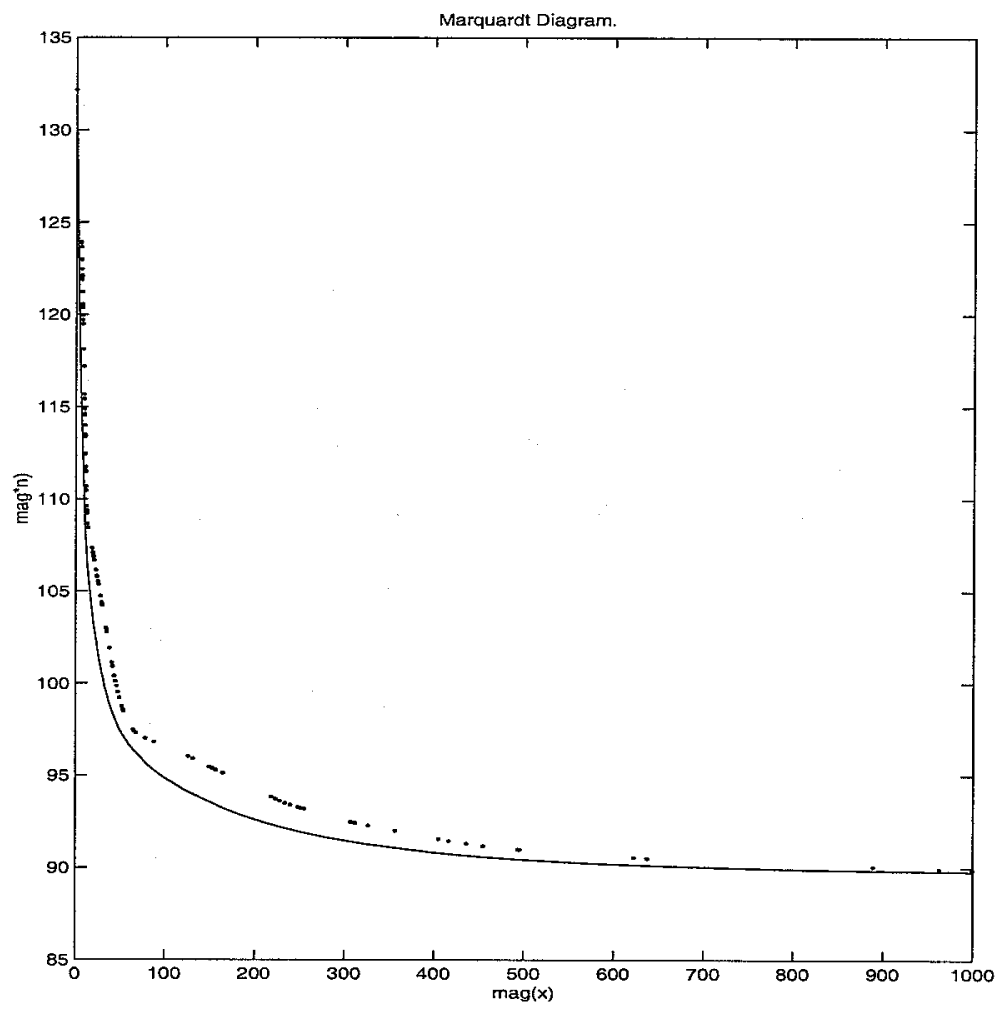

Figure A-1: Marquardt diagram for Tapered Least Squares (solid line) and Singular Value Decomposition (dots). 
the time lag between transects at crossovers varies among them. It seems sensible to expect the crossover difference not due to the orbit error to be proportional to the time lag between transects of the same cross-over and inversely proportional to the time decorrelation scale of oceanographic process (i.e. decorrelation time scale close to the Gulf Stream is shorter than away from it). A matrix $\mathbf{S}$ can be defined to be used instead of $\alpha^{2}$ so that the cost function to be minimized becomes:

$$
J=(\mathbf{E x}-\mathbf{y})^{T}(\mathbf{E x}-\mathbf{y})+\mathbf{S}^{-1} \mathbf{x}^{T} \mathbf{x},
$$

whose solution is,

$$
\tilde{\mathbf{x}}=\left(\mathbf{E}^{T} \mathbf{E}+\mathbf{S}^{-1}\right)^{-1} \mathbf{E}^{T} \mathbf{y} .
$$

The particular case in which $\mathbf{S}^{-1}=\alpha^{2} \mathbf{I}$ corresponds to the tapered least squares. In this case, when enough information regarding the solution is at hand, such that its second moment, $\mathbf{R}_{x x}$, is known, then $S=\mathbf{R}_{x x}$ can be chosen.

The value of the subrank is obtained with the Marquard Diagram for both, SVD and TLS. The Marquardt Diagram is formed by varying the subrank for SVD and the taperer for TLS. The resulting magnitude of the noise, $|\mathbf{n}|$, is plotted against the magnitude of the solution, $|\mathbf{x}|$. The point that minimizes both, $|\mathbf{n}|$ and $|\mathbf{x}|$ is selected. In order to do this automatically in an objective manner, I use the slope of the diagram as a criterion for selecting such point. The TLS curve can be made as continuous as needed, there is significant control on the value of $\alpha^{2}$, making the use of the slope is possible. The SVD curve is not continuous and its shape is predetermined by the value of the singular values; in Figure A-1, the points represent the SVD points while the continuous line represents the TLS curve. The slope criterion is not feasible. I chose to select the point on the TLS curve where the gradient is closest to -0.001 , and find the nearest point of the SVD and its corresponding subrank. The process is repeated for each time realization. 


\section{Bibliography}

[1] Bendat, J.S. and A.G. Piersol. 1986. Random Data: Analysis and Measurement Procedures. 2nd edition. Wiley-Interscience, New York. 566 pp.

[2] Batchelor, G.K. 1953. The Theory of Homogeneous Turbulence. Cambridge University Press. 197 pp.

[3] Bower, A.S., and N.G. Hogg. 1992. Evidence for barotropic wave radiation from the Gulf Stream. Jour. Phys. Oceanogr. 22, 42-61.

[4] Bretherton, F.P., R.E. Davis, C.B. Fandry. 1976. A technique for objective analysis and design of oceanographic experiments applied to MODE-73. Deep Sea Res. 23, 559-782.

[5] Bryan, K. 1969. A numerical method for the study of the circulation of the world ocean. Jour. Compt. Phys. 4, 347-376.

[6] Chelton et al. 2001

[7] Cheney, R. E., and J. G. Marsh. 1983 Global mesoscale variability from repeat tracks of Seasat altimeter data. Jour. Geophys. Res. 88, 4343-4354.

[8] Cutting, E., G. H. Born, and J. C. Frautnick. 1978. Orbit analysis for SEASATA. Jour. Astronaut. Sci. 26, 315-342.

[9] Duckowicz, J.K. and R.D. Smith. 1994. Implicit free surface method for the Bryan-Cox-Semtner ocean model. Jour. Geophys. Res. 99, 7991-8014. 
[10] Fu, L.L. and A. Cazenave, eds. 2001. Satellite Altimetry and Earth Sciences: A Handbook of Techniques and Applications. Academic Press, Inc., Boston. 463 pp.

[11] Fu, L.-L., D.B. Chelton. 1985. Observing large-scale temporal variability of ocean currents by satellite altimetry: with application to the Antarctic Circumpolar Current. Jour. Geophys. Res. 90, 4721-4789.

[12] Fu, L.-L., E.J. Christensen, C.A. Yamarone, M. Lefebvre, Y. Menard, M. Dorrer and P. Escudier. 1994. TOPEX/POSEIDON mission overview. Jour. Geophys. Res. 99, C12, 24369-24381.

[13] Gill, A.E. 1982. Atmosphere-Ocean Dynamics. Academic Press, Inc., Boston. 662 pp.

[14] Glazman, R.E., A. Fabrikant, and A. Greysukh. 1996. Statistics of spatialtemporal variations of sea surface height based on Topex altimeter measurements. Int. J. Remote Sensing. 17, 2647-2666.

[15] Gordon, A. L., K.-I. Horai, and M. Donn. 1983. Southern Hemisphere western boundary current variability revealed by GEOS 3 altimeter. Jour. Geophys. Res. 88 (C1) 755-762.

[16] Hogg, N.G. 1983. A note on the deep circulation of the western North Atlantic: its nature and causes. Deep Sea Res. 30, 945-961.

[17] Hogg, N.G. 1988. Stochastic wave radiation by the Gulf Stream. Jour. Phys. Oceanogr. 18, 1687-1701.

[18] Hogg, N.G. 1993. Towards the parametrization of the eddy field near the Gulf Stream. Deep Sea Res. 40, 2359-2376.

[19] Jayne, S.R., N.G. Hogg, and P. Malanotte-Rizzoli. 1996. Recirculation gyres forced by a beta-plane jet. Jour. Phys. Oceanogr. 26, 492-504. 
[20] King, C., D. Stammer and C. Wunsch. 1994. The CMPO/MIT TOPEX/POSEIDON altimetric data set. Center for Global Change Science, Massachusetts Institute of Technology. Rep. 30, $33 \mathrm{pp}$.

[21] Kundu, P.K. 1990. Fluid Mechanics. Academic Press, Inc., Boston. 638 pp.

[22] Lawson, C. L., and R. J. Hanson. 1974. Solving Least Square Problems. PrenticeHall, Inc. New Jersay. 340 pp.

[23] Levitus, S. 1982. Climatological Atlas of the World Ocean. NOAA Prof. Paper No. 13. U.S. Government Printing Office, Washington, D.C. 173 pp.

[24] Malanotte-Rizzoli, P., N.G. Hogg, and R.E. Young. 1995. Stochastic wave radiation by the Gulf Stream: numerical experiments. Deep Sea Res. 42, 389-423.

[25] Marsh, J. G., and R. G. Williamson. 1980. Precision orbit analysis in support of the SEASAT altimeter experiment. Jour. Astronaut. Sci. 28, 345-396.

[26] Marshall, J. A., N. P. Zelensky, S. M. Klosko, D. S. Chinn, S. B. Lutchke, K. E. Rchlin, and R. G. Williamson. 1995. The temporal and spatial charachteristics of TOPEX/POSEIDON radial orbit error. Jour. Geophys. Res. 100 (C12) 2533125352.

[27] Morrow, R., R. Coleman, J. Church and D. Chelton. 1994. Surface eddy momentum flux and velocity variances in the southern ocean from Geosat altimetry. Jour. Phys. Oceanogr. 24, 2050-2071.

[28] Nishida, H., and W.B. White. 1982. Horizontal eddy fluxes of momentum and kinetic energy in teh near-surface of the Kuroshio Extension. Jour. Phys. Oceanogr. 12, 160-170.

[29] Parke, M. E $\dot{i}$, R. H. Stewart, and D. L. Farless. 1987. On the choice of orbits for an altimetric satellite to study ocean circulation and tides. Jour. Geophys. Res. 92 (c11), pp.11,693-11,707. 
[30] Pedlosky, J. 1987. Geophysical Fluid Dynamics. 2nd edition. Springer-Verlag, New York. 710 pp.

[31] Peterson, R.G. 1992. The boundary currents in the western Argentine Basin. Deep Sea Res. 39, 623-644.

[32] Preisendorfer, R. W. 1988. Principal COmponent Analysis in Meteorology and Oceanography. Elsevier, 425 pp.

[33] Priestly, M.B. 1981. Spectral Analysis and Time Series. Academic Press, Inc., San Diego. 890 pp.

[34] Rhines, P.B. 1977. The dynamics of unsteady currents. In The Sea: Ideas and Observations on Progress in the Study of the Seas. 6: Marine Modeling. pp. 189318. I.N. McCave, J.J. O'Brien, and J.H. Steele. (eds) Wiley-Interscience, New York.

[35] Richardson, P.L. 1983. Eddy kinetic energy in the North Atlantic from surface drifters. Jour. Geophys. Res. 88, 4355-4367.

[36] Saunders, P.M., and B.A. King. 1995. Bottom currents derived from a shipborne ADCP on WOCE A11 in the South Atlantic. Jour. Phys. Oceanogr. 25, 329-347.

[37] Schmitz, W. J.Jr. 1980. Weakly depth-dependent segments of the North Atlantic circulation, Jour. Marine res. 38, 111-135.

[38] Schmitz, W. J.Jr. 1982. A comparison of the midd-latutude eddy fields in western North Atlantic and the Norht Pacific Ocean. Jour. Phys. Oceanogr. 12, 208-210.

[39] Sokal, R.R. and F.J. Rohlf. 1981. Biometry: The Principles and Practice of Statistics in Biological Research. 2nd edition. Freeman, San Francisco. 859 pp.

[40] Smith, R.D., M.E. Maltrud, F.O. Bryan, and M.W. Hecht. 2000. Numerical simulation of the North Atlantic Ocean at $1 / 10^{\circ}$. Jour. Phys. Oceanogr. 30, 1532-1561. 
[41] Stammer, D., and C. W. Böning. 1992. mesoscale variability in the Atlantic Oean from geosat altimetry and WOCE high-resolution numerical modelling. Jour. Phys. Oceanogr. 22, 732-752.

[42] Stammer, D., and C. W. Böning. 1996. Generation and distribution of mesoscale eddies in the North Atlantic. In The Warmwatesphere of the North Atlantic Ocean. pp, 159-198. W. Krauss (ed). Gebrüder Borntraeger. Berlin, Stuttgard.

[43] Stammer, D. 1997. Global charachteristics of ocean variability estimated from regional TOPEX/POSEIDON altimetric measurements. Jour. Phys. Oceanogr. 27, 1743-1769.

[44] Stammer, D. and C. Wunsch. 2000. De-aliasing of global high frequency barotropic motions in altimeter observations. Geophys. Res. Let. 27(8), 11751178.

[45] Tai, C.-K. 1988. Geosat crossover analysis in the tropical Pacific. 1. Conatrained sinusoidal crossover adjustment. Jour. Geophys. Res. 93 10621-10628.

[46] Tai, C.-K. 1989. Accuracy assesment of widely used orbit error approximation in satellite altimetry. Jour. Atmos. Ocean. Tech. 6, 147-150.

[47] Tai, C.-K. 1991. How to observe the Gyre to Global-Scale variability in satellite altimetry: signal attenuation by orbit error removal. Jour. Atmos. Ocean. Tech. 8, 271-288.

[48] Tai, C.-K., and L.L. Fu. 1986. On crossover adjustment in satellite altimetry and its oceanographic implications. Jour. Geophys. Res. 91, (C2), 2549-2554.

[49] Tai, C.-K., and J. M. Kuhn. 1994. On reducing the large-scale time dependent errors in satellite altimetry while preserving the ocean signal: orbit and tide error reduction for TOPEX/POSEIDON. NOAA Tech. Memo., NOS EOS 9, 29 pp. 
[50] Tai, C.-K., and J. M. Kuhn. 1995. Orbit and tide error reduction for the first two years of TOPEX/POSEIDON data. Jour. Geophys. Res. 100, (C12), 2535325363.

[51] Tai, C.-K., and C. A. Wagner. 1994. Along-track spectra of the ocean seasonal cycle and their attenuation by various orbit error removal schemes in satellite altimetry. NOAA Tech. Memo., NOS EOS 10, 16 pp.

[52] Tai, C.K., and W.B. White. 1990. Eddy variability in the Kuroshio Extension as revealed by Geosat altimetry: energy propagation away from the jet, Reynold stress and seasonal cycle. Jour. Phys. Oceanogr. 20, 1761-1777.

[53]. Webster, F. 1961. The effect of meanders on the kinetic energy balance of the Gulf Stream. Tellus. 13, 392-401.

[54] Wunsch, C. 1981. Low Frequeney variability of the Sea. In Evolution of Physical Oceanography. Scientific Surveys in Honor of Henry Stommel, pp. 6-41, B. A. Warren \& C. Wunsch (Eds.) The MIT Press, Cambridge, U.S.A.

[55] Wunsch, C. 1996. The Ocean Circulation Inverse Problem. Cambridge University Press, New York. 442 pp.

[56] Wunsch, C. 1997. The vertical partition of oceanic horizontal kinetic energy. Jour. Phys. Oceanogr. 27, 1770-1794.

[57] Wunsch, C., and D. Stammer. 1995. The global frequency-wavenumber spectrum of oceanic variability estimated from TOPEX/POSEIDON altimeter measurements. Jour. Geophys. Res. 100, 24895-24910. 